\title{
Quantitive Photoemission Spectroscopy of Hydrogen Bonded Systems
}

\author{
Dissertation \\ zur Erlangung des Doktorgrades \\ der Mathematisch-Naturwissenschaftlichen Fakultäten \\ der Georg-August-Universität zu Göttingen
}

vorgelegt von

\section{Yaxing Liu}

aus Qufu, VR China

Göttingen 2010 
Referent:

Prof. Dr. Bernd Abel

Korreferent:
Prof. Dr. Peter Botschwina

Tag der mündlichen Prüfung: 21.Juli.2010 
No one really understands water. It's embarrassing to admit it, but the stuff that covers two-thirds of our planet is still a mystery.

Phillip Ball, Water - an enduring mystery 



\section{Danksagung}

Viele Personen haben zum Entstehen meiner Doktorarbeit beigetragen. Sie sollen an dieser Stelle im einzelnen genannt werden.

Herrn Prof. Dr. Jürgen Troe danke ich für die freundliche Aufnahme in seiner Abteilung und die förderlichen Arbeitsbedingungen.

Meinem Chef, Herrn Prof. Dr. Bernd Abel gilt mein Dank für die Hilfsbereitschaft bei allen erdenklichen Problemen und die engagierte Unterstützung.

Herrn Prof. Dr. Peter Botschwina danke ich für die freundliche Übernahme des Korreferates.

Meinen Kollegen Katrin Siefermann, Euvgeny Lugovoy und Oliver Link gebührt mein herzlicher Dank für die tolle Laborgemeinschaft, fruchtbare Diskussion und gegenseitige Unterstützung in allen stressigen Situationen. Insbesondere danke ich Katrin und Peter Lohse, meinem Büromitbewohner, für das fleißige Korrekturlesen.

Herrn Jun.-Prof. Dr. Klaus Ropers und seinem Mitarbeiter Murat Sivis danke ich für die harmonische Kooperation beim Nanostruktur-Projekt.

Herrn Dr. Manfred Faubel danke ich für die zahlreichen Diskussionen und Anregungen rund um das Thema Photoelektronenspektroskopie.

Herrn Prof. Dr. Udo Buck danke ich für die Hilfestellung und Diskussion beim Thema Cluster-Erzeugung durch adiabatische Expansion.

Der Arbeitsgruppe Prof. Dr. Martin Suhm danke ich für die Bereitstellung der Düse für die Cluster-Erzeugung. Ohne diese wären die Cluster-Messungen nicht möglich gewesen.

Allen Mitgliedern der Abteilung Troe, besonders den restlichen Mitarbeitern der Abel-Gruppe danke ich für hervorragende Zusammenarbeit und die Begleitung in den schönen Jahren in der Gruppe. 
Bei Herrn Werner Noack und allen Mitarbeitern der Werkstätten des Instituts für Physikalische Chemie bedanke ich mich für die schnelle und kompetente Erledigung aller meiner Aufträge.

Einen herzlichen Dank spreche ich meiner großen Familie und allen den Menschen aus, die während der schwierigsten Phase meines Lebens stets zu mir standen und mich in allen Situationen ausdauernd unterstützten.

Ich danke Dir Alexander von ganzem Herzen, dass Du immer für mich da bist und mir zeigst, wie schön das Leben sein kann. Bald kann die räumliche Entfernung uns nicht mehr trennen. Darauf freue ich mich! 


\section{Contents}

$\begin{array}{ll}\text { Abstract } & 1\end{array}$

\begin{tabular}{lll}
\hline 1 & Introduction & 3
\end{tabular}

\begin{tabular}{|lll}
2 & UV Photoelectron Spectroscopy & 7
\end{tabular}

2.1 Photoionization . . . . . . . . . . . . . . . . . . . . . . . . . . . . 7

2.2 Information obtained from PE Spectra . . . . . . . . . . . . . . 8

2.3 Experimental aspects . . . . . . . . . . . . . . . . . . . 10

$2.3 .1 \quad$ Ionizing photon sources . . . . . . . . . . . . . . . . . 10

$2.3 .2 \quad$ Analyzing electron energy . . . . . . . . . . . . . . . . . 12

2.3 .3 Sample handling . . . . . . . . . . . . . . . . . . 13

2.4 Theory of photoemission . . . . . . . . . . . . . . . . 13

3 High Harmonic Generation 19

3.1 Introduction . . . . . . . . . . . . . . . . . . . . . 19

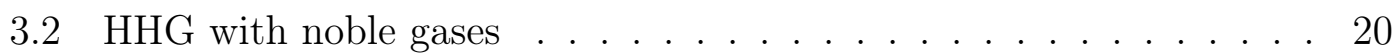

3.2 .1 The three-step model . . . . . . . . . . . . . . . . . . . 20

3.2 .2 The EUV source . . . . . . . . . . . . . . . . . . . . . . . . 24

3.2 .3 Characteristics of high harmonics . . . . . . . . . . . . . . 26

3.3 HHG with nano-structures . . . . . . . . . . . . . . . . . . . . . . . 27

3.3 .1 Construction of nano-structures . . . . . . . . . . . . . . . 28

3.3 .2 8fs-Oscillator system . . . . . . . . . . . . . . . . . . . 29

3.3.3 Experimental setup . . . . . . . . . . . . . . . 30

3.3 .4 Preliminary results and outlook . . . . . . . . . . . . . . 31

$\begin{array}{lll}4 & \text { Experimental approach } & 37\end{array}$

4.1 The laser system . . . . . . . . . . . . . . . . . . . . . . . . . . 38 
4.2 Light polarization tuning . . . . . . . . . . . . . . . . . . . . . 41

4.3 The vacuum apparatus . . . . . . . . . . . . . . . . . . . . . . . 42

4.4 Liquid micro-jet . . . . . . . . . . . . . . . . . . . . . . . . . . . 44

4.5 Cluster source $\ldots \ldots \ldots$. . . . . . . . . . . . . . . . . . . 46

4.6 TOF-spectrometer $\ldots \ldots \ldots \ldots \ldots$

5 Angular distribution of photoemission from water 51

5.1 Introduction . . . . . . . . . . . . . . . . . 51

5.2 Angle-dependent photoelectron spectra . . . . . . . . . . . . . . . 60

$5.2 .1 \quad$ PE spectra from liquid water . . . . . . . . . . . . . . 60

5.2 .2 PE spectra from small water clusters . . . . . . . . . . . 66

5.3 Determination of the anisotropy parameter $\beta$. . . . . . . . . . . 71

5.4 Results and discussion . . . . . . . . . . . . . . . . 76

6 Angular distribution of photoemission from methanol 93

6.1 Introduction . . . . . . . . . . . . . . . . . . . . . 93

6.2 Angle-dependent photoelectron spectra . . . . . . . . . . . . . . . . 95

6.3 Results and discussion . . . . . . . . . . . . . . . . . . . . . . . . . . . . . 99

\begin{tabular}{ll}
\hline List of Figures & 105
\end{tabular}

\begin{tabular}{ll}
\hline List of Tables & 107
\end{tabular}

\begin{tabular}{lr}
\hline Bibliography & 109
\end{tabular} 


\section{Abstract}

Water, the most crucial substance on our planet, is characterized by its hydrogen bond network. Although numerous investigations have been carried out to study the nature of the hydrogen bond, the electronic structure of hydrogen bonded water molecules on the level of molecular orbitals has not yet been revealed completely. In particular, no angle-dependent measurement of photoemission spectra has been performed in order to determine the anisotropy parameters for the valence orbitals of water molecules in liquid phase, so far. The spatial distribution of the photoelectrons ejected from a particular orbital is anisotropic and can be expressed in terms of a parameter $\beta$. Besides its dependence on ionizing photon energy, the anisotropy parameter $\beta$ is determined by the topology of the corresponding orbital. As a consequence of formation of hydrogen bonds, deformation of involved orbitals takes place, which results in change of $\beta$ value for each orbital.

In the present work, an angle-dependent photoelectron spectroscopy experiment is performed in combination with a table-top EUV radiation source based on high harmonic generation (HHG). For the first time, the $\beta$ values $(\mathrm{h} \nu=38.7 \mathrm{eV})$ for the $1 b_{1}, 3 a_{1}$ and $1 b_{2}$ orbitals in liquid water are determined to be $0.8,0.7$ and 0.6 respectively, in contrast to the values $1.4,1.1$ and 0.7 in the gas phase. The smaller $\beta$ values related to the liquid phase, result from delocalization of the orbitals due to the significant interactions between hydrogen bonded molecules in liquid water. Moreover, the change of the photoemission anisotropy is different for the three orbitals: the $1 b_{1}$ and $3 a_{1}$ orbitals exhibit significant decrease of $\beta$ value while the $1 b_{2}$ shows only slight variation. This indicates that $1 b_{1}$ and $3 a_{1}$ orbitals have the most contribution to hydrogen bonding.

Also the $\beta$ parameter is determined for the $1 b_{1}$ orbital in small-sized clusters $(\langle n\rangle=5)$, which amounts to 1.0. The small difference between the photoemission anisotropy for liquid water and small clusters is assumed to be an indication for the 
major influence of the first coordination shell on the local electronic structure in the hydrogen-bonded networks. Besides, it might be an evidence that the average coordination number is between 2 and 3 in liquid water.

In order to reveal the nature of hydrogen bonds in systems comparable to water, an analog study is carried out on methanol. The comparative study on angular photoemission of methanol indicates the distinctly strong hydrogen bonding in water as compared to methanol.

As an addition to the general topic of the present work, the development of a novel method for high harmonic generation is presented. Such modern EUV sources can advantageously be driven by Ti:Sapphire oscillators with low pulse intensity and are more compact than the existing HHG sources with rare gases as nonlinear media. Therefor, nano-structures with bow-tie shaped elements are applied to induce local field enhancement of the initial laser field. 


\section{Chapter 1}

\section{Introduction}

Water is the most essential substance for all living organisms on our planet and exists concurrently in different aggregate states in the nature. Particularly, liquid water is very crucial for numerous important processes in chemistry, biology, environmental science and planetary physics. Therefore, water has been the most extensively investigated compound ever. However, water still remains an enduring mystery [1].

Water is especially characterized by its unique properties, such as the density anomaly at $4^{\circ} \mathrm{C}$, the strong polarizability and significant increase of dipole moment in the liquid phase [2]. Those distinctive characteristics are associated with the most notable intermolecular interaction - hydrogen bonding. In all the dense aggregate states of water, hydrogen bonds are formed in networks with different mechanisms. In the liquid phase, the hydrogen bond network is disordered and dynamic. Hydrogen bonds break and reform frequently due to the atomic motions. In contrast, the hydrogen bond arrangement in crystalline ice is fixed and structured. In cold clusters, the situation is different again. It is still unclear whether the water clusters are liquid-like or more similar to crystalline ice. Also the contributions to hydrogen bonding are disputed. Generally accepted, electrostatic attraction, polarization and dispersion are the essential components of this attractive interaction. However, another supposed contribution is gaining more and more popularity - charge transfer mechanism which is also described as partial covalency of hydrogen bond [3-6].

An especially active field within the numerous experimental and theoretical investigations of water is the study of electronic structure and the influence of hydro- 
gen bonding on it. For this purpose, valence photoelectron spectroscopy [6-13], X-ray emission spectroscopy [14-18] and X-ray absorption spectroscopy [19-21] have been applied to offer many important electronic informations about water in different hydrogen bonded aggregate states. However, the previous experimental investigations on the electronic structure of water were not able to directly examine the electron density distributions of hydrogen bonded water molecules. Only photoelectron-spectral variations relative to the spectra of free water molecules could be observed. These variations were interpreted as indications for a broad conduction band in the condensed phases or evolution of the electronic structure of valence orbitals and no quantitative change of the electron density distribution in hydrogen bonded water molecules could be concluded by those studies.

In this work, we investigate the angular distribution of photoemission from water and methanol - as a comparative system to water - in different aggregate states: gas, small clusters and liquid. The angular dependence of photoemission contains a vast amount of information which is highly useful in elucidating the electronic structure of atoms and molecules in different phases. The anisotropy parameter $\beta$ expresses the spatial distribution of photoelectrons from a certain orbital. Its value depends on the nature of the orbital and is very sensitive to electronic changes due to intermolecular interactions such as hydrogen bonding. Consequently, we probe these changes and our results can offer valuable clues to the understanding of hydrogen bonded systems.

Till now no experimental determination of the photoemission anisotropy parameter has been reported for molecular orbitals of liquid water or methanol. This absence lies in the challenge to design a suitable experimental apparatus. We were able to construct a profitable experiment to measure angle-dependent photoelectron spectra employing an EUV radiation source based on high harmonic generation. Via this approach we are able to easily adjust the polarization of the ionizing radiation by tuning of the polarization vector of the HHG driving fundamental laser light. In this way, we can analyze the photoelectrons at different emission angles between polarization vector of incident ionizing radiation and emission direction and can finally determine the desired value of $\beta$. Further our experimental setup is flexible regarding the measurement of samples in different 
aggregate states. Liquid micro-jet and a home-designed cluster source are available as alternative options for the sample handling.

The structure of the present work will be outlined in the following:

In chapter 2, basic principles of the UV Photoelectron Spectroscopy will be introduced. In particular, the theory of angular distribution of photoemission will be explained in detail, which serves as the theoretical basis of this study.

In chapter 3 , the employed EUV radiation source based on high harmonic generation will be presented including fundamental principles of the related nonlinear conversion process. In the last part of this chapter, the development of a novel HHG method will be demonstrated. The goal of such a project consists in inventing EUV sources which can be driven by Ti:Sapphire oscillators with low pulse intensity. Therefor, nano-structures with bow-tie shaped elements are applied to induce local field enhancement of the initial laser field.

Further details about the experimental setup are demonstrated in chapter 4 . The Ti:sapphire laser system, polarization tuning of the ionizing radiation, the liquid micro-jet technique and the cluster source are the highlights of this chapter.

The angular distributions of photoemission from liquid water and small-sized clusters will be presented in chapter 5. After that, the approach to determine the anisotropy parameters of valence orbitals will be described in detail. An intensive discussion about the relevance of the obtained results follows the presentation of the angular measurements of the photoelectron spectra.

In order to reveal the nature of hydrogen bonds in systems comparable to water, an analog study is carried out on methanol, which will be presented in chapter 6 . The angle-dependent photoemission study on methanol supplements the understanding of hydrogen bondings of different kinds, within the electronic picture of molecular orbitals. A comparison of the results from the angular photoemission studies on water and methanol can shed light onto the nature of hydrogen bonds on the molecular level of the examined systems. 



\section{Chapter 2}

\section{UV Photoelectron Spectroscopy}

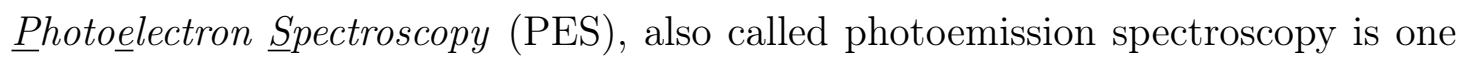
of the most established spectroscopic methods and based on the phenomenon of photoemission, which was observed by Hertz in 1887 [22]. It was Einstein who was able to explain this observation in 1905 by invoking the quantum nature of light [23]. Since then many experiments were performed with the same principle but various experimental approaches, and there has been intensive activity in the general field of PES. Ultraviolet photoelectron spectroscopy (UPS) is one fundamental and the most important branch of PES [24].

\subsection{Photoionization}

The wavefunction of an electron is called an orbital and its square is the probability distribution around the nucleus or nuclei of an atom or molecule. Within the orbital concept, the behavior of electrons can be conveniently illustrated. Depending on the nature of the related orbitals, there are two kinds of electrons: core and valence electrons. The former are close to the nucleus and in the inner orbitals of atoms. The valence electrons are further away from the nucleus and directly involved in chemical bonds between atoms, in contrast to the core electrons. Photoelectron spectroscopy is a powerful tool to examine the electronic structure of atoms or molecules, which makes use of photoionization process. Induced by the absorption of a photon with enough high energy, an electron can be promoted into vacuum level and escapes the binding forces of an atom or a molecule. In this case, photoionization takes place. 
Here is a simple case of photoionization: an atom with $N$ electrons emits one electron by absorbing a photon $h \nu$ :

$$
\mathrm{A}_{N}+h \nu \rightarrow \mathrm{A}_{N-1}^{+}+e^{-}
$$

The minimum energy required for this process is the ionization energy of an electron in that particular orbital. Any photon with an energy higher than the ionization limit can bring about photoionization. This does not break the resonance condition because the electron is formally excited into the continuum of free states and can take any excess energy in the form of electron kinetic energy.

Differing from conventional electronic absorptions, electron energies are measured rather than photon energies. For the upper case, a part of the incoming photon energy $(h \nu)$ is used to ionize the atom. The remainder is partitioned between the atomic cation and the electron kinetic energy and so, from the conservation of energy results:

$$
h \nu=I E_{i}+T_{\mathrm{A}_{N-1}^{+}}+T_{e} .
$$

Here, $I E_{i}$ is the ionization energy of an electron in orbital $\mathrm{i}$ and $T_{\mathrm{A}_{N-1}^{+}}$and $T_{e}$ are the kinetic energies of cation and electron. Since an electron is very much lighter than an atomic nucleus, $T_{\mathrm{A}_{N-1}^{+}}$usually can be neglected and most of the kinetic energy will be taken away by the electron. If the photon energy is fixed, a spectrum can be obtained by measuring the electron current as a function of electron kinetic energy or as a function of orbital ionization energy using

$$
I E_{i}=h \nu-T_{e} .
$$

This is the basic idea of photoelectron spectroscopy.

\subsection{Information obtained from PE Spectra}

An extraordinary range of information can be deduced from photoelectron spectra. A typical UPS spectrum is shown in Fig.2.1. First and most obviously, information of orbital energies in atoms or molecules can be obtained assuming Koopmans' 
Theorem (oder sudden approximation). This considers the nucleus and the rest $(N-1)$ electrons in $\mathrm{A}_{N-1}^{+}$as rigid during an one photon ionization of atom $\mathrm{A}$. The wave functions of nucleus and remaining electrons do not vary and no electronic relaxation occurs. This postulates equality of the negative of the ionization energy and one-electron orbital energy. This approximation results in errors of about $1-2 \mathrm{eV}$ [24], since it neglects contributions of ultrafast electronic relaxation during the ionization process. However, in most cases Koopmans' Theorem is very useful, because it allows to distinguish one orbital from another.

There is also another approach called adiabatic approximation. In contrast to the sudden approximation, the remaining electrons of the ionized molecule relax during the ionization time, because the ejected electron leaves the system so slowly that other electrons can adjust their effective potential in a self-consistent way. The kinetic energy of the ejected electron is the energy difference between the photon energy and the ionization potential which correlates with both the energy of the neutral molecule and that of the relaxed ion. Accordingly, binding energies cal-

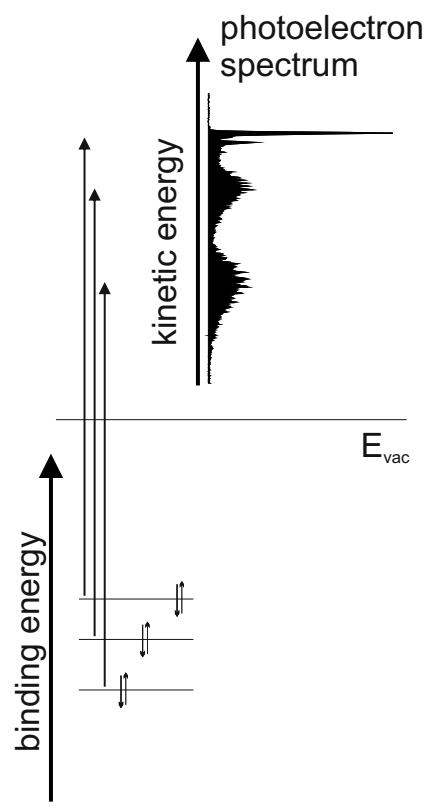

Figure 2.1: Sketch illustrating the mechanism behind PES. After ionizing electrons, their kinetic energy and occurrence is detected which results in an photoelectron spectrum. Here, a typical UPS spectrum of free water molecule is shown. 
culated with the adiabatic approximation are always smaller than those obtained with the sudden approximation.

However, PES can provide more than just details of orbital energies. Vibrational structure can be resolved very often, particularly for molecules in the gas phase. This leads directly to vibrational frequencies. Qualitative and sometimes even quantitative information on the structure of the molecule can be obtained in this way [25]. The Franck-Condon factors can also be deduced from the relative electron fluxes for the various vibrational states of the ion.

\subsection{Experimental aspects}

There are various available approaches to perform a PES experiment. A sketch of a basic PES setup is shown in Fig. 2.2. The different variations of PES experiments are not discussed in detail here. This section rather focuses on the three essential parts of PES: ionizing photon sources, electron analyzer, and samples. Ultra high vacuum equipment which is a further prerequisite for PES experiments will not be treated here in detail.

\subsection{1 lonizing photon sources}

The energy of the ionizing photon can be in the ultraviolet regime (5 to $100 \mathrm{eV}$, UPS), in the soft X-ray regime (100 to $1000 \mathrm{eV}$, SXPS) or in the X-ray regime (>1000 eV, XPS). With photon energies under $100 \mathrm{eV}$ valence electrons can be ejected while photoionization on core levels can only be observed with PES using X-ray sources. Because core level energies strongly depend on the chemical state

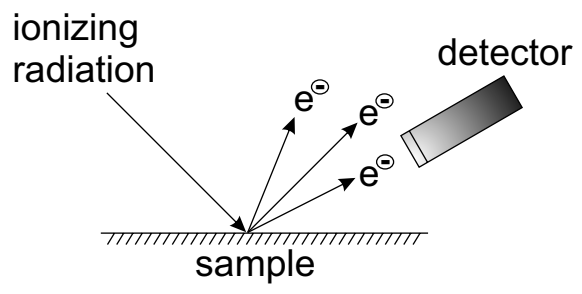

Figure 2.2: Illustration depicting the principle of PES experiments. 
of the sample, the spectroscopy of core levels can often be used for chemical analysis. The name ESCA (Electron Spectroscopy for Chemical Analysis) has been established for this technique devised by Siegbahn since 1969 [26].

The first ionization energies of the majority of molecules are larger than $9 \mathrm{eV}$ and fall in the vacuum ultraviolet region. It is difficult to generate monochromatic light in this region with sufficient intensities. In fact, this problem was not resolved until the early 1960s, when the noble gas resonance lamp sources were introduced.

One of the main instrumental advances of UPS during the 1970s is connected to the development and improvement of helium radiation sources. In VUV noble gas resonance lamps, two methods are used to promote noble gas atoms up to excited electronic states: the traditional method with a high voltage DC discharge between $\mathrm{Al}$ electrodes and a second method employing a microwave discharge. The latter is apparently necessary when very pure helium is used. After electronic excitement, the transition back to the ground state results in the emission of radiation. For helium, the principal emission line is at $21.218 \mathrm{eV}$ and arises from the transition ${ }^{1} \mathrm{P}\left(1 s^{1} 2 p^{1}\right) \rightarrow{ }^{1} \mathrm{~S}\left(1 s^{2}\right)$. This line is referred to as the HeI $\alpha$ line. Also a number of satellite lines and lines from transitions in $\mathrm{He}^{+}$, the HeII lines become apparent albeit with much weaker intensity.

Over the years, numerous important UPS experiments have been performed using synchrotron radiation from electron storage rings. To generate synchrotron radiation, a discharge of electrons is injected into a storage ring and confined to a circlular path by a series of magnets. The electrons are accelerated to nearly the speed of light and lose energy in the process in form of synchrotron radiation. The light can be extracted at various beamline stations and applied in different experiments. The essential benefits of synchrotron radiation for PES are a wide wavelength range $\left(10^{-10}-10^{-5} \mathrm{~m}\right)$, the high intensity and the plane polarization. With a suitable monochromater a specific photon energy can be selected.

Both of the radiation sources mentioned above are not suitable for generating pulses with subpicosecond duration. If one wants to construct a time resolved photoelectron spectroscopic experiment to investigate ultrafast dynamics, high coherent UV radiation obtained via high harmonic generation (HHG) may be the best alternative at the present time. We generate the ionizing radiation in 
our experiment also via HHG. Notice the details of high harmonic generation in Chap. 3 .

In the near future, a Free Electron Laser (FEL) will probably be the best choice to generate ultrashort XUV or X-ray pulses with high brilliance [27]. The main restrictions of a FEL are the required large amount of investments and immense storage area. Access to FELs is limited and only available for selected research groups.

\subsubsection{Analyzing electron energy}

There are two main analyser types to determine electron kinetic energies: retarding field and deflection analyzers. The former devices transmit only electrons which have energies higher than the retarding potential and is rarely used at the present time.

Deflection analyzers distinguish electrons by forcing them to follow different paths corresponding to their velocities. There are mainly three types of them: parallel plate analyzer, cylindrical mirror analyzer and hemispherical analyzer. The last one is used most prevalently. Its basic functional manner is as follows: two concentric hemispherical electrodes are charged to a potential with the same magnitude but opposite signs. Slits at the entrance and exit to the analyzer define the range of acceptable entrance and exit trajectories of the electrons. Only electrons with a certain energy, which is determined by the selected voltages on the hemispheres can traverse the analyzer and be detected by electron multipliers. The most important properties of such a photoelectron spectrometer are its resolution and sensitivity. A compromise must be taken, to find a trade-off between acceptable resolution and good sensitivity. The common resolution is in the $10-30 \mathrm{meV}$ range.

There is a special analyzer which is not very widely used for analyzing electron kinetic energies, the time-of-flight photoelectron spectrometer. Also, this is the type of analyzer implemented in our experiment. Such a detector provides major advantages if only low spectral resolution is required - photoelectrons with different kinetic energies can be analyzed simultaneously, thereby enhancing the sensitivity. 
The low duty cycle enhances the signal-to-noise ratio. Its equipment and properties will be represented in detail in subsection 4.6 .

\subsubsection{Sample handling}

Atoms and molecules can be investigated with UPS in the gas, liquid or solid phase. Methods for introducing the sample into the target chamber are therefore of great importance and vary considerably.

For gas-phase UPS there is a broad range of available techniques, for instance a slow bleed system for gases and volatile liquids, a fused capillary array giving a molecular beam or an atomic beam generated in a furnace. The sample handling for the investigation of liquids is more complicated. It is difficult to maintain a clean surface because of the large vapor pressure of liquids under vacuum conditions. Siegbahn and co-workers are pioneers who invented the first equipment to investigate liquids [14, 28]. They introduced the sample into the vacuum using a narrow liquid beam or a fine wire which is coated with the sample liquid. This is achieved by continuously moving the wire through a reservoir of the liquid. In several previous PES studies of liquids, samples were prepared using a rotating disc with a razor blade scraper to produce a clean and fresh surface in vacuum. Stainless steel needles and conical shaped trundles were also used in other experiments. In addition, highly concentrated salt solutions were used in order to lower the vapor pressure of the liquids. Another notable method is liquid micro-jet technique developed by Faubel et al. [29]. A thin liquid beam of $10-20 \mu \mathrm{m}$ diameter can be produced by pumping the liquid through a nozzle of the same diameter. We also apply this excellent method to probe liquids as well as gases, which will be shown extensively in subsection 4.4

\subsection{Theory of photoemission}

Photoelectrons show an intensity variation as a function of the angle of emission, because the polarization plane of the ionizing radiation defines an axis of quantization (see Fig.2.3). For unpolarized radiation the direction of the photon beam 
provides such an axis. It has been known for many decades that the angular dependence for electric-dipole induced transitions obeys the general law 30]

$$
I_{x}(\theta)=C\left[1+\beta P_{2}\left(\cos \theta_{x}\right)\right]
$$

where $x$ is the axis of polarization, $\theta_{x}$ is the angle between the momentum vector of the ejected electron and the $x$-axis, and $\beta$ is the asymmetry parameter or anisotropy parameter, which has been defined in various ways. $P_{2}\left(\cos \theta_{x}\right)$ is the second Legendre polynomial and is given by the expression $1 / 2\left(3 \cos ^{2} \theta_{x}-1\right)$. A corresponding expression for unpolarized radiation is given by Peshkin [31] as

$$
I(\theta)=C^{\prime}\left[1-\frac{1}{2} \beta P_{2}(\cos \theta)\right]
$$

where $\theta$ is now the angle between the trajectory of the ejected electron and the photon beam.

For atoms, the angular distribution of photoionization may be derived from quite general considerations of symmetry assuming that

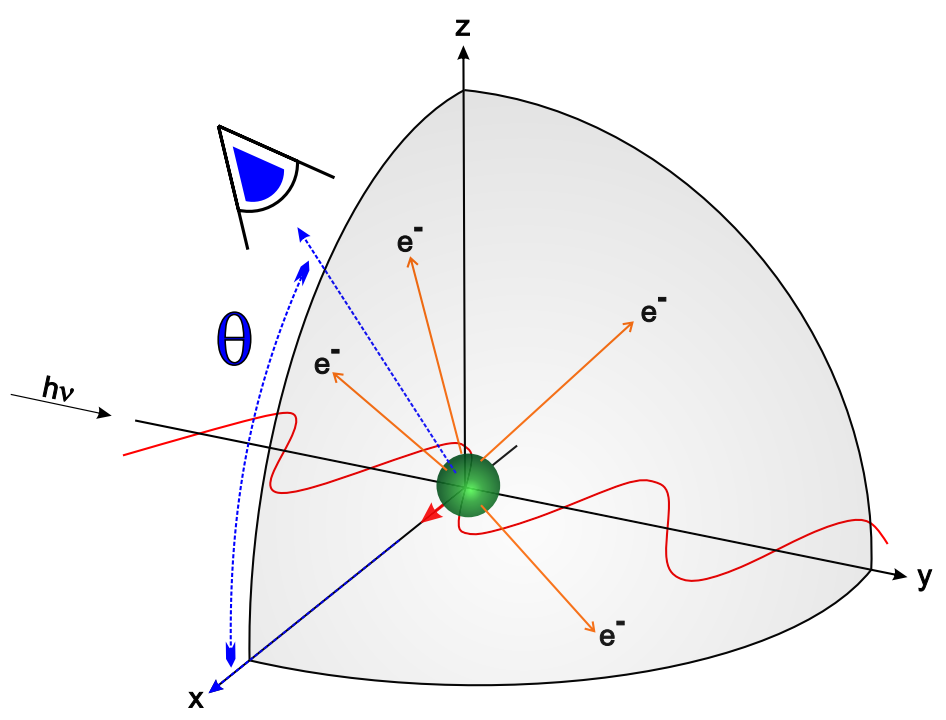

Figure 2.3: Angular distribution of photoelectron emission of a single atom. The polarization vector of the ionizing radiation is parallel to $x$-axis. $\theta$ is the angle between a particular trajectory of an ejected electron and the polarization axis of the incident radiation. 
* the target atoms are oriented at random,

* the influence of external fields is neglected and

* when more than one electron is emitted, the direction of emission of the second electron is independent of that of the first.

An expression for the asymmetry parameter $\beta$ in the photoionization process was first given by Bethe [30] for the hydrogen atom, where a central spherical potential field and LS coupling were assumed.

The measure of the probability of photoionization is the orbital-specific photoionization cross section which is included in the constant $C$ in equation 2.3. Within the dipole approximation, the differential photoionization cross section of a certain orbital $i$ is given by 32 ]

$$
\frac{d \sigma_{i}}{d \Omega}(\theta)=\frac{\sigma_{i}}{4 \pi}\left[1+\frac{\beta_{i}}{4}\left(1+3 P_{1} \cos 2 \theta\right)\right] .
$$

Here, $\sigma_{i}$ represents the total cross section which has a dimension of area and is a function of the incident photon energy. $\theta$ is the angle between the direction of the ejected electron and the polarization vector of the incident light. $P_{1}$ is the Stokes parameter and denotes the degree of linear polarization. For linearly polarized light $P_{1}=1$ and the above Eq. 2.5 can be transformed to

$$
\frac{d \sigma_{i}}{d \Omega}(\theta)=\frac{\sigma_{i}}{4 \pi}\left[1+\frac{\beta_{i}}{4}(1+3 \cos 2 \theta)\right]
$$

The value of the asymmetry parameter $\beta$, lies between 2 and -1 , and depends on the atomic system under consideration and the energy of the ejected electron. A simple approach to explain the angular distribution of photoelectrons from an atom is the selection rule for the angular momenta in electronic transitions: $\Delta l= \pm 1$ must be satisfied [33]. For instance, if an electron is removed from an s-orbital, it becomes an electron with $p$-character which has an angular distribution of a $\cos ^{2} \theta$-shaped function. But if a $p, d$, or higher order orbital is involved in the ionization, two partial waves represented by $l^{\prime}=l \pm 1$ must be considered. Under these conditions, interference terms may arise and $\beta$ will be smaller than 2 . This is equivalent with a broadening of the $\cos ^{2} \theta$-shaped distribution. Here, the value of $\beta$ depends on the velocity of the outgoing electron and increases as the velocity increases. 
In Fig.2.4, different angular distributions of photoionization are demonstrated for varying $\beta$ values $2,1,0$ and -1 but the same cross section $\sigma_{i}$ using linearly polarized light. At the magic angle $\theta=54.7^{\circ}$, the true magnitude of $\sigma_{i}$ can be obtained, since then, $d \sigma_{i} / d \Omega$ is not dependent on the $\beta$ value.

For molecules, the theory of the angular distribution is more complex than that for light atoms [34], since:

* The molecular orbitals are not eigenfunctions of the orbital angular momentum operator $l^{2}$, due to the absence of spherical symmetry.

* The bound orbital, out of which the photoelectron is ejected, is quantized on the molecular axis, while the continuum function of the ejected electron is more readily referred to space-fixed axes.

* There may be transfer of angular momentum between electronic and rotational motion.

However, experiments on the angular distribution of photoelectrons ejected from molecules by Berkowitz and Ehrhardt [35, 36] suggested that the angular distribution may be useful as a source of information regarding the symmetry of the molecular orbital from which photoelectrons of a given energy are ejected.

By determining the angular distribution for a molecular orbital, the angular momentum $l$ is not a good quantum number and consideration of symmetry must be made. For symmetrical molecules an evaluation of $l$ can be made in the following manner: When a molecule has an axial symmetry with reference to $z$-axis and a center of symmetry (taken to be the origin), then the molecular orbital should be represented by:

$$
\begin{gathered}
\psi=\sum_{l=m}^{\infty} R_{l}^{m}(r) P_{l}^{m}(\cos \theta)\left\{\begin{array}{c}
\cos m \phi \\
\sin m \phi
\end{array}\right\} \\
R_{l}^{m}(r)=\frac{2 l+1}{\left(2+2 \delta_{n_{0}}\right) \pi} \frac{(l-m) !}{(l+m) !} \int \psi P^{m}(\cos \theta)\left\{\begin{array}{c}
\cos m \phi \\
\sin m \phi
\end{array}\right\} \sin \theta d \theta d \phi
\end{gathered}
$$

where $m$ is a good quantum number and $n_{0}=2 m$ gives the number of nodes encountered when one goes around the azimuthal angle $\phi$. A molecular orbital with proper symmetry should have an even number of nodes. The molecular 


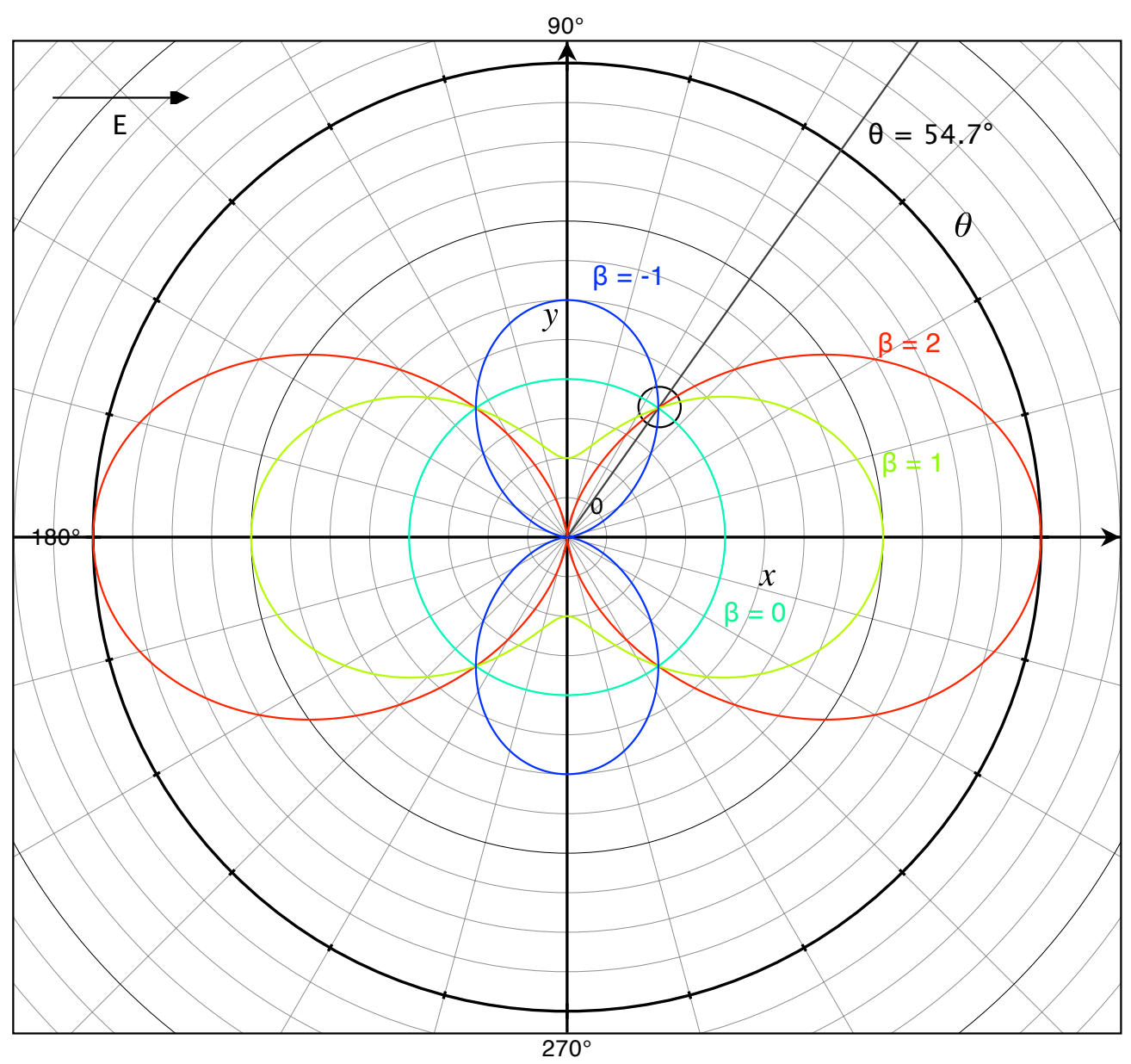

Figure 2.4: Angular distribution of photoionization associated with $\beta=2,1,0,-1$. The ionizing radiation is linearly polarized and propagates perpendicularly to the $x y$ plane through the origin. Polarization vector of the incident light and the magic angle $\theta=54.7^{\circ}$ are indicated, respectively. The $\sigma$ value is the same for all $\beta$ values. For $\beta=0$, the distribution of the photoelectron emission is isotropic. If $\beta>0$, most ejected electrons fly in the direction parallel to the polarization vector of the light, while the favored photoelectron detection angle is vertical to it, if $\beta$ is negative. 
orbital from the Hückel model should also follow this rule, at least approximately. $l$ can take any value $\geq m$, and is even for even parity and odd for odd parity. The most probable values of $l$ for a molecular orbital are the ones which have the largest value of $\left|R_{l}^{m}\right|$ at $r$, where $\left|R_{l}^{m}\right|$ reaches its maximum. For the valence orbitals of a simple molecule one expects only terms of lower $l$ to contribute significantly. By putting the molecular orbital $\psi$ into Eq.2.8, one may estimate the most probable values of $l$. Such calculations have been carried out for $\mathrm{N}_{2}, \mathrm{O}_{2}$, and benzene. Those calculations showed:

* The angular parameter $\beta$ for a given photoelectron band depends mostly on the nature of the initial orbital from which the photoelectron was ejected and not the different final states that may arise as the result of JahnTeller splitting, spin orbital splitting, or spin coupling between two unfilled orbitals.

* The relative intensities of the vibration bands for a given electronic band are generally independent of $\theta$, but in certain cases the vibrational spectrum appears to be strongly dependent on $\theta$.

To completely describe the photoionization process, $\beta, \sigma$ and photoelectron spin polarization are required [37]. If the ionizing radiation is linearly polarized, there is no dependence of $\sigma$ and the angular distribution on the spin state of the outgoing photoelectron, because any ejected electrons in all directions are unpolarized assuming the electric dipole approximation [38]. For circular polarized light the situation is different and much more complicated.

Many experiments focused on the determination of $\sigma$ or (and) $\beta$ parameters for atoms and small molecules within diverse photon energy ranges [37, 39, 40] while the investigation of photoelectron spin polarization was less common. By measuring the photon energy dependence of $\beta$ the so-called shape resonance can be determined [41].

The purpose of this work is to determine the $\beta$ and $\sigma$ values for varying systems (water and methanol) in different phases (gas, clusters and liquid). The interpretation of the obtained results will constitute the main evidence for understanding the electronic structures of these systems in altered phases. 


\section{Chapter 3}

\section{High Harmonic Generation}

\subsection{Introduction}

High harmonic generation (HHG) is a major branch of development in nonlinear optics and has attracted immense attention for over two decades [42, 43]. Ultrashort laser pulses in the extreme ultraviolet (EUV) and soft X-ray range can be obtained via HHG, which allow to resolve dynamics on the picosecond and femtosecond time scale. Since ten years, also the barrier towards attosecond pulses has fallen [44]. In the near future it may be possible to monitor electron dynamics with commercially available attosecond laser systems.

The essential goal of HHG is to efficiently generate high energy pulses from low ones. This can be achieved when a sequence of pulses, with low energy but high intensity, travel through a nonlinear media. By means of strong interactions with the media, a large number of photons can be converted to a few high-frequency photons which have multiples of the fundamental photon energy. These are the so called high harmonics. The nonlinear media can be atoms, small molecules, ions, plasma, solids or nano-structures.

In 1988 M. Ferray et al. performed the first experiments related to high harmonic generation [45]. However, their work did not immediately attract much attention. Using argon and xenon gases as the nonlinear media, they generated the 33rd harmonic $(32.2 \mathrm{~nm})$ of $1064 \mathrm{~nm}$ pulses from a Nd:YAG laser. Before that, experiments only demonstrated HHG up to the 11th harmonic [46. The breakthrough of high harmonic generation is closely related to the development of pulsed Ti:Sapphire laser systems [47]. Since their emergence, pulsed Ti:Sapphire 
laser systems gained expeditious development and popularity. The power has been increased from $10 \mathrm{~mW}$ to $10 \mathrm{~W}$ and the pulse duration can nowadays vary from a few $100 \mathrm{fs}$ to $8 \mathrm{fs}$. Currently, the photon energies achieved via high harmonic generation already exceed $200 \mathrm{eV}$ [48]. Also the HHG efficiency has been significantly improved. Recently, pulses with an intensity of $10^{6}$ photons/pulse at $100 \mathrm{eV}$ photon energy and a pulse duration of 170 as have been reported [49].

In section 3.2 it will be shown how we constructed and employed a HHG source with noble gases, to generate ionizing radiation for our UPS experiment. A new method for HHG using local field enhancement between nanometer-sized gold tips will be demonstrated in section 3.3 .

\subsection{HHG with noble gases}

High-harmonic generation by focusing a femtosecond laser onto a noble gas is a well-known method for producing coherent extreme-ultraviolet (EUV) light [45, 48, 50]. This nonlinear conversion process requires high pulse intensities, greater than $10^{13} \mathrm{~W} / \mathrm{cm}^{2}$. A well-known description of high harmonic generation within a semiclassical approach, the three-step model, was introduced by Corkum in 1993 [50]. This concept is applicable to describe the mechanism of the nonlinear interaction between a noble gas atom and a strong laser field, which will be illustrated in subsection 3.2.1.

\subsubsection{The three-step model}

One can visualize the three steps of HHG with the help of Fig. 3.1. In part a) the Coulomb potential $V(x)$ of a valence electron is deformed by the strong electrical field $E\left(x, t_{0}\right)$ of the electromagnetic light wave which is linearly polarized. The superposition of both potentials results in a potential surface $V(x)-e x E\left(x, t_{0}\right)$ which is bend down. With a low probability, the electron can tunnel out of the bent potential, when the field force of the light is temporarily larger than the Coulomb force. Within the three-step model, an electron which left its parent atom via tunnel ionization is considered as a free electron. This means that the atomic Coulomb field does not influence it any more. The motion of the ionized 
electron in the laser field is then describable with classical mechanics. Right after leaving the core, the electron starts $\left(t_{0}\right)$ traveling in the laser force field with a starting velocity of $v_{0}=0$.

At the initial time $t_{0}$, the phase of the electric field is $\phi_{0}=\omega t_{0}$. There are two different courses of the free electrons which must be distinguished. In the first case, $t_{0}$ lies between a zero-crossing and a subsequent maximum of the electric field $n \pi<\phi_{0}<\left(n+\frac{1}{2}\right) \pi(n=0,1,2, \ldots)$. In this case, the electron is accelerated consistently away from its parent atom and never returns. In the second case, the electron starts at phase $\left(n-\frac{1}{2}\right) \pi<\phi_{0}<n \pi$ and is accelerated away from its parent core by the laser field force until the latter changes its sign at the zerocrossing $\phi=n \pi$. Past this point, the electron is slowed down until it has zero kinetic energy at the time $t_{u}$ or the phase $\phi_{u}=2 n \pi-\phi_{0}$. From now on, the electric field acts on the electron as an accelerator and drives it back to its original core. At the time $t_{1}$ the electron recombines with the core by emitting a photon
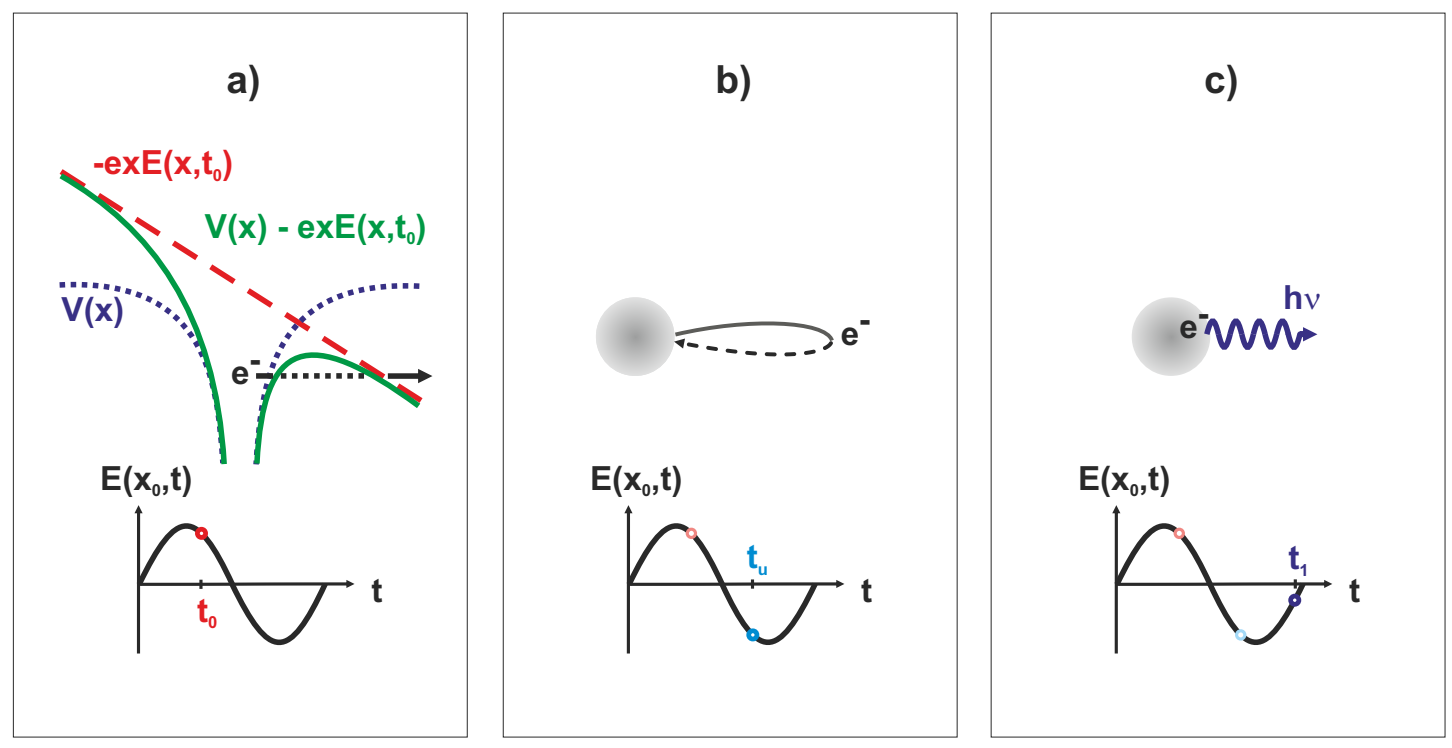

Figure 3.1: Semiclassical three-step model for high harmonic generation. a) An electron leaves the core via tunnel ionization at $t_{0}$. b) It is accelerated by the electric field of the laser light and turns back towards its parent atom at $t_{u}$ and c) recombines with the latter at $t_{1}$ by releasing a high harmonic photon. 
(see c) in Fig. 3.1. This photon obtains the released excess kinetic energy of the returned electron.

The recombination event competes against other possible scenarios. One of them is elastic scattering connected to the so-called above threshold ionization (ATI) [51]. The other is inelastic scattering with the remaining electrons of the parent atom. In this case, a second electron is ejected from the system resulting in a doubly ionized species. This process is known as non-sequential double ionization (NSDI) [52.

In the recombination of the electron with its parent atom, the energy of the released photon can be determined with classical mechanics. It is the sum of the electron kinetic energy $E_{k i n}\left(t_{1}\right)$ at the time of recombination and the ionization potential $I_{p}$ of the atom. At the time $t_{1}$, the electron possesses a kinetic energy between 0 and $3.17 U_{p}$, which depends on $t_{0}$. The ponderomotive potential $U_{p}$ is the average kinetic energy of the electron in the laser field and can be estimated as

$$
U_{p}=\frac{e^{2} E_{L}^{2}}{m_{e} \omega_{0}^{2}}=\text { const } I_{L} \lambda_{0}^{2} .
$$

where $e$ is the elementary charge and $m_{e}$ is the rest mass of an electron. $E_{L}$ and $I_{L}$ are the amplitude and intensity of the electric laser field. $\lambda_{0}$ is the central wavelength and $\omega_{0}$ the respective angular frequency. The maximum energy of the high harmonic photons and thus the experimentally observed cutoff energy in the high harmonic spectrum (see Fig. 3.2p yields

$$
E_{\max }^{\text {Photon }}=\hbar \omega_{\max }=3.17 U_{p}+I_{p} .
$$

This maximum photon energy can only be attained if the tunnel ionization of the electron takes place at the particular phase $\phi_{0}=0.67 \pi$ of the electric field. In this case, the reversal point $t_{u}$ is at a phase $\phi_{u}=1.4 \pi$ and the recombination $\left(t_{1}\right)$ at $\phi_{1}=1.9 \pi$. The bottom part of Fig. 3.1 illustrates this case.

The quantum mechanical description accompanying the semiclassical model bases upon solving the non-relativistic time-dependent Schrödinger equation for an electron in the electrical field $E(x, t)$ of the laser light [53]. In the position-space, the equation is:

$$
i \hbar \frac{\partial \Psi(x, t)}{\partial t}=\left(-\frac{\hbar^{2}}{2 m} \frac{\partial^{2}}{\partial^{2} x}+\hat{V}(x)-e x E(x, t)\right) \Psi(x, t)
$$


where $\Psi(x, t)$ designates the electrons wave function in the position-space, $\hat{V}(x)$ represents the time-independent Coulomb potential of the atom and $-e x E(x, t)$ the interaction of the electron with the laser field. The goal is to calculate the timedependent dipole moment $d(t)$ induced by the interaction of the electron with the electric field. Therefor, one has to integrate over all possible times $t_{0}$ when an electron becomes free via tunnel ionization, and take all the resulting trajectories into account. The spectrum of the high harmonics as shown in Fig. 3.2 is obtained by squaring the absolute value of the Fourier transformation $d(\omega)$ of the time dependent dipole moment $d(t)$ [43].

In oder to solve Eq. 3.3 , a strong field approximation must be provided which is valid for $U_{p}>I_{p} \gg \hbar \omega_{0}$. Upon this approximation, the electron is considered as a non-bound particle after the tunnel ionization. It means that the Coulomb field of the core is neglected once the electron left the atom. The second assumption is

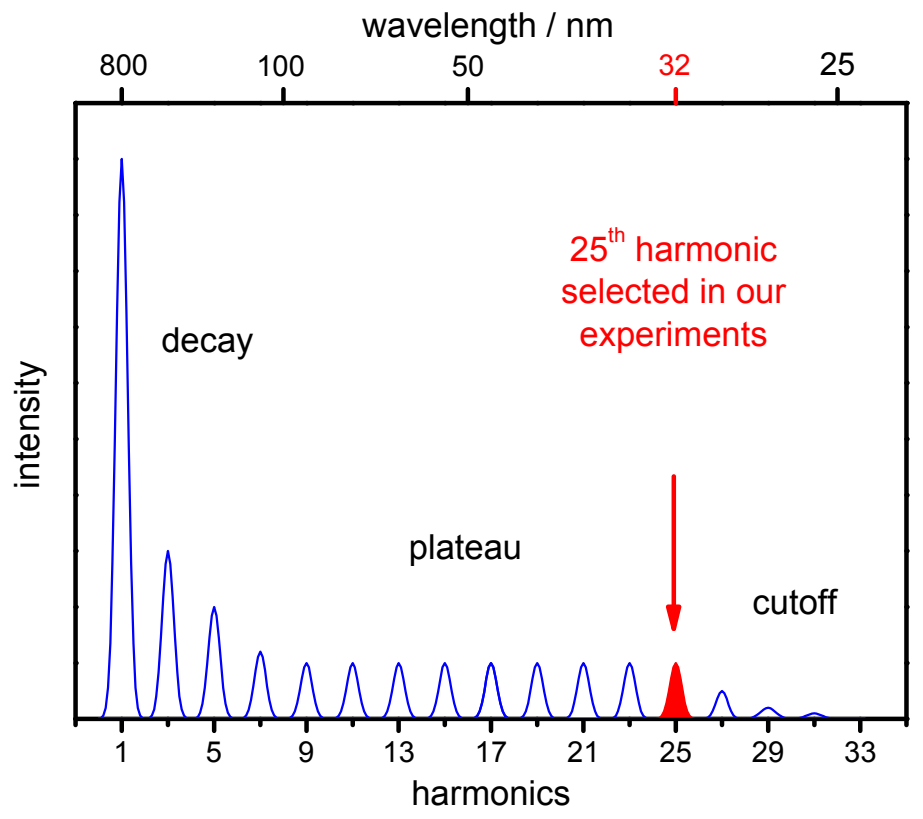

Figure 3.2: Schematic spectrum of the high harmonics generated in our experiment. In this experiment the selected ionizing radiation is the $25^{\text {th }}$ harmonic of the $800 \mathrm{~nm}$ fundamental light, which corresponds to a photon energy of $38.7 \mathrm{eV}$ or a wavelength of $32.0 \mathrm{~nm}$. 
that the electron only recombines with the original atom and further goes back to its initial state. Other possible final states are not considered.

The semiclassical three-step model can only explicate the interaction of a single atom in a laser field. Collective effects in high harmonic generation, such as phase matching conditions, are not considered, although these effects are important for the understanding of the strength of macroscopic high harmonic emission. Additionally, this model is not applicable for the description of the quantum nature of the electrons' interaction with the laser field, and thus that of high harmonic generation in general. Despite those weaknesses, the three-step model is broadly accepted because of its simplicity to describe the main properties of high harmonic generation such as the spectral features and the polarization characteristics.

\subsubsection{The EUV source}

In this experiment we generate high harmonic radiation by focusing $1.25 \mathrm{~W}$ of the $800 \mathrm{~nm}$ fundamental pulses (see section 4.1) through a closed-end metal capillary perfused by argon gas (see Fig. 3.3. . Therefor, a lens with a focus of $\mathrm{f}=30 \mathrm{~cm}$ is used which focuses the fundamental light $(\varnothing=7-8 \mathrm{~cm})$ down to a diameter of about $50 \mu \mathrm{m}$. Since the size of the beam diameter was found to be critical for the quality and the intensity of the high harmonic radiation, we use an adjustable iris right in front of the focusing lens for optimization. Within the focus, a high intensity of $8 \cdot 10^{14} \mathrm{~W} / \mathrm{cm}^{2}$ is achieved. The capillary consists of an alloy of copper, nickel and zinc, and has an inner diameter of $5 \mathrm{~mm}$. The holes $(\varnothing=200 \mu \mathrm{m})$ for the Ar-gas effusion out from the capillary arise from melting off the material by contact with the highly intensive $800 \mathrm{~nm}$ radiation. The capillary system arranged in a vacuum chamber (called HHG chamber) is connected with an Ar-gas cylinder. For the optimal generation condition of high harmonics, the pre-pressure of this Ar-gas cylinder is $100-200$ mbar. During the experiment, a roots and a rotary vane pump maintain the pressure in the HHG chamber at about $10^{-2}$ mbar.

To separate harmonics of different order and further to select one particular harmonic, we use a toroidal holographic EUV grating ( $\mathrm{Au}, 600$ lines $/ \mathrm{mm}, 12-36 \mathrm{~nm}$, Jobin Yvon), positioned in the neighboring chamber. A $150 \mathrm{~nm}$ thick aluminum filter (Luxel) between the two chambers blocks the fundamental radiation and serves 


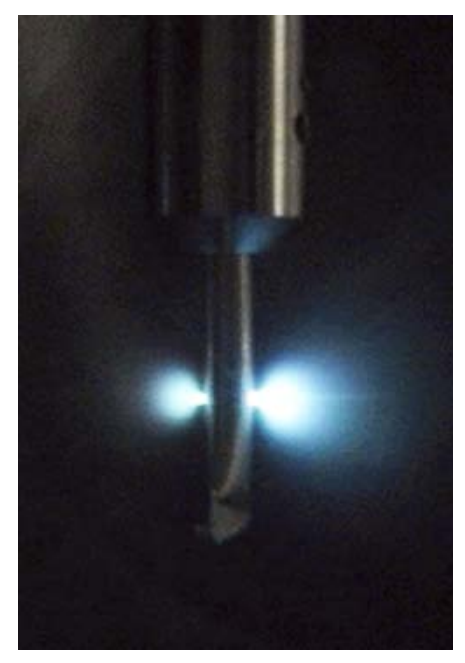

Figure 3.3: High harmonic generation by focussing the $800 \mathrm{~nm}$ fundamental light pulses into argon gas escaping from a metal capillary, whereby plasma fluorescence can be observed, resulting from the hole burning process.

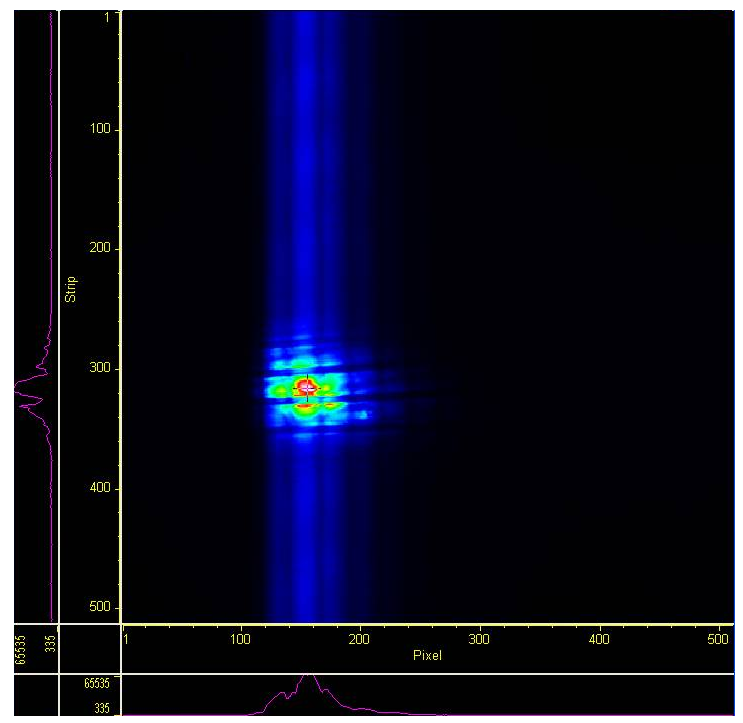

Figure 3.4: CCD camera image of the $25^{\text {th }}$ harmonic. The vertical and horizontal lines are associated with the projection of an aluminum filter, which is installed between the CCD-camera and the main chamber. Evaluation of the image allows to estimate the intensity and the focus size of the high harmonic radiation. 
as a pressure shield, additionally. The grooves of the grating are computer optimized to provide a point-to-point image with a minimum of astigmatism and coma. The grating focuses the selected harmonic down to a spot size of about $200 \mu \mathrm{m}$ at a distance of $1414 \mathrm{~mm}$. The distance between the high harmonic capillary and the grating is $1000 \mathrm{~mm}$.

\subsubsection{Characteristics of high harmonics}

Conversion efficiency of HHG For monitoring and optimizing the high harmonic radiation we use a CCD camera (Princeton instruments). The camera is attached to the main experimental chamber (see Fig.4.3). A typical image is shown in Fig. 3.4. Evaluation of the data allows to estimate the number of high harmonic photons generated in our high harmonic light source. In the $25^{\text {th }}$ harmonic we generate a total number of around $6 \cdot 10^{6}$ photons per pulse.

Harmonic emission pulse length In 1996 Schins et al. confirmed the prediction of HHG temporal profiles in the early calculations and proved this suggesting "... harmonics are created only during the rising edge of the fundamental pulse" [54]. A great deal of experimental results are consistent with the theoretical expectation [55, 56]. Particularly, Glover and co-workers investigated carefully a similar generation method as used in our EUV source and yielded a pulse duration of $50 \pm 15 \mathrm{fs}$ for the $23 \mathrm{rd}$ harmonic of $800 \mathrm{~nm}$ for $70 \mathrm{fs}$ driver pulses. Referring to this work, we estimate that the high harmonics generated with our radiation source should be about $80 \mathrm{fs}$ short since the fundamental $800 \mathrm{~nm}$ pulses have a duration of $110 \mathrm{fs}$. Unfortunately, the drawback of such a simple grating unit as the one used in our apparatus, is the temporal broadening of the high harmonic pulses. After the grating, the pulse length is temporally broadened to about 1 ps but the spectral profile is narrow, as designated.

Polarization properties Another essential property of HHG is the polarization of the harmonics. The ellipticity of the laser pulses is very crucial for the efficiency of the HHG due to the nature of the harmonic generation mechanism itself [57, 58]. Early experiments [59] with a fundamental wavelength of $10.6 \mathrm{~mm}\left(\mathrm{CO}_{2}\right.$ laser) showed that the visible harmonics are linearly polarized, with $90 \%$ of the 
harmonic radiation polarized parallel to the driving (fundamental) optical field. Measurements reported by Budil and co-workers [60] in 1993 exhibited a rapid decline in harmonic generation efficiency as the fundamental field ellipticity was increased. Notice that ellipticities of only $0.1-0.2$ are sufficient to reduce the photon yield by a factor of two. Because of the high conversion efficiency in our HHG source, we believe that the generated high harmonics are over $99 \%$ linearly polarized - in the same way as the fundamental light.

\subsection{HHG with nano-structures}

Developments of HHG with nano-structures are still at the very beginning. Nevertheless, we might forecast that nano-structures will be the favorite subjects in the future, since compared to the other nonlinear media, they have unparalleled advantages:

* Nano-structures can be engineered systematically.

* They have a much larger cross section and higher density of states for efficient harmonic generation and

* a much weaker laser intensity is required.

However, there are also a lot of noticeable disadvantages of nano structures, which make their development to a real challenge: easy fragmentation, strong internal absorption of $\mathrm{HH}$ and enhanced multiple electron emissions and ionizations [61.

Up to now, a few short polyenes have been investigated experimentally, although the highest harmonic order is still below the 21st. Nanotubes, polyenes and fullerene C60 have been theoretically examined, but substantial experimental efforts are still missing [61].

In $2008 \mathrm{Kim}$ et al. demonstrated an experimental approach to modify a conventional HHG approach with Ar as medium. In contrast to the common HHG sources with noble gases, they used additionally a nanostructure consisting of gold bow-tie elements on a thin sapphire substrate [62]. The nonlinear conversion process for HHG in a noble gas requires high pulse intensities, greater than $10^{13} \mathrm{~W} / \mathrm{cm}^{2}$, which are not directly available using only the output of a femtosecond oscillator. But 
as a result of the local field enhancement induced by the nano-structure, the high laser intensity of $10^{13} \mathrm{~W} / \mathrm{cm}^{2}$ was not necessary any more - only a Ti:Sa oscillator was used, which generated $10 \mathrm{fs}$ short pulses with intensities of $10^{11} \mathrm{~W} / \mathrm{cm}^{2}$. They supposed that this method could form the basis for constructing laptop-sized EUV light sources for advanced lithography and high-resolution imaging applications. We are also interested in such subject and concern ourselves with the development of a novel method to invent an EUV source which requires only low incoming light intensity, and is therefore more compact than the existing ones.

\subsubsection{Construction of nano-structures}

Resonant plasmons can be induced on a metallic nanostructure, when ultrashort laser pulses with a duration of less than or near $10 \mathrm{fs}$ pass through the nanostructure and interact with it. In narrowly localized regions within the nanostructure, collective motion of free electrons occurs, which results in field enhancement. The similar effects were also revealed in previous investigations of different shapes of particles, wires and tips [63 66]. For the given geometry of a nano-particle or nano-structure, the degree of enhancement induced by resonant plasmons can be estimated by solving Maxwell's equations analytically or numerically. In reverse order, one can also design a nanostructure by considering the degree of field enhancement.

Using focused ion beam (FIB) technique, we fabricate a nano-structure on gold film (50 nm thick) adsorbed on a $500 \mu \mathrm{m}$ mica plate. Numerous bow-tie elements

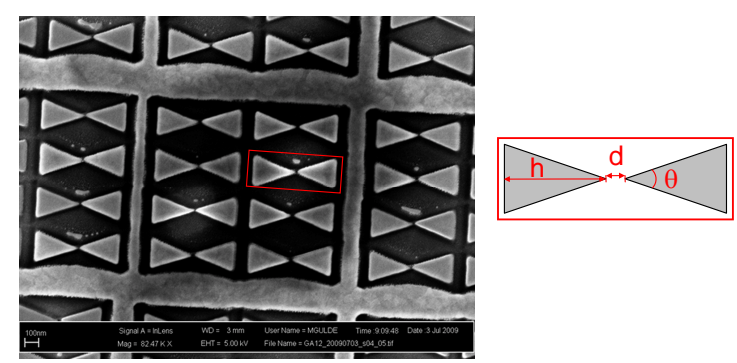

Figure 3.5: The SEM image of a nano-structure used in this work. The characteristic parameters are: $h=300 \mathrm{~nm}, t=50 \mathrm{~nm}, \theta=40^{\circ}$ and $d=30 \mathrm{~nm}$. 
in a two-dimensional array are ordered over a $10 \mathrm{~mm} \times 10 \mathrm{~mm}$ area in the middle of the gold film. The SEM (scanning electron microscope) image of one of the applied nano-structures is shown in Fig. 3.5. Similar to Kim et al., we take the bow-tie shaped nanostructure element as basic feature. It is a pair of triangular patches placed apex to apex with a small gap between them. Exposed to the femtosecond pulses, free electrons are confined at one apex of the bow-tie element while the opposing apex is filled with positive charges. Then the electric field of the ultrashort pulse can be enhanced between the vertices. As illustrated in Fig. 3.5. the bow-tie shaped basic feather is characterized by the following geometrical parameters: the height $(h)$, the angle $(\theta)$ and the gap $(d)$ between the vertices. Also the thickness $(t)$ of the gold film is crucial for the field enhancement. The characterizing geometrical parameters of the nano-structure shown here are $h=300 \mathrm{~nm}, t=50 \mathrm{~nm}, \theta=40^{\circ}$ and $d=30 \mathrm{~nm}$, which is comparable with the parameters in 62 .

\subsubsection{8fs-Oscillator system}

As source of the fundamental light for the HHG, a $<8 \mathrm{fs}$ oscillator (VENTEON | PULSE : ONE, VENTEON Laser Technologies) is employed, which has a modelocked output of $400 \mathrm{~mW}(80 \mathrm{MHz}$ rep rate) at a central wavelength of $800 \mathrm{~nm}$ (FWHM $>200 \mathrm{~nm})$. The oscillator is pumped by a frequency doubled Nd:YAG laser (Verdi V-5, Coherent, $532 \mathrm{~nm}, 5 \mathrm{~W}, 1 \mathrm{kHz}$ ). The Nd:YAG light beam is turned by a periscope to have a polarization parallel to the optical table plane and a height of $100 \mathrm{~mm}$ above that.

A schematic view of the optical arrangement of the oscillator is shown in Fig. 3.6. Two mirrors are used to adjust the incoming direction of the pump beam. The main unit of the oscillator is a cavity with a Ti:Sapphire crystal in the center. The Ti:Sa crystal is cooled by a chiller to a constant temperature of $17^{\circ} \mathrm{C}$. The mode-locked operation has to be started with a mechanical switch. To optimize the output and spectral width, the cavity length can be changed by tuning the translation stage of M3 and also the wedge (W) position by fine tuning the dispersion. After each change in M3 position, wedge position or pump power, the output coupler (OC) 
needs to be adjusted to maximize $\mathrm{CW}$ power. The shortest pulse duration $<8$ fs will be achieved at a distance of $0.5 \mathrm{~m}$ from the exit.

\subsubsection{Experimental setup}

As shown in Fig. 3.7, the mica plate is fixed in the HHG chamber. The position of the sample plate can be varied with a three-dimensionally motorized holder, which is not displayed in this figure. The nano-structure is on the back side of the mica plate facing a quartz nozzle $(\varnothing=100 \mu \mathrm{m})$ of the gas jet. The nozzle also can be moved in three spatial directions to adjust the optimal distance between nanostructure and nozzle. Before the output beam of the $8 \mathrm{fs}$-oscillator is guided into the HHG chamber, a chirped mirror pair and wedges compensate the group delay

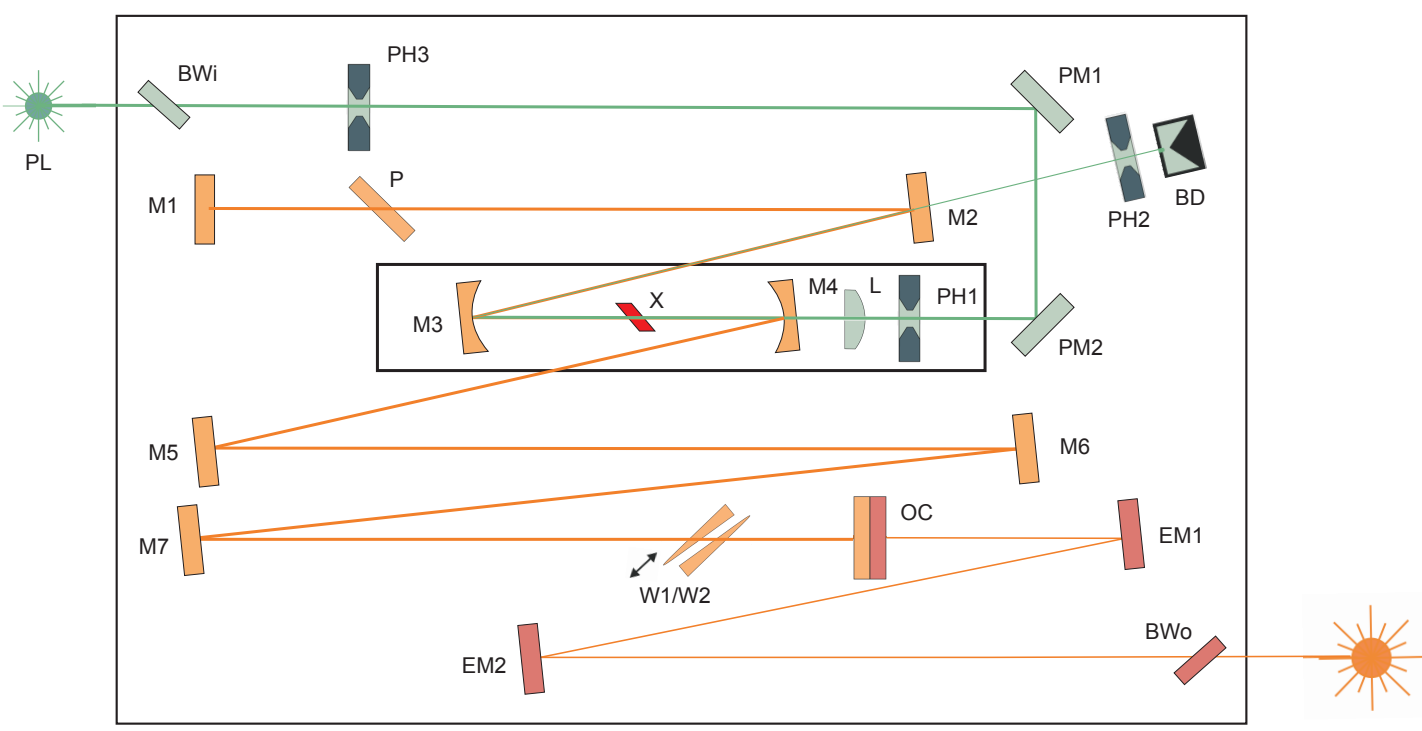

Figure 3.6: Optical Setup of VENTEON | PULSE : ONE. (PL) = Pump Laser, (BWi) $=$ Input Brewster Window, $(\mathrm{PM} 1),(\mathrm{PM} 2)=$ Pump Alignment Mirrors, $(\mathrm{L})=$ Focussing Optics, $(\mathrm{BD})=$ Pump Beam Dump, $(\mathrm{PH} 1)-(\mathrm{PH} 3)=$ Adjustment Pinholes, $(\mathrm{M} 1)-$ $(\mathrm{M} 7)=$ Cavity Mirrors, $(\mathrm{X})=$ Ti:Sa Crystal, $(\mathrm{P})=\mathrm{BaF}_{2}$ Plate, $(\mathrm{W})=$ Dispersion Tuning Wedges, (OC) = Output Coupler, (EM1), (EM2) = Extracavity Mirrors, (BWo) $=$ Output Brewster Window. 
dispersion of the pulses, which is caused by the progression in air. Then the $800 \mathrm{~nm}$ pulses are expanded by a two-spherical-mirror telescope to be sharply focused by a spherical mirror subsequently. The focused beam enters the vacuum chamber and passes through the nano-structure on the mica plate. In the interaction area with the nano-structure, the beam focus is around $16 \mu \mathrm{m}$, according to an initial laser pulse intensity of some $10^{11} \mathrm{~W} / \mathrm{cm}^{2}$. Assuming a field enhancement by a factor of 100 via the nano-structure, the final pulse intensity is increased to $10^{13} \mathrm{~W} / \mathrm{cm}^{2}$.

The same motorized toroidal holographic EUV grating, as mentioned above, is used to diffract the generated HH's for the detection via a Multi Channel Plate (MCP) combined with a Charge-Coupled Device (CCD) camera. The MCP photon multiplier is located at a distance of $700 \mathrm{~mm}$ from the grating.

\subsubsection{Preliminary results and outlook}

First 0th order measurements - in which the generated harmonics with different orders are not separated - are shown in Fig. 3.8, 3.9 and 3.10, whereby all the generated harmonic orders are not separated. We observed photons with high energy, which clearly results from the field enhancement by the nano-structure. Fig. 3.8 is one picture (100 ms integration time) from the movie sequence recorded during a measurement. In addition, a dependence of the high harmonic signal on the Ar-gas pre-pressure was revealed, as demonstrated in Fig.3.9.

Unfortunately, we could not separate harmonics of different orders because of the geometry of the grating construction. We merely know that the orders of the harmonics must be under 11th, since the photon intensity disappeared when the Al-filter between the HHG and grating chamber was inset, which allows only photons with energies over $18 \mathrm{eV}$ to penetrate. This is shown in Fig. 3.10. With a new setup, it will be possible to separate single harmonic orders and to determine specific efficiency of generation for each of them. This is our goal for the near future.

For further development of this method, the following important subjects must be regarded intensively: 


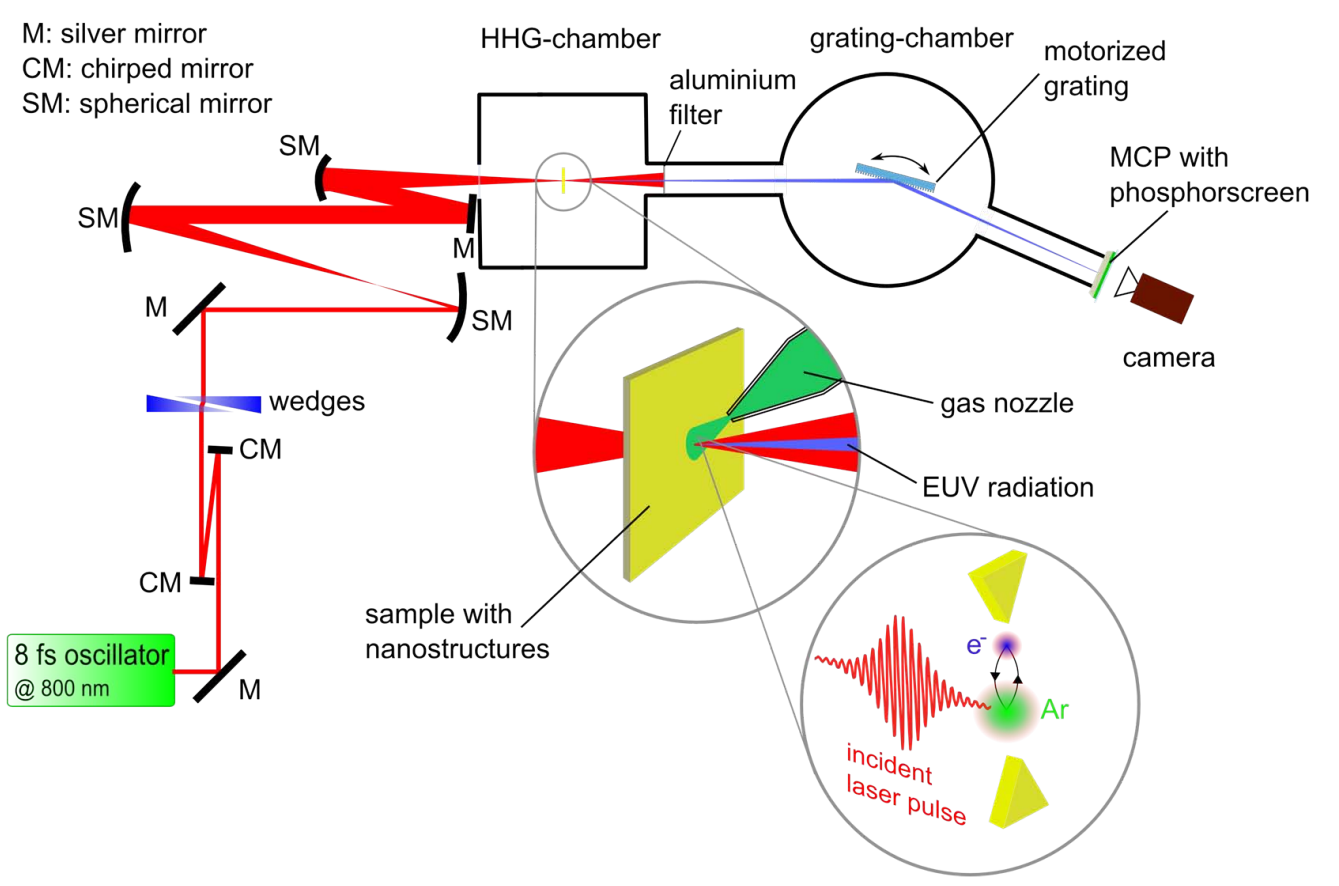

Figure 3.7: Experimental setup of HHG exploiting nano-structure. The used quartz nozzle has a diameter of $100 \mu \mathrm{m}$. The beam focus is around $16 \mu \mathrm{m}$ in the interaction range with the nano-structure. The pulse intensity focused on the nano-structure amounts for $10^{11} \mathrm{~W} / \mathrm{cm}^{2}$ without the field enhancement, and $10^{13} \mathrm{~W} / \mathrm{cm}^{2}$ with it.

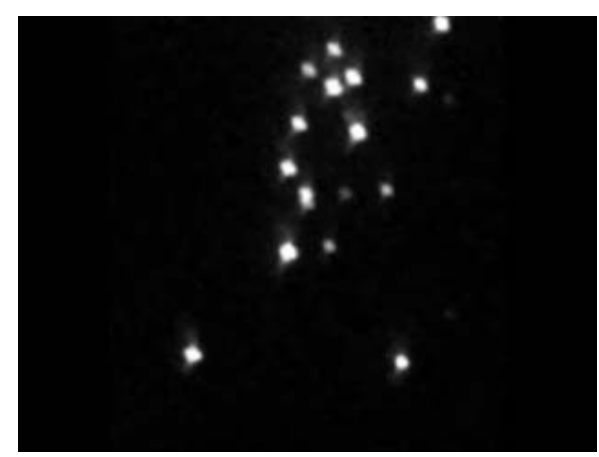

Figure 3.8: One picture ( $100 \mathrm{~ms}$ integration time) from the movie sequence recorded during a measurement. Sample: GA19-4. 


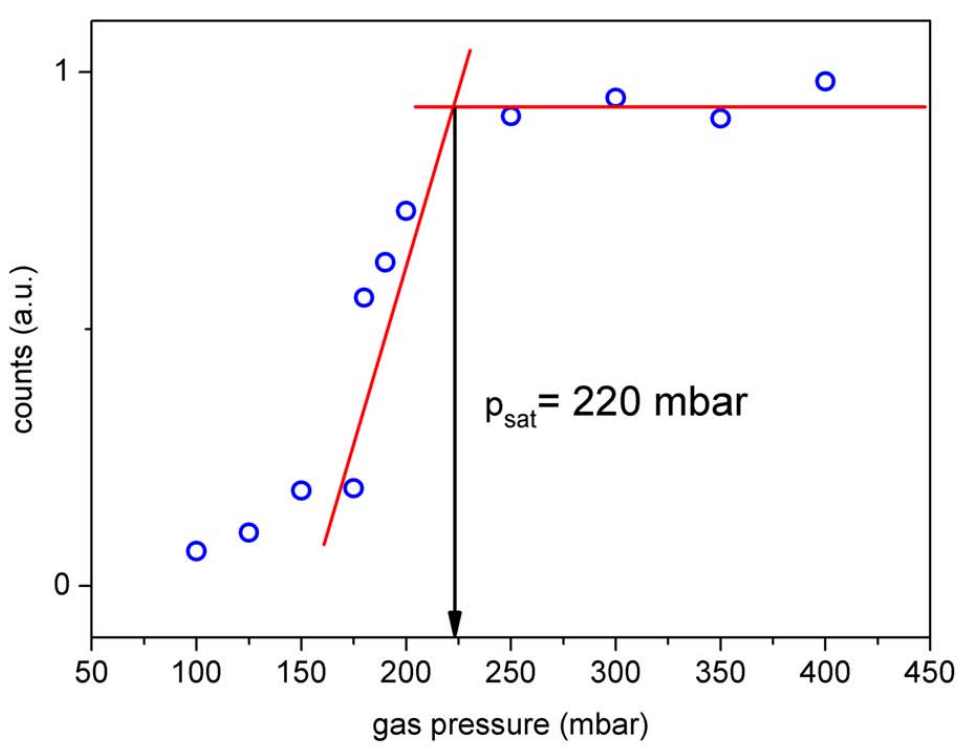

Figure 3.9: HHG efficiency dependence on the Ar pre-pressure. No significant harmonics photons are observable at low Ar densities ( $<175$ mbar). Above 220 mbar the harmonics intensity reaches its saturation and does not increase any further.

* Concerning the grating properties and the detection instruments, our present setup is not very suitable for such an experiment. A new setup is currently designed which allows the separation of the various harmonics.

* The fabrication procedure can probably be optimized to obtain nano-structures for higher field enhancement factors. The distance between the two vertices is particularly critical, which presents a challenge for the creation of nano-structures using FIB technique.

* Another essential challenge is to devise novel sample attaching techniques in order to reduce thermal damage to the sample. In Fig. 3.11 the temporal trend of the damage process is illustrated. After $80 \mathrm{~min}$ the sample was seriously damaged. Other types of substrate or coating may be the solution of this problem. 


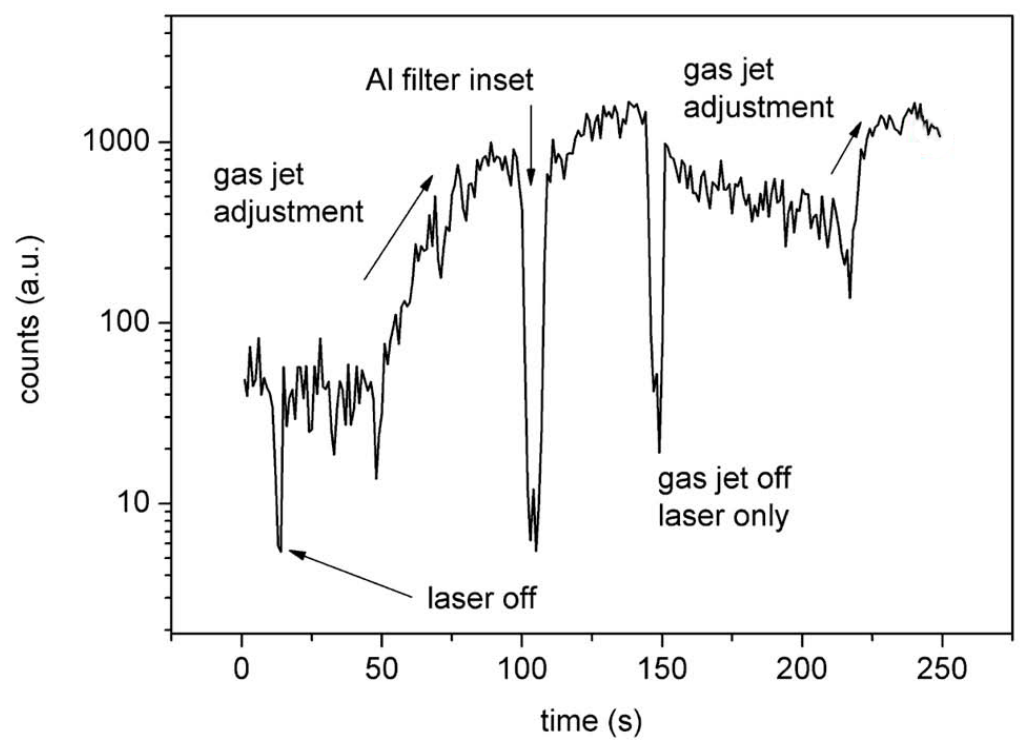

Figure 3.10: Evidence for the successful HHG in the presence of our nano-structure. Only when both the laser and Ar-gas flux are on, significantly multiplied high energy photons can be detected. If the Al-filter is inserted into the propagation path of the radiation, no high energy photons reach the MCP, which indicates - the orders of the generated harmonics are $<11$ th. 


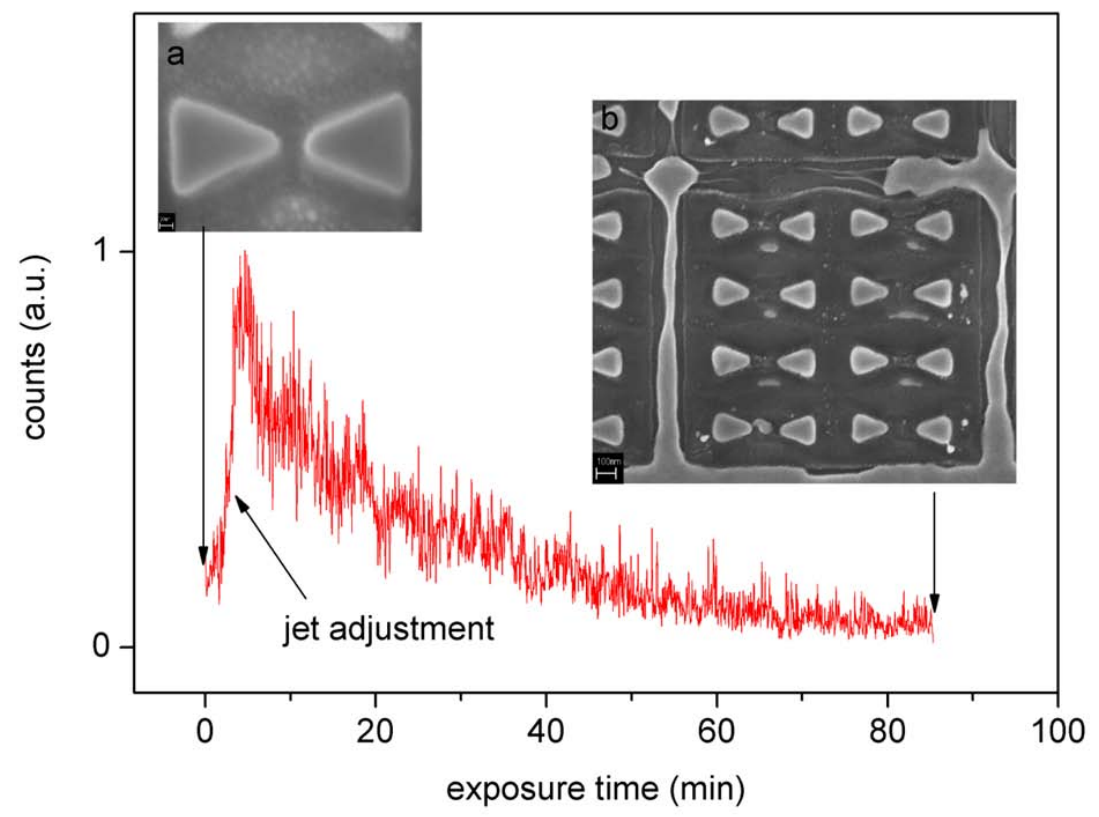

Figure 3.11: Temporal trend of the damage process on the nano-structure. a) is the SEM close-up image of an unused nano-structure sample. b) The sample withstood 80 min laser exposure. 



\section{Chapter 4}

\section{Experimental approach}

Up to the present, there have not been any experimental determinations of the anisotropy parameters for the molecular valence orbitals in liquid water or methanol. This lack is due to the challenging requirements for the construction of an applicable experimental setup, which will be shown in the following.

For the investigation of the angular distribution of photoelectron emission, measurements at different angles $\theta$ between the photoelectron detecting direction and the polarization vector of the ionizing radiation (see section 2.4 have to be made. The adjustment of angle $\theta$ can be realized by rotating either the axis of the spectrometer or the polarization vector of the incident light. If the spectrometer in the experimental setup is fixed, a suitable radiation source with changeable polarization is needed. At a synchrotron radiation beamline, only few end stations can serve this purpose. In our experiment, we generate the EUV ionizing radiation via HHG and the polarization of the EUV radiation can be easily tuned (see section 4.2.

In addition, the PES investigation of a sample in the liquid phase itself is challenging, because of the difficulty to obtain a clean liquid surface in vacuum. Using the liquid micro-jet technique, we are able to fulfill this criterium and to carry out angle-dependent PES studies upon stable experimental conditions (see section 4.4). 


\subsection{The laser system}

There are several paths until the ionizing photon beam is generated for the PES experiment finally. Our complete laser beam line is presented in Fig.4.1, which will be separately described in the present section.

Hurricane $^{\mathrm{TM}}$ Ti:Sa system To generate EUV radiation as ionizing source, we apply the high harmonic generation technique with Ar as medium which has been described in detail in section 3.2. We use a commercial titanium sapphire (Ti:Sa) laser system Hurricane ${ }^{\mathrm{TM}}$ (Spectra Physics) as source of $800 \mathrm{~nm}$ femtosecond fundamental pulses. The system delivers $100 \mathrm{fs}$ pulses with a pulse energy of $1.0 \mathrm{~mJ}$ and a repetition rate of $1 \mathrm{kHz}$ at a central wavelength of $800 \mathrm{~nm}$. However, in the present experiments we use $1 / 4$ of the uncompressed output $(0.2 \mathrm{~mJ}, 800 \mathrm{~nm}$, $1 \mathrm{kHz}$ ), guide it through an additional multi-pass amplification unit and compress the amplified pulse with a two grating geometry to generate $100 \mathrm{fs}$ pulses of $1.2 \mathrm{~mJ}$ energy ( $800 \mathrm{~nm}$ central wavelength). The rest of the Hurricane ${ }^{\mathrm{TM}}$ laser energy is required to generate pump pulses for other time-resolved UPS experiments [9, 67].

The Ti:Sa laser system Hurricane ${ }^{\mathrm{TM}}$ is based on the chirped pulse amplification (CPA) method and consists of four main components: oscillator, pulse stretcher, regenerative amplifier and pulse compressor. Detailed descriptions can be found in references [68, 69].

The oscillator (Mai Tai ${ }^{\mathrm{TM}}$ ) consists of two components: a resonator with a Ti:Sa crystal in the middle of it and a frequency doubled cw Nd:YAG laser (Millennia ${ }^{\mathrm{TM}}$ ). The spectral range of the Millennia ${ }^{\mathrm{TM}}$ is tunable from 690 to $1064 \mathrm{~nm}$ which makes it possible to vary the central Mai Tai ${ }^{\mathrm{TM}}$ wavelength from 780 to $820 \mathrm{~nm}$. A short disturbance of the resonator geometry induces the mode locking of multiple resonator modes which results in ultrashort laser pulses of $90 \mathrm{fs}$ and $1 \mathrm{~W}$ output. The pulses leave the resonator and pass a pulse stretching unit consisting of a grating and multiple mirrors. The resulting pulse length is in the order of a few picoseconds, which is necessary in order to avoid damage of optical elements in the regenerative amplifier.

The regenerative amplifier unit, which was described in [68] in detail, is a cavity resonator with another Ti:sapphire crystal in the center of it. A frequency doubled, 


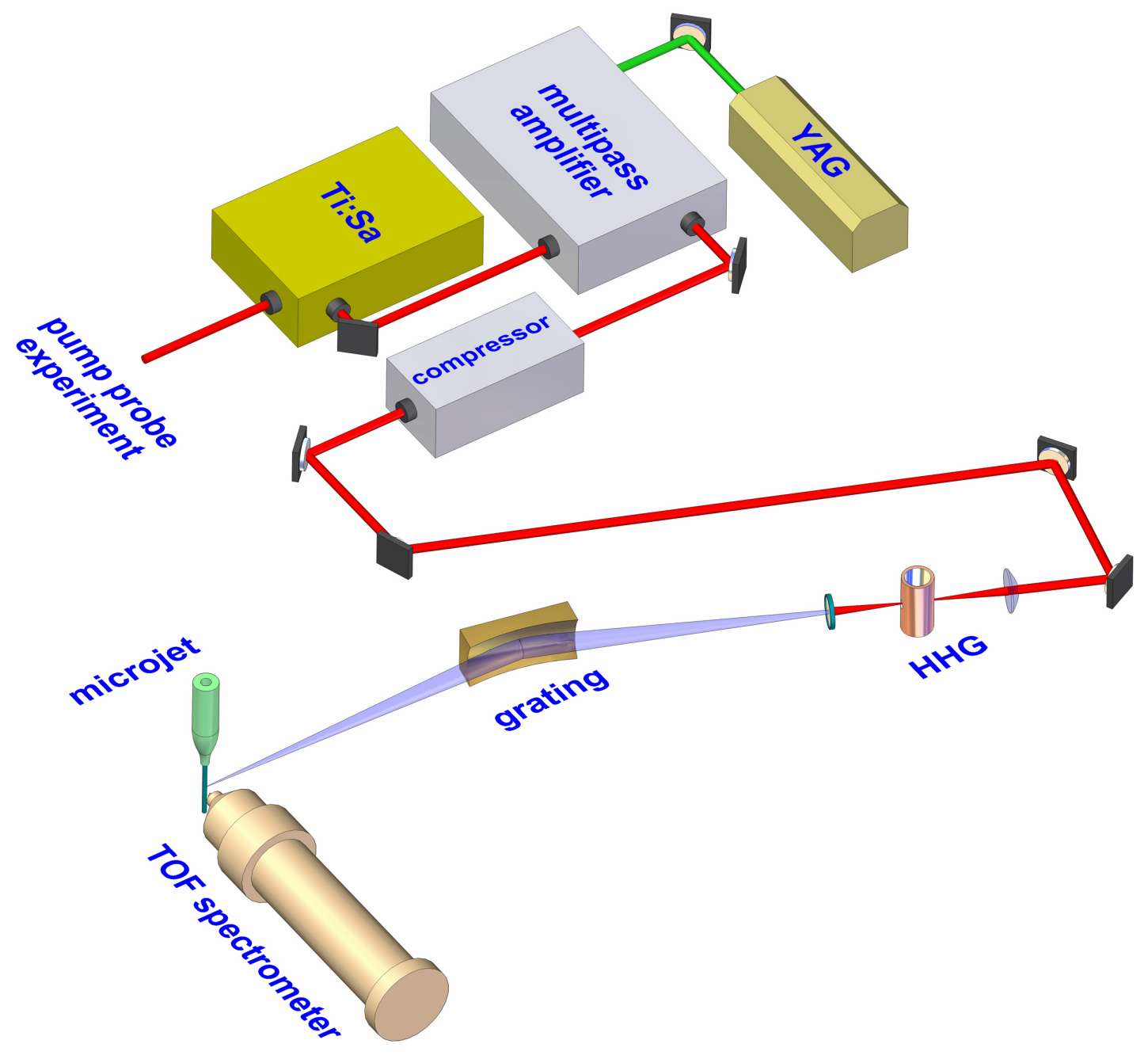

Figure 4.1: The complete laser beam line for the PES experiment. The essential units: Ti:Sa system, multipass amplifier, compressor, generation and selection of EUV light via HHG and toroidal grating.

pulsed Nd:YLF laser (Evolution ${ }^{\mathrm{TM}}, 8 \mathrm{~W}, 527 \mathrm{~nm}, 50 \mathrm{~ns}$ ) pumps this crystal at a repetition rate of $1 \mathrm{kHz}$ and induces a population inversion. A Pockels cell sends the stretched pulse into the resonator with a $100 \mathrm{~ns}$ time delay. This pulse will be amplified by passing the crystal and inducing stimulated emission therein. After about 20 resonator cycles, another Pockels cell releases the pulse from the regenerative amplifier. 
Multi-pass amplifier Usually, the intensified pulse from the regenerative amplifier in a Hurricane ${ }^{\mathrm{TM}}$ system passes a compressor unit (reverse to the stretcher unit) and the final output would be a $100 \mathrm{fs}$ short laser pulse with a pulse energy of $1.0 \mathrm{~mJ}\left(1 \mathrm{kHz}, \lambda_{\text {central }}=800 \mathrm{~nm}\right)$. For the present experiment, we use only a small part of the output from the regenerative amplifier $(0.2 \mathrm{~mJ})$ and guide it to a home-made external multi-pass amplification unit, which is shown in Fig.4.2. Its essential component is an Ti:Sa crystal cooled to $18^{\circ} \mathrm{C}$ and pumped by a frequency doubled Nd:YAG laser (Clark-MXR, ORC-1000, 532 nm, 1 kHz, 8 W). Every pulse from the Hurricane ${ }^{\mathrm{TM}}$ system passes the crystal three times and is amplified to $2 \mathrm{~mJ}$. A delay generator (Stanford Research Systems) correlates the timing of the Hurricane $^{\mathrm{TM}}$ and pump pulse in the crystal. A two-grating unit compresses the amplified pulses after the three passes to $110 \mathrm{fs}$ short pulses of $1.2 \mathrm{~mJ}$.

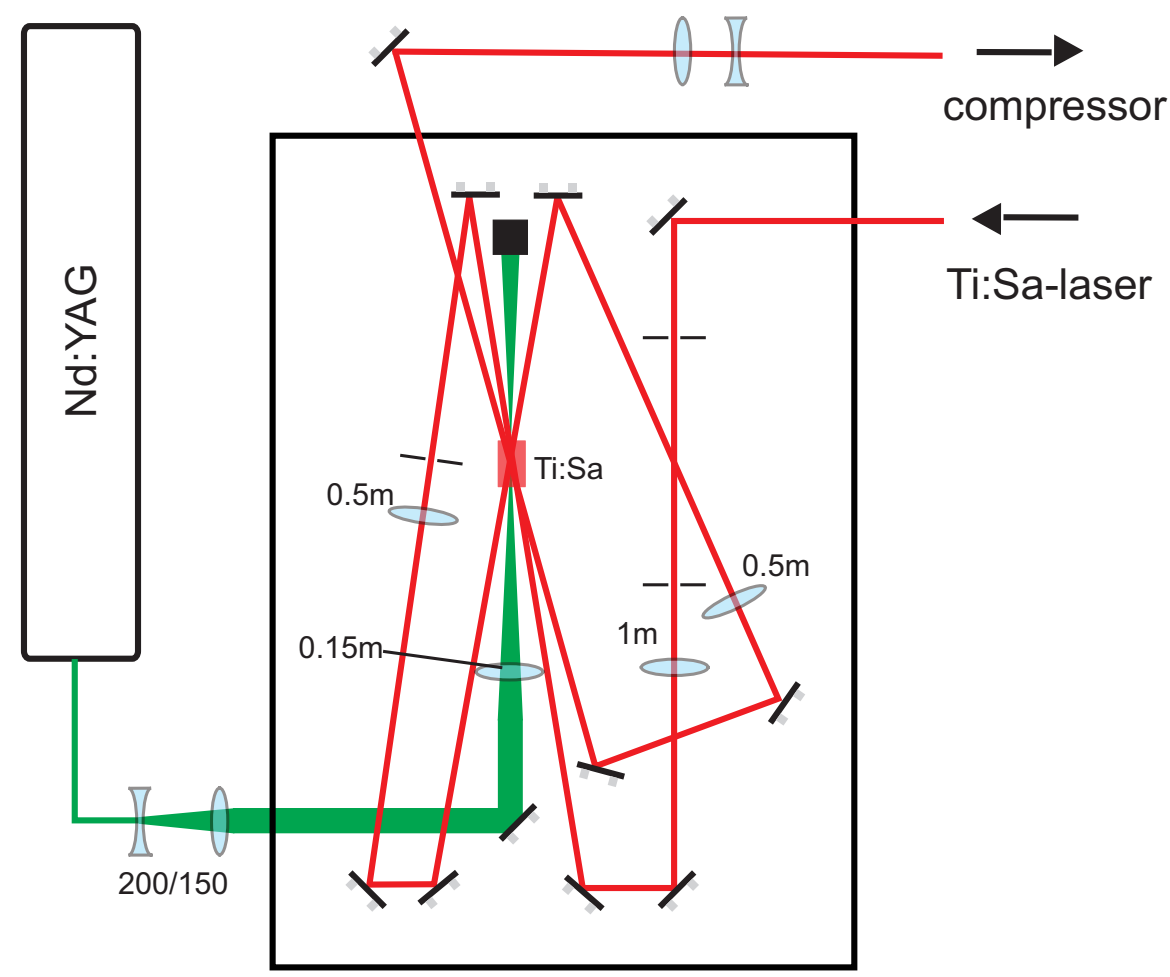

Figure 4.2: Sketch of the multi-pass amplification unit. 


\subsection{Light polarization tuning}

For the investigation of angular distribution of photoelectron emission, measurements have to be made, at different angles $\theta$ between the photoelectron detecting direction and the polarization vector of the ionizing radiation (see 2.4). For this purpose, the polarization vector of the EUV light will be tuned, since the photoelectron analyzer, a time-of-flight photoelectron spectrometer (see 4.6) is fixed perpendicular to the light propagation direction and in the plane parallel to the optical table.

A direct tuning of EUV light polarization with any conventional optical elements is almost impossible because of the special optical requirements of this radiation. However, we can effortlessly rotate the polarization vector of the $800 \mathrm{~nm}$ fundamental light with a $\lambda / 2$ plate, also called polarization rotator. The $\lambda / 2$ plate is positioned in the beam path of the fundamental radiation into the HHG chamber and in front of the iris that tailors the beam diameter of the $800 \mathrm{~nm}$ light. By rotating the optical axis of the $\lambda / 2$ plate in any angle with reference to the initial polarization vector of the fundamental light, all of the required $\theta$ can be attained. The tuning of the $\lambda / 2$ plate works as follows: its optical axis has to be turned by $\alpha / 2$ if a rotation of the polarization vector by $\alpha$ is required. Notice that this procedure is based on the fact that the high harmonics possess (nearly) the same polarization as the generating radiation (see subsection 3.2.3).

The initial polarization plane of the $800 \mathrm{~nm}$ fundamental light is parallel to that of the optical table, on which the HHG chamber is mounted. This polarization is commonly defined as $\boldsymbol{p}$-polarization while the polarization vertical to it is called $s$-polarization. This nomenclature is also used in this work. At $p$-polarization, the angle $\theta$ between the photoelectron detecting direction and the polarization vector of the ionizing radiation is $0^{\circ}$, while $\theta=90^{\circ}$ for $s$-polarization. In addition, there is another relevant angle $\theta=54.7^{\circ}$, also called the magic angle, which plays an important role in the determination of the angular distribution of photoionization.

It must be noted that the focusing grating used here has different reflection properties for EUV radiation with various polarization vectors. This is related to the special optical properties of the grating (see subsection 3.2.2). Hence, the intensity of the ionizing light at the target position is influenced by its polarization 
direction. By means of the reflection curves of the grating given by the manufacturer, the intensity of $p$-polarized light is estimated to be about four times higher than that of $s$-polarized light.

\subsection{The vacuum apparatus}

Not only the progression of EUV radiation but also the detection of photoelectrons require (ultra) high vacuum conditions because unwanted particles can absorb the EUV radiation or interfere with the photoelectrons. To avoid absorption of EUV radiation, vacuum conditions of $<10^{-3}$ mbar are needed, and $<10^{-5}$ mbar for the proper detection of electrons. Additionally, the MCP-detector in the TOF-spectrometer and the CCD-camera can only be used below $10^{-6}$ mbar. The schematic construction of the complete vacuum apparatus is shown in Fig.4.3. It consists of four connected chambers which are evacuated individually.

In the chamber with the high harmonic generation unit (HHG chamber), a high efficient vacuum system is required, since the Ar-gas effuses permanently into the chamber through two holes in the capillary. For this reason, we use a roots and a rotary vane pump. The pressure in the HHG chamber can be kept at a few $10^{-2}$ mbar during operation.

The grating chamber is pumped by a scroll and a turbo-molecular pump, and is separated from the HHG chamber by a $150 \mathrm{~nm}$ thick Al-filter. This guarantees the pressure required $\left(10^{-6}\right.$ mbar $)$ for the grating.

From the top of the main chamber, the liquid micro-jet or the cluster nozzle holder can be inserted. In order to ensure low vapor pressure conditions $\left(10^{-4} \mathrm{mbar}\right)$, a turbo-molecular pump and a roots pump are used. Two cold traps are integrated in this chamber: the first one sits directly below the target position and can freeze out the liquid beam. The other one is placed near the turbomolecular pump.

The CCD-camera for monitoring the EUV radiation is attached to the main chamber on the axis of the incoming light. A small turbo-molecular, a rotary vane pump and a valve with Al-filter in the center protect the CCD against damage caused by adsorption of any particles. 


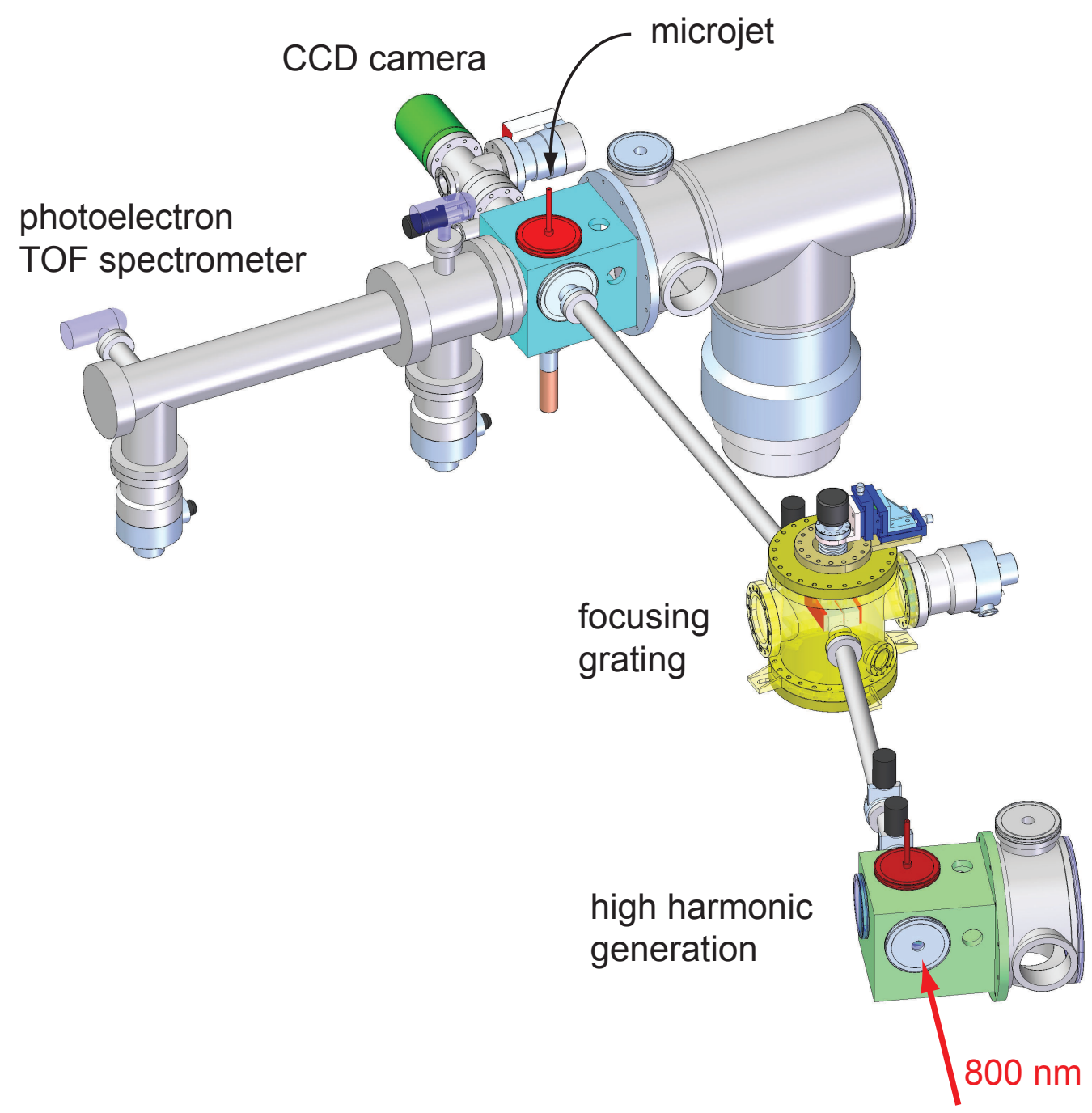

Figure 4.3: Detailed picture of the vacuum setup composed of our table top beamline (high harmonic generation chamber and grating chamber), the main chamber containing the liquid micro-jet and a time-of-flight photoelectron spectrometer.

Perpendicular to the propagation axis of the EUV light, the time-of-flight photoelectron spectrometer (see subsection 4.6) is connected to the main chamber. A titanium skimmer (aperture $\varnothing=250 \mu \mathrm{m}$ ) separates two different vacuum ranges: $>10^{-5}$ mbar in the main chamber and $<10^{-6}$ mbar in the spectrometer, which is kept at this pressure by two additional turbo-molecular pumps. 


\subsection{Liquid micro-jet}

All presented photoelectron spectra of liquid and gas phase in this work were obtained using a liquid micro- jet sample. The micro-jet technique enables us to maintain a clean liquid surface under high vacuum conditions. The design requires the generation of a liquid jet with a diameter $d_{j e t}$ which is comparable to or smaller than the mean free path $\lambda_{m o l}$ of the respective molecules in the medium [29]. Only then it conforms to the well known Knudsen condition

$$
\lambda_{m o l} \leq d_{j e t} .
$$

The mean free path can be estimated with

$$
\lambda_{m o l}[\mathrm{~cm}]=\frac{c}{p_{v}[\text { Torr }]}
$$

given by [70].

For liquid water at $273 \mathrm{~K}\left(p_{v}=4.58\right.$ Torr and $c=5.3 \cdot 10^{-3}$ Torr $\left.\cdot \mathrm{cm}\right)$, a jet diameter of at most $11.5 \mu \mathrm{m}$ is needed to obtain a liquid surface from which the molecules evaporate nearly collision-less into vacuum. Thus, the density of gas phase molecules decreases rapidly with increasing distance from the liquid jet. This allows photoelectrons outgoing from the liquid to survive the thin and sparse gas phase above the liquid surface almost without collisions and to be analyzed via the liquid phase PES approach.

Technical details In this experiment we generate the micro-jet by using a HPLC pump (Economy 2/ED, Techlab Instruments) to press the liquid through a quartz glass nozzle. The nozzle has an opening diameters of $16 \mu \mathrm{m}$ - resulting in liquid cylindrical filaments with diameters of $12 \mu \mathrm{m}$. With a flux rate of $0.4 \mathrm{ml} / \mathrm{min}$ the liquid jet remains stable over a distance of about $2-3 \mathrm{~mm}$ from the nozzle tip and then breaks up into a stream of droplets which will be frozen out in a trap cooled with liquid nitrogen. An additional cooling trap and a powerful turbo molecular pump (1600 l/s, Pfeiffer) are used to keep the ambient pressure in the main chamber below $2 \cdot 10^{-5}$ mbar. As a means to eliminate charging effects on the liquid jet, a negligible amount of salt must be added to the probed liquid. A $0.005 \mathrm{~mol} / l \mathrm{NaCl}$ solution prepared with doubly distilled water was utilized in this work. 


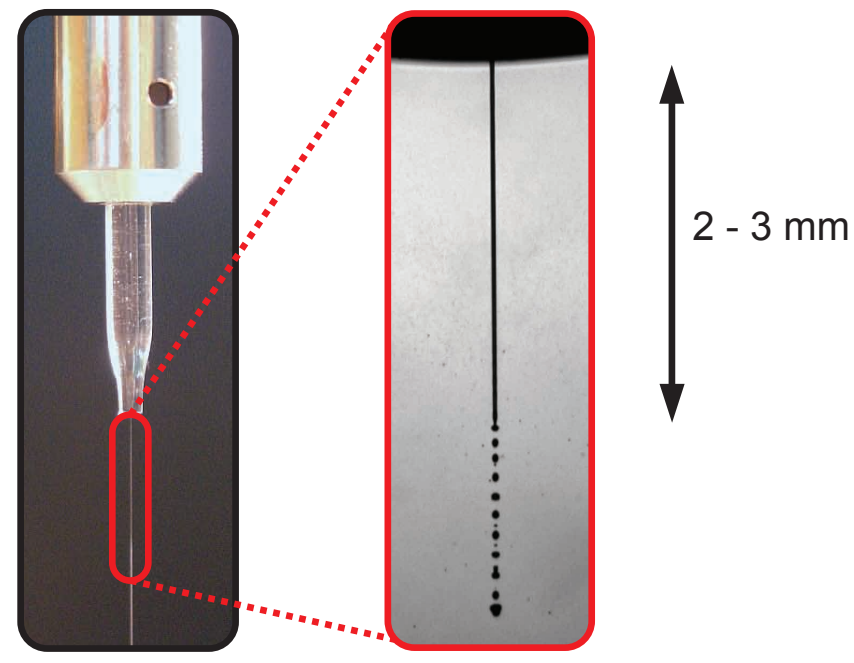

Figure 4.4: Picture of the liquid micro-jet.

Surface temperature We are also interested in the temperature on the surface of the liquid micro-jet. During expansion through the quartz nozzle into the vacuum, evaporative cooling occurs which cools down the liquid. Faubel et al. determined the temperature of the liquid jet surface by investigating the kinetic energy distribution of the evaporating molecules [71]. For a $10 \mu \mathrm{m}$ diameter jet and an initial temperature of $293 \mathrm{~K}$ they found a surface temperature of about $280 \mathrm{~K}$ for a mixture of $20 \%$ acetic acid and $80 \%$ water. In our experiments we generate liquid jets with larger diameters. It is expectable that the water micro-jet surface temperature under our vacuum condition is between $273-278 \mathrm{~K}$ [72].

Surface sensibility Another important aspect of the PES experiment on a liquid micro-jet is the limited escape depth of photoelectrons. A photoelectron ejected from liquid bulk travels through some water layers lying above its origin and does not always retain its kinetic energy until it leaves the surface. The reason for it are the inelastic scattering events. Only direct or elastically scattered photoelectrons contribute to the photoelectron spectrum. The so-called inelastic mean free path (IMFP) of an electron determines if it can reach the vacuum without losing kinetic energy. The IMFP is largely determined by the material-specific dielectric permittivity function and shows for most materials a similar dependence on the 
electron kinetic energy, characterized by a minimum of about $5-10 \AA$ for kinetic energies between $50-100 \mathrm{eV}$, with a steep increase towards the low-energy side, and a steady but slower increase in the higher energy range [24].

Another quantity also mentioned in this context is the effective electron attenuation length (EAL) [74]. It defines the shortest distance from a starting point $S$ to an arrival point $A$, at which the initial electron signal is reduced to 1 /e. For weak elastic scattering, as in the case of liquid water, EAL and IMFP are almost equal. Although, if elastic scattering is efficient, IMFP can be significantly larger than EAL. Considering that our liquid micro-jet temperature is near the freezing point of water, we determine the EAL of the photoelectrons from liquid phase with the data of amorphous ice from [73]. For electrons with $20-30 \mathrm{eV}$ kinetic energies, EAL is evaluated to be $30-40 \AA$. However, the EAL value for a cylindrical jet surface is even smaller by a factor of $2 / \pi$ than that for a thin planar film, when photoelectrons are observed at $90^{\circ}$ with respect to the photon propagation direction [75]. As a result, the electron attenuation length in our experiment is about $20-30 \AA$ for photoelectrons outgoing from the three outer orbitals of liquid water. This means, the average subsurface probing depth in our liquid jet experiments is about $7-10$ monolayers of water molecules assuming that a monolayer in liquid water amounts to $3 \AA$. Based on this estimate, we expect that the (quasi-)bulk properties of liquid water are investigated in our studies.

\subsection{Cluster source}

Technical design In order to obtain small neutral water clusters, we constructed a cluster source based on the principle of supersonic expansion through a conical nozzle. Figure 4.5 shows a sketch of the cluster source. The reservoir can be filled with $20 \mathrm{~m} l$ liquid and is temperated by a thermostat via a heating fluid, a mixture of glycol and water. The reservoir is connected to the nozzle holder unit by a $10 \mathrm{~cm}$ short steel capillary $(\varnothing=3 \mathrm{~mm})$. The nozzle holder has an interior pipe of $5 \mathrm{~mm}$ diameter which is enclosed by a outer tube $(\varnothing=12 \mathrm{~mm})$. A second thermostat circulates heating solution through this tube and keeps the nozzle temperature constant. The conical nozzle is mounted to the end of the holder with a copper seal ring and fixed with six screws. In this experiment we use a conical nozzle with 
a diameter $d=160 \mu \mathrm{m}$ and a length of $2 \mathrm{~mm}$. The half opening angle of the nozzle is $\alpha=12.5^{\circ}$. Additionally, we monitor the reservoir and nozzle temperature with two separate thermocouples. The nozzle temperature must always be higher than that of the reservoir so that the water vapor does not freeze and block the opening of the nozzle.

The estimation of mean cluster size To estimate the mean size $\langle n\rangle$ of clusters generated via adiabatic expansion, the scaling laws method is commonly applied, which is thermodynamically based, although empirical [76, 77]. Related to references [78] and [13], the following equation can be assumed:

$$
\langle n\rangle=D\left(\frac{p_{0} d_{e q}^{q}\left(\frac{T_{n}}{T_{c} r_{c}}\right)^{q-3}}{1000 k_{B} T_{n}}\right)^{a}
$$

to estimate the mean size of clusters generated by our cluster source described above. Here, $a, q$ and $D$ are empirical parameters, $T_{c}$ and $r_{c}$ are the characteristic temperature and radius, and $k_{B}$ is the the Boltzmann constant. The stagnation

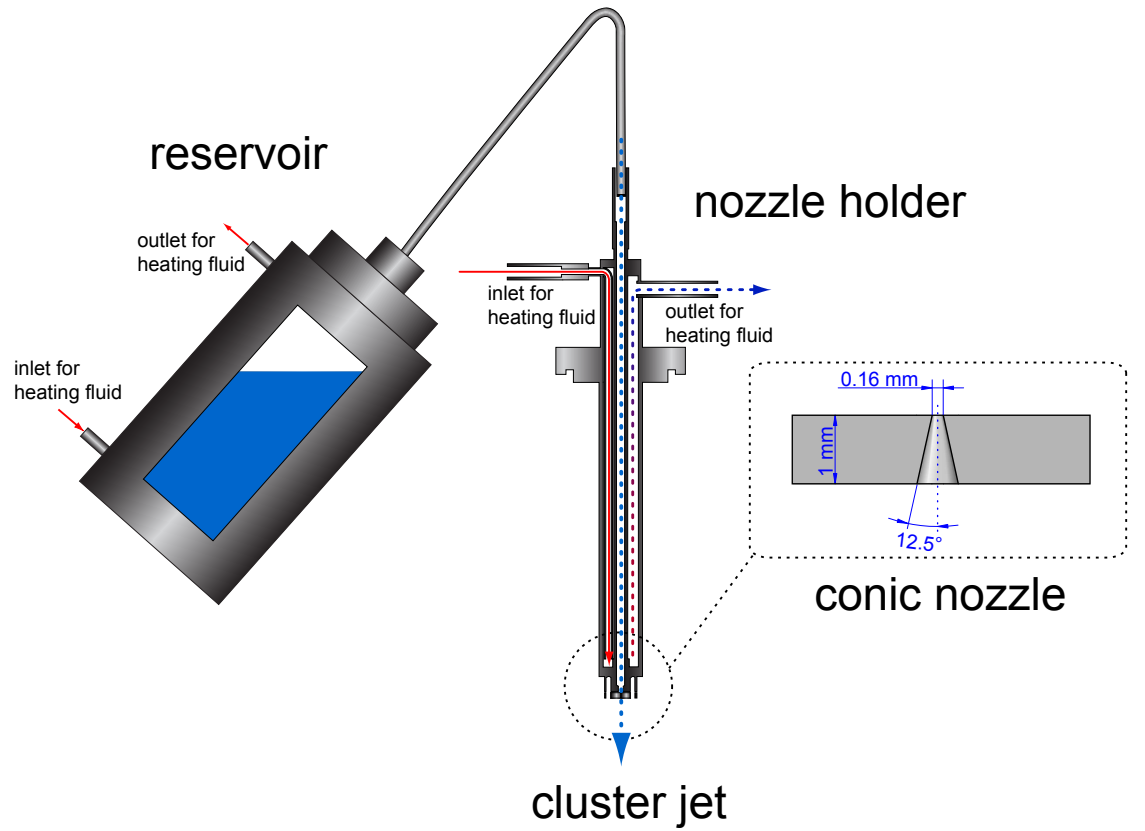

Figure 4.5: Sketch of the cluster source. 
pressure of the expansion $p_{0}$ derives from the vapor pressure at the given temperature $T_{r}$ and therefore is a function of the latter $p_{0}=f\left(T_{r}\right) . d_{e q}$ denotes the equivalent diameter of a certain nozzle which is defined as

$$
d_{e q} \doteq d / \tan \alpha
$$

and for the above described nozzle $d_{e q}=721 \mu \mathrm{m}$. Both, the nozzle shape $(d, \alpha)$ and $T_{r}$ are crucial for the cluster size while $T_{n}$ is less decisive. It must be noted that the estimation of mean cluster size using the scaling laws method is limited for very small clusters $(n<10)$, due to the large deviation [78].

\subsection{TOF-spectrometer}

To detect and analyze photoelectrons, a time-of-flight photoelectron spectrometer (Kaesdorf) and a multi channel plate (MCP) detector (Burle) are applied in our experiment (see Fig.4.6). TOF detectors provide major advantages for measurements in which a particularly high resolution is not necessary. The most important advantage is, that the entire spectrum is collected simultaneously for each high harmonic laser pulse, which significantly shortens the overall acquisition time per spectrum.

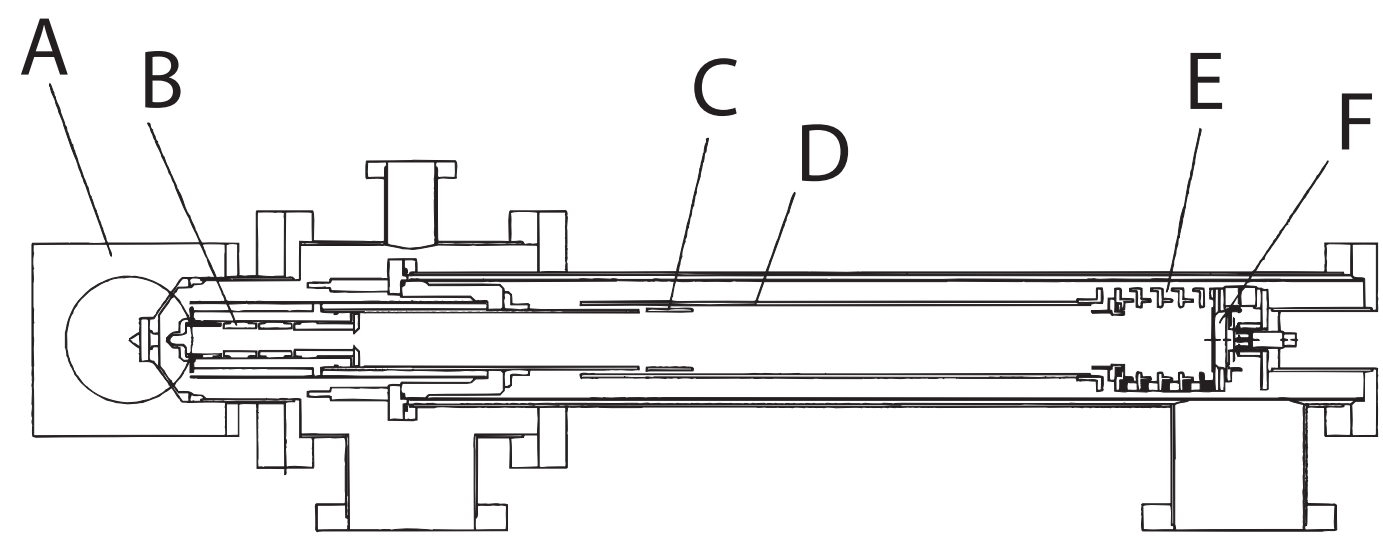

Figure 4.6: Sketch of the TOF photoelectron spectrometer: A) main chamber, B) braking optics, C) distract unit, D) drift tube, E) acceleration range, F) MCP-detector. 
In this experiment, the liquid micro jet is positioned $200-500 \mu \mathrm{m}$ in front of the titanium skimmer (aperture $\varnothing=250 \mu \mathrm{m}$ ) which defines, together with another skimmer (aperture $\varnothing=2 \mathrm{~mm}$ ) within the spectrometer, the direction of detected photoelectrons. A $\mu$-metal shielding excludes any magnetic fields from the inside of the spectrometer while six Helmholtz coils in cubic arrangement ensure that the region between the liquid jet and the spectrometer entrance is field-free. A photoelectron must travel a flight length of $78 \mathrm{~cm}$ till being detected. Its time of flight $t_{f}$ depends on its initial kinetic energy $E_{\text {kin }}$ and can be recorded by a MCP and analyzed with an A/D-converter (9353 100 ps Time Digitizer, Ortec). The A/D-converter is triggered by the signal of a fast photodiode which picks up the fundamental $800 \mathrm{~nm}$ light pulse. Steering optics in the drift tube focus the electrons in order that they can fly straightly towards the detector. An acceleration unit $(280 \mathrm{~V})$ in front of the detector enhances their detection probability. Two turbo molecular pumps (Pfeiffer), together with a membrane pump, ensure high vacuum conditions with a pressure of $\leq 10^{-6}$ mbar in the spectrometer. To transform photoelectron spectra from the time-of-flight domain into the binding energy domain we determined the following calibration function:

$$
E_{\text {bind }}=h \nu-\left(2.528 \cdot 10^{6} \cdot t_{f}^{b}+\text { offset }\right)
$$

Notice the detailed description of the calibration function in [68]. The parameters $b$ $(\approx-2.058)$ and offset can be individually adjusted for each series of measurements. The TOF-spectra have a maximum resolution of $0.1 \mathrm{~ns}$. Accordingly, the resulting energy resolution of the transformed spectra is $0.25 \mathrm{eV}$. In Fig.4.7 a gas phase spectrum of water is given as an example for the successful transformation using this calibration function. The binding energies for the three valence orbitals refer to the corresponding values in [25]. 


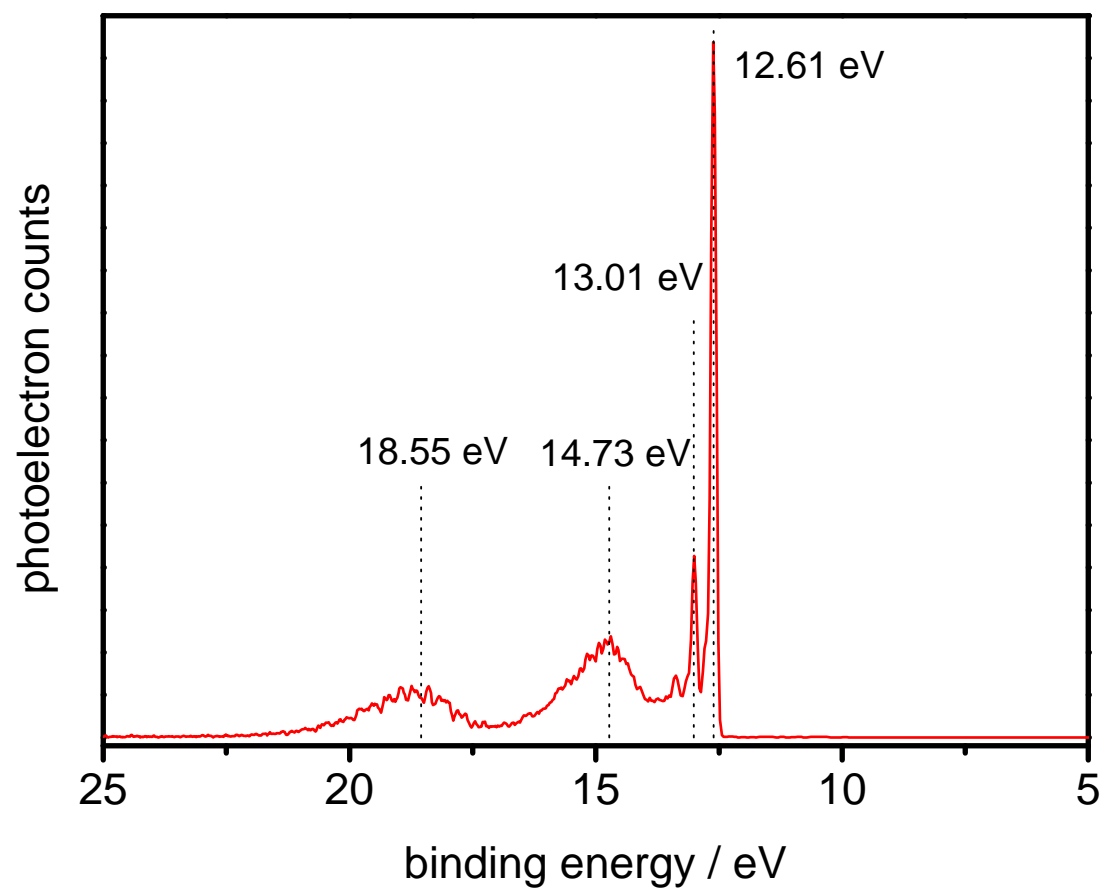

Figure 4.7: A transformed spectrum of water vapor. The binding energies for the three valence orbitals refer to the corresponding values in [25]. The parameters of the calibration function are: $b=2.058$ and offset $=2.1$. 


\section{Chapter 5}

\section{The angular distribution of photoemission from water molecules, clusters and the bulk}

\subsection{Introduction}

Water is the most abundant substance on our planet and further the principal constituent of all living organisms. Accordingly, chemical reactions in liquid water are essential for many important processes in nature. Understanding the properties of water on a molecular basis is crucial for many fields of research ranging from biochemistry and cellular biology to atmospheric chemistry and planetary physics. Hence, water has probably been the most extensively investigated compound.

Nowadays, the single water molecule $\mathrm{H}_{2} \mathrm{O}$ is very well characterized. A great deal of experimental and theoretical research revealed its fundamental properties, such as geometry, binding and dipole moment. Diverse spectroscopic methods allowed important insights into vibrations, rotations and electronic structure of the $\mathrm{H}_{2} \mathrm{O}$ molecule.

The symmetry group of the $\mathrm{H}_{2} \mathrm{O}$ molecule is $C_{2 v}$. There are two mirror planes which are perpendicular to each other. The two $\mathrm{O}-\mathrm{H}$ bonds were found to be $0.9576 \AA$ long and the angle between them amounts to $104.5^{\circ}$ [79]. The majority of the electron density in the $\mathrm{H}_{2} \mathrm{O}$ molecule is localized around the oxygen, whereby the average electron density around the oxygen atom is about 10 times that around the hydrogen atoms. The binding relation of the three atoms can be illustrated 
via linear combinations of the electronic wave-function of each atom within the LCAO-MO-approximation. Because of the different electronegativity of oxygen (3.44) and hydrogen (2.20), the $\mathrm{O}-\mathrm{H}$ bond is polar and a water molecules has a dipole moment of $\mu=1.86 \mathrm{D}$ [80]. The nature of the $\mathrm{O}-\mathrm{H}$ bond is mainly covalent but to a partial extend ionic. In the ground state, the $\mathrm{H}_{2} \mathrm{O}$ molecule exhibits the following electron configuration:

$$
\left(1 a_{1}\right)^{2}\left(2 a_{1}\right)^{2}\left(1 b_{2}\right)^{2}\left(3 a_{1}\right)^{2}\left(1 b_{1}\right)^{2} \text {. }
$$

Here, $1 b_{1}, 3 a_{1}$ and $1 b_{2}$ are the three outer occupied molecular orbitals (MO) and the MO-diagram is shown in Fig. 5.1. The $1 b_{1}$ orbital is the highest occupied molecular orbital (HOMO) and its nodal plane lies in the plane including all atoms. The $3 a_{1}$ and $1 b_{2}$ orbitals are both symmetric with respect to the molecular plane. The $3 a_{1}$ orbital has a nodal plane perpendicular to both symmetry planes of the molecule, while $1 b_{2}$ is antisymmetric with respect to the bonds. The $1 b_{1}$ orbital has non-bonding character and hence is called lone pair orbital. The $3 a_{1}$ and $1 b_{2}$ orbitals are responsible for the $\mathrm{O}-\mathrm{H}$ bond, whereby the $1 b_{2}$ contributes the most. The $1 b_{2}$ orbital contains bonding combinations of $\mathrm{O} 2 p$ and $\mathrm{H} 1 s$, while the $3 a_{1}$ orbital possesses essentially $\mathrm{O} 2 p$ non-bonding character with a small share of $\mathrm{H} 1 s$. The $4 a_{1}$ orbital is the lowest unoccupied molecular orbital (LUMO). In water aggregates, liquid water and ice, it "accepts" lone pair electrons from the $1 b_{1}$ orbitals of neighboring water molecules - within Pauling's definition of hydrogen bonding.

There are three normal vibrations in $\mathrm{H}_{2} \mathrm{O}$ molecule: the symmetric stretching vibration $v_{1}$, the symmetric bending vibration $v_{2}$ and the asymmetric stretching vibration $v_{3}$. From a highly resolved $\mathrm{PE}$ spectrum of gaseous water, information about these three vibrations in the cation $\mathrm{H}_{2} \mathrm{O}^{+}$can be obtained. The first highly resolved PE spectrum of free water molecule using the $\mathrm{He}(\mathrm{I})$ line was published by Brundle and Turner in 1968 [25]. This spectrum exhibits three distinct bands which are attributed to the outer three valence orbitals of water molecule $1 b_{1}$, $3 a_{1}$ and $1 b_{2}$. Their vertical ionization potential (from the strongest peaks) are $12.61 \mathrm{eV}, 14.73 \mathrm{eV}$ and $18.55 \mathrm{eV}$. The vibrational fine structures of the bands are very different, according to the bonding character of the respective orbitals. 


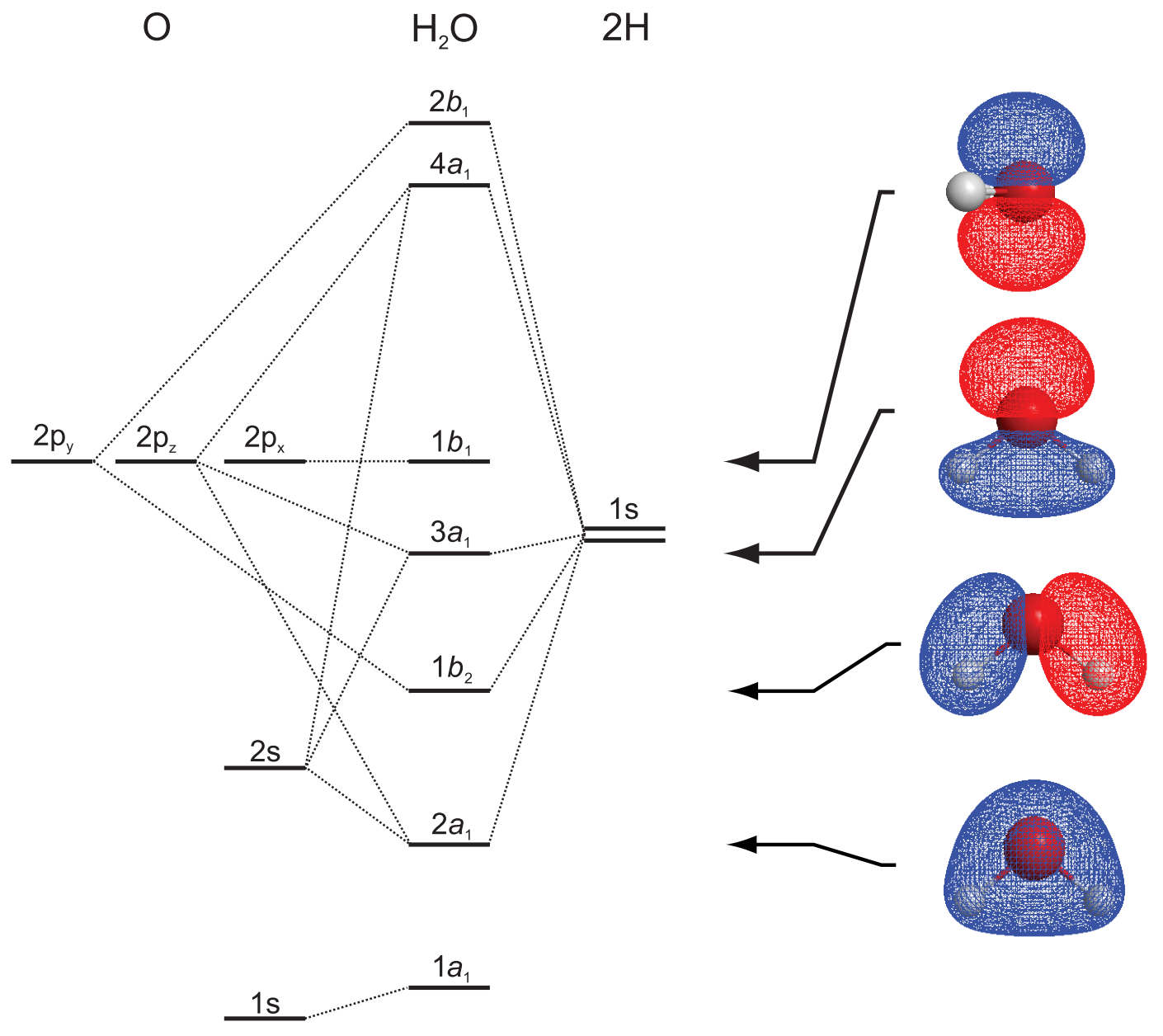

Figure 5.1: The MO diagram of $\mathrm{H}_{2} \mathrm{O}$ molecule. The four outmost valence orbitals are shown (geometry optimized and orbitals calculated with basis set aug-cc-pVQZ at MP2 level using GAMESS [81]). 
Knowledge about water in the gas phase paved the road for understanding the unique properties of liquid water, which is a particularly challenging task. The reason is that water molecules build hydrogen bond networks, when going from the gas into the liquid phase. This network is responsible for probably all the unique properties of liquid water, e.g. the density anomaly of water at $4^{\circ} \mathrm{C}$, the strong polarizability and the significant increase of the dipole moment from $1.86 \mathrm{D}$ in the gas to $3 \mathrm{D}$ in the liquid phase [2].

The hydrogen bond (also H-bond) in water is an attractive interaction between a hydrogen atom of a water molecule (called $\mathrm{H}$-donor) and a lone pair of electrons located at the oxygen atom of another molecule (H-acceptor). One water molecule can build up to four H-bonds with four neighboring water molecules, which is the case in crystalline ice. The average coordination number in liquid water is still subject to debate. Molecular-dynamics simulations of liquid water at ambient conditions indicated that the average number of hydrogen bonds per molecule is between 3 and 4 [82, 83], while a value of $2.4-2.6$ was found by an X-ray absorption study [19]. The strength of H-bonds in water is thermodynamically estimated to be about $23.3 \mathrm{~kJ} / \mathrm{mol}$ [84], which is intermediate between covalent or ionic and van der Waals bonding. This value of the bonding strength is equal to the energy which is required for separating the H-bonded molecules completely and promoting them into gas phase. There is also another definition for the strength of H-bonds in water. Smith et al. determined a value of $0.065 \mathrm{eV}$ for the energy required to break one hydrogen bond in liquid water while leaving the molecules essentially in the same position [20]. The H-bonds in water are strong enough to create stable intermolecular structures but also sufficiently weak to be easily broken during the dynamic rearrangement in liquid water. As generally accepted, there are a number of contributions to H-bonding: electrostatic attraction, polarization and dispersion interaction. The first one preponderates and the strongest electrostatic attraction in this case is dipole-dipole interaction. Since ten years, another supposed contribution to H-bonding has attracted more and more attention - charge transfer mechanism, which can also be described as a partially covalent nature of hydrogen bonds [3-6]. 
There have been a lot of experimental and theoretical investigations which were concerned with understanding the nature of H-bonds. A short introduction to the topic is presented here with a focus on how the presence of H-bonds affects the electronic structure of water molecules in different aggregate states. These states can be liquid, clusters or solid. Observation at the level of molecular orbitals is essential for revealing the real nature of the H-bonds of water. The following text briefly reviews the reported investigations, which have concentrated on studying the electronic structure of H-bonded water systems.

Due to the limited experimental methods to prepare a clean liquid surface in vacuum for the investigation using electron spectroscopic techniques, it has been quite difficult to examine the electronic structure of liquids. The experimental studies of liquid water have not been really possible until several innovative sample handling methods were devised for this purpose (see subsection 2.3.3). The relevant studies have been performed using valence photoelectron spectroscopy [10 12], Xray emission spectroscopy (XES) [14, 15, 17, 18] or X-ray absorption spectroscopy (XAS) [19, 20]. In addition, the Auger spectrum of liquid water has been calculated by a Green's function method [85]. Several theoretical calculations allowed further insight into what happens in the phase transition from the gas to the liquid phase [86 88]. In reference [11], Winter et al. presented full-range valence PE spectra of liquid water measured at the BESSY synchrotron beamline with photon energies up to $120 \mathrm{eV}$. Their study is comparable with ours, since that experiment was also carried out using the liquid micro-jet technique (see subsection 4.4. They have extensively analyzed the different PE spectral features of liquid water by comparison with those of gaseous water. Core level spectroscopic techniques such as X-ray absorption and X-ray photoemission spectroscopy were applied to search for the relationship between the electronic and geometric structure of liquid water, since the different H-bonding configurations were expected to show a variation in the local water molecular orbital structure [17, 19]. For instance, a specific configuration in bulk water was identified by Myneni et al. which is related to a pre-edge feature in the XAS spectra of liquid water [19]. This configuration was suggested to be a local network structure with a broken H-bond on the H-donating site of one water molecule. This assignment was confirmed by another X-ray emission study [17], whereby resonantly excited XE spectra were 
measured. Moreover, in that work a strong involvement of the $3 a_{1}$ orbitals in the H-bonding was found. The accompanying calculation showed that the major influence of the hydrogen bonding on the local electronic structure is due to the first coordination shell.

The investigation of water clusters offers an unique approach to study the transition from the gas to the liquid or solid phase. By changing the average size of the clusters, information about the gradual evolution of the electronic properties related to the H-bonding effects can be obtained. Many experimental studies have been carried out using valence, core-level or Auger photoelectron spectroscopic approaches [13, 21, 89]. There have also been a good deal of calculations based on various approaches for different sized clusters [90 93], which complement the quantum mechanical picture of the electronic structure of water molecules in H-bonded small aggregates. The obtained information from these cluster investigations offers new constraints and a different perspective on the bulk, which might be effectively applied to bulk properties. Core and valence photoemission spectra in 21] showed a weak but gradual change with cluster size. The upwards shift from the molecule over small to large clusters was associated with a gradual increase in the average coordination number of the cluster structures. Hergenhahn and co-workers reported recently their PES studies on water clusters using synchrotron and ab initio spectra calculations [13]. Shifts of vertical ionization energies towards lower values with increasing cluster size were shown. They observed that the ionization energies of clusters decrease linearly with inverse cluster radius and asymptotically approach the value of liquid water. Moreover, they suggested that the measurement of electron ionization energies can be used as an alternative method to characterize water cluster sizes (see section 4.5). One of the barely touched fields in PES on water clusters is the electronic structure of very smallsized clusters $\left(\mathrm{H}_{2} \mathrm{O}\right)_{n}$ with $n<10$. This lack is due to the fact that the generation and selection of such small clusters is very difficult to realize in practice and the combination with photoelectron spectroscopy complicates the initial situation of such an experiment even more. Up to now, information on the electronic structure of small clusters comes from theoretical calculations exclusively.

Analyzing the effects of H-bond networks on the electronic structure in solid water is also one of the most important approaches to examine the nature of 
H-bonds. Because the geometric structure of crystalline ice is firmly defined, effects of H-bonds on the orbital picture can be obtained without considering influence from molecular dynamics in water. Experimental investigations concerning the electronic structure of ice have been carried out using photoelectron spectroscopy [6, 7, 94 96], X-ray emission spectroscopy[16, 17] or X-ray absorption spectroscopy [6]. These experiments were often combined with theoretical simulations. The valence PE spectra of water vapor and ice have been already reported in the 1960s by Kai Siegbahn and co-workers [97]. Another early PE study on ice [16] showed major electronic structure changes relative to the gasphase, including binding energy shifts and band broadening. They reported a rather unperturbed $1 b_{1}$ orbital and a delocalized $3 a_{1}$ in ice. The conclusion on a delocalized $3 a_{1}$ orbital was based on the observation that the corresponding bands exhibit a double peak structure, which was associated with strong orbital overlap between neighboring $3 a_{1}$ orbitals. In a PE and XAS study in combination with a density functional theory (DFT) calculation, a novel hypothesis was presented - the hydrogen bond involves charge donation from the lone pair of one water molecule to the $\mathrm{OH}$ anti-bonding orbitals on neighboring molecules. In addition, an internal rehybridization of the valence orbitals was proposed, which results in a lower electronic repulsion between water molecules [6]. This is in line with the charge transfer picture of hydrogen bonding that is derived from a natural bond orbital analysis [96].

One of the common points of the above-mentioned studies in different phases is that the spectral differences between water vapor and H-bonded water aggregate states were discussed. In all cases, the spectral differences between free water molecules and another H-bonded aggregate state suggested that the electronic structure of water molecules undergo significant changes. These variations were consistently interpreted as evidence for the changes in electronic structure resulting from the significant involvement in H-bonding. Especially, the dissimilarity of the spectral variations of valence orbitals was often interpreted in terms of their different involvement in the intermolecular interactions. At this point there have been a few rounds of intensive disputation about which valence orbital is mostly involved in the H-bonding. The vast majority estimated that the involvement of 
$3 a_{1}$ orbital is the strongest in H-bonding [6, 16, 17], while there was the contrastive supposition of the intensive effect of the H-bonding on the $1 b_{2}$ orbital [11].

Another common point of these works is the similar general motivation - to interpret the nature of the hydrogen bond on the basis of changes in the electronic structure. Therefor a microscopical picture is needed, which can appropriately describe the molecular origin of the interaction between water molecules in the liquid. The H-bond in most theoretical studies has been considered as either a pure electrostatic interaction, including internal polarization from the field of the neighboring water molecules, or as involving charge transfer between the molecules, sometimes interpreted as covalent bonding [6]. Mostly this chemical interaction is regarded as a charge redistribution within the molecule induced by the field of neighboring molecules [96, 98,100]. Although it has been broadly accepted and used as basis, to simulate many classical molecular dynamics force field descriptions of liquid water [100 103], is this electrostatic picture of hydrogen bonding the really correct consideration? There have been a deal of quantum mechanical $a b$ initio calculations which proved: the charge redistribution in the valence electronic structure by forming Hydrogen-bonds, significantly differs from that induced by the electrostatic field of the surrounding molecules [6, 94, 96]. Also the experimental studies mentioned above confirmed that the pure electrostatic picture is not satisfactory for the representation of the H-bonding in water. However, the previous investigations on electronic structure of liquid water were not able to directly regard the electron density distributions of the H-bonded water molecules. The described observation of the spectral changes from gas phase to the condensed phases can only indicate the qualitative evolution of either a broad conduction band for condensed phases, or electronic structure variations of valence orbitals. No quantitive description of the electron density distributions in H-bonded water molecules could be concluded by those studies.

We also share the opinion that the nature of the H-bonding is much more complicated and further fascinating than just an electrostatic attraction. In the present work, we concern ourselves with the local orbital interaction picture of H-bonding in liquid water. However, we use a completely different and new experimental approach - an approach which particularly sheds light on the orbital-specific participation in H-bonding. We study the anisotropy parameter $\beta$ (or asymmetry 
parameter) in order to access orbital specific information in this regard. The $\beta$ parameter describes the angular distribution of photoelectron emission. Already in the early experiments on the angular distribution of photoelectrons ejected from molecules, Berkowitz and Ehrhardt suggested that the angular distribution may be useful as a source of information regarding the symmetry of the molecular orbital from which photoelectrons of a given energy are ejected [36]. The anisotropy parameter $\beta$ of a particular orbital reflects the electron density distribution of this orbital, or in other words the geometry of the orbital. Although the value of $\beta$ also depends on the energy of the ionizing photon, for a particular molecule, measurements with a fixed photon energy can provide valuable information. If we are able to determine the anisotropy parameters $\beta$ for the three outer valence orbital in liquid water and compare them with the corresponding ones in the gas phase, information can be obtained about how the orbitals are involved in the hydrogen-bond network. To be more precise, the change of the molecular electron densities or further characters of the molecular orbitals can be directly reflected by the variations of the anisotropy parameters for the valence orbitals. In addition, as an intermediate aggregate state between gas and liquid phase, small clusters are excellent model systems in this regard. Here, we trace changes in the shape of orbitals from gas over clusters to the bulk liquid. This allows new insights into $\mathrm{H}$-bonding in liquid water and may help to answer some of the fundamental questions in this regard: How to define an H-bond? What is the definition for such type of coordinate-covalent bonding as H-bonding? Can H-bonding be purely defined as the sharing of an electron pair of one molecule with an empty orbital of another? Which orbitals actually participate in H-bonding and to what extend? Here, we study the angular distribution of photoelectrons from water in the gas phase, in small clusters and in the liquid with the goal to understand the nature of the presumably most important intermolecular interaction: hydrogen bonds in liquid water. 


\subsection{Angle-dependent photoelectron spectra}

\subsubsection{PE spectra from liquid water}

In Fig 5.2 an exemplary photoelectron spectrum from a liquid water micro-jet (simplified as jet spectrum) is represented, which is measured at $p$-polarization of the ionizing radiation (see 4.2). Gas-phase contributions arise from the continuous evaporating of the liquid surface, and are indicated by the subscript $g$ (e.g., $1 b_{1, g}$ ). The sharp feature from the $1 b_{1, g}$ orbital with its well-known binding energy of $12.61 \mathrm{eV}$ provides a reference for the calibration function of the TOF-spectrometer.

A photoelectron spectrum of pure liquid water (indicated as liquid spectrum) can be obtained by subtracting the gas-phase contributions. For this, the pure

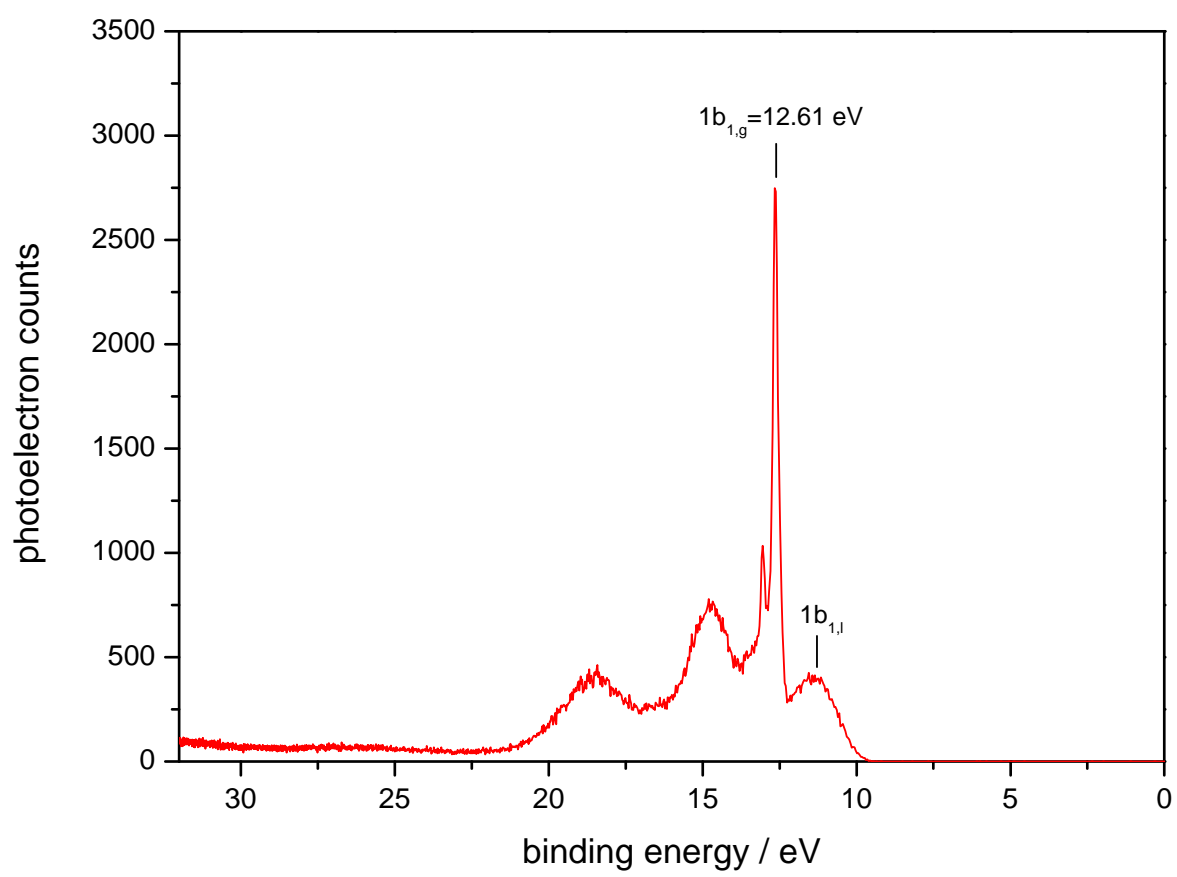

Figure 5.2: A photoelectron spectrum measured on water micro-jet (16 $\mu$ nozzle) with $p$-polarized EUV light $\left(\theta=0^{\circ}\right)$. The sharp feature from the $1 b_{1, g}$ orbital $(12.61 \mathrm{eV})$ provides a reference for the calibration function of the TOF-spectrometer. 
gas-phase spectrum (gas spectrum) is measured in an approach which is demonstrated in the right sketch b) in Fig.5.3. In part a), the experimental geometry for measuring spectra from the liquid micro-jet is shown. In contrast, with the geometry shown in b) only the photoelectrons ejected from isolated water molecules can fly through the skimmer and finally be detected. The gas-phase PE spectrum must be properly scaled in order to be comparable to the gas-phase contributions in the jet spectrum.

The above mentioned procedure is based on the approximation that the water molecules in the gas phase close above the surface of the liquid jet as well as those further away are randomly orientated and do not sense any potential of the latter. This approximation has only limited validity, which can be evidenced by comparing the $1 b_{1, g}$ peaks $(12.61 \mathrm{eV})$ of jet spectra with those of gas spectra: the $1 b_{1, g}$ peaks in jet spectra are somewhat broader than those in gas spectra. However, we expect that such an approximation does not influence the determination of $\beta$ and $\sigma$ very much and hence can be used for the purpose of this experiment (see details in section 5.3). Fig. 5.4 shows liquid spectra measured at different light polarizations.

Binding energy shift according to phase difference The most noticeable difference between the liquid and the gas-phase spectra is a binding-energy shift of

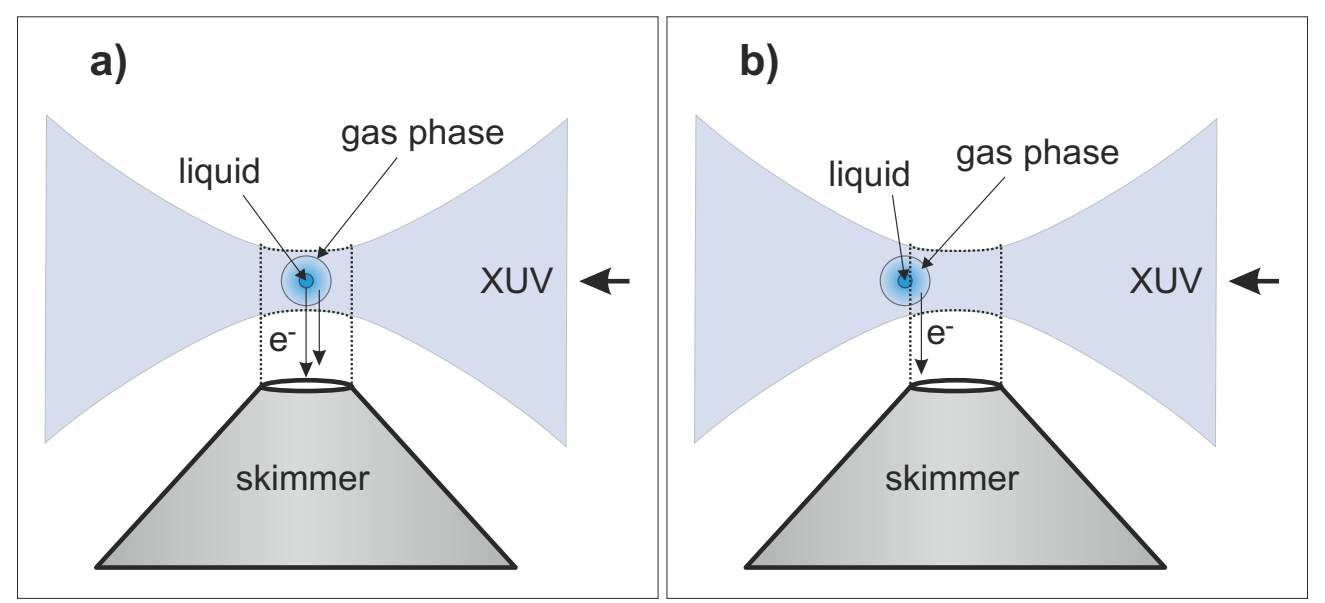

Figure 5.3: Sketch of the different approaches for measuring liquid jet and gas spectra, which are illustrated in a) and in b), respectively. 

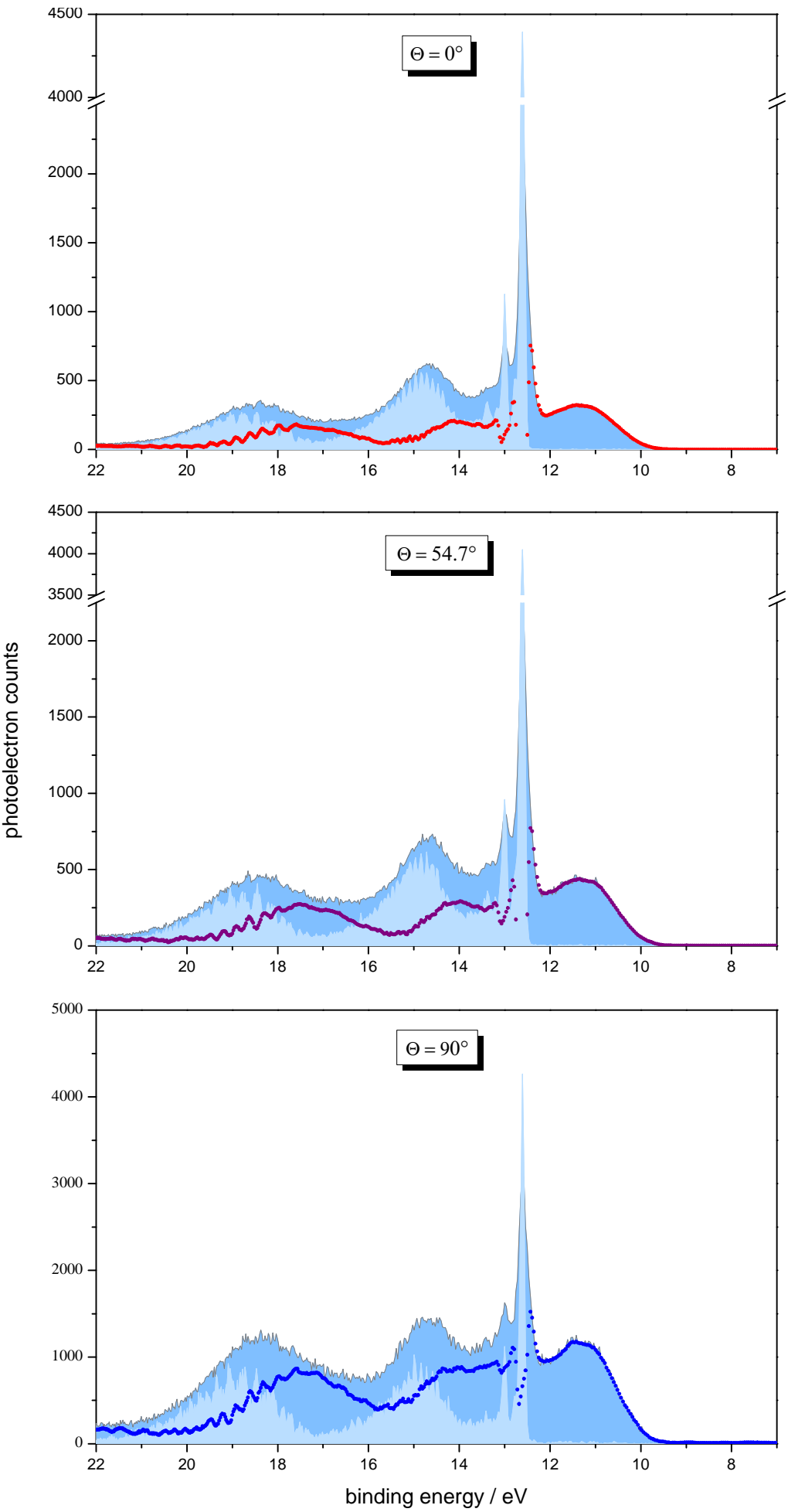

Figure 5.4: Polarization-dependent PE spectra from a liquid water micro-jet. Pure liquid spectra (colored scatter plot) can be obtained by subtracting the gas-phase contrigytions (light blue area) from the jet spectra (blue area). 
all water orbitals to lower values. The accurate shift of each feature related to a certain valence orbital will not be presented in this work. We refer to previous works [11, 68].

Winter et al. have observed similar shifts (around $1.4 \mathrm{eV}$ ) for the valence orbitals and pointed out that those are the net result of at least three different contributions: electronic polarization, surface dipoles, and changes in the orbitals due to H-bonding in the water network [11]. Winter et al. visualized the electronic polarization as follows: the outgoing photoelectrons sense the fast (fs time scale) polarization screening of the (liquid) environment around the molecular core. In this case, a final state effect is considered which lowers the energy of the resulting cationic state, compared to this state in the gas-phase. The photoelectron emission process is fast enough that any reorientation of the solvent water molecules can be neglected, and the binding energy of the electrons is directly reflected in their kinetic energy immediately after the emission. The observed shift in the order of $1-2 \mathrm{eV}$ is common in the photoemission of condensed (molecular) systems. Winter et al. applied the Born equation which describes the Gibbs free energy of solvation,

$$
\Delta G^{\text {Born }}=-\frac{z^{2} e^{2}}{8 \pi \epsilon_{0} R}\left(1-\frac{1}{\epsilon}\right)=E_{\mathrm{g}}-E_{\mathrm{aq}}
$$

to estimate the magnitude of the "polarization screening". Here, $E_{\mathrm{aq}}$ and $E_{\mathrm{g}}$ denote the respective binding energies in the solution and gas-phase. Applying $\epsilon=\epsilon_{\text {opt }} \simeq$ 1.8 and $R_{\mathrm{eff}}=2.24 \AA$, a value of $\Delta G^{\mathrm{Born}}=-1.4 \mathrm{eV}$ was obtained. Although this value was in good agreement with the shifts observed in their experiment, they admitted that this agreement was probably only a coincidence. The other cause for the binding energy shifts was - in their view - the oriented surface molecule dipoles. The magnitude of such a surface potential was equated with that of the permanent dipole of water molecule, which amounts to some ten $\mathrm{mV}$. The weight of this contribution was small according to their estimation. The only orbitalspecific contribution to the binding-energy shifts was identified by Winter et al. as the orbital changes resulting from H-bonding. However, it is particularly difficult to unravel this contribution from the others.

In our opinion, the truly interesting and extraordinary contribution to the binding-energy shift results from H-bonding. The simplified continuum model 
by Born neglects individual interactions between different molecules and can not reproduce any details of local coordination or further electronic properties of $\mathrm{H}$ bonded molecules in bulk water. Hence, the Born's solvation model is not really applicable for the accurate determination of the binding-energy shifts of water. Moreover, the effect of the surface potential on the liquid micro-jet can most likely be compensated due to the cylindrical surface. In addition, the binding energy shifts caused by the H-bonding must be determined with another approach. However, it is not the purpose of this work to find a description model to determine the gas-to-liquid binding-energy shifts for valence orbitals of water.

Peak broadening The spectral features of the $1 b_{1}, 3 a_{1}$ and $1 b_{2}$ orbitals in the liquid phase are apparently broadened compared to those in the gas phase. However, the amount of broadening is not the same for each orbital, which indicates that the contributions to the peak broadening are most probably specific for each of the three valence orbitals.

Nevertheless, there are similar factors doubtless which cause the main amount of broadening for all of them [11. Supposedly, the major broadening effect is associated with the broad distribution of various local environments around an individual water molecule in the liquid network. This is due to the fact that considered individually - each molecule in liquid water is surrounded by many other molecules positioned differently. These neighboring molecules perhaps are forming $\mathrm{H}$-bonds with the regarded molecule, breaking their $\mathrm{H}$-bonds or are just in the proximity, respectively. Also the outer surrounding molecules have interactions with the central molecule. In addition, the difference between the surface and the bulk network structure is considerable, since water molecules on the surface have fewer H-bonds on average.

The actual reason for the different amounts of broadening for the three orbitals is expected to be related to H-bonding which can also be regarded as intermolecular electronic interactions. By computing an ab initio MD trajectory, Hunt et al. tried to find out, to what extent the broadening of peaks in the density of states of liquid water is attributed to disorder in the molecular energy levels, and how much is due to band dispersion by electronic interactions between molecules [86]. They revealed that the statistical broadening is about $0.45 \mathrm{eV}$ for all bands independent 
of the character or position of the band. In contrast, the electronic broadening is rather sensitive to the nature of the orbital and was calculated to be $1.11 \mathrm{eV}$ for $1 b_{1}, 1.57 \mathrm{eV}$ for $3 a_{1}$ and $1.41 \mathrm{eV}$ for $1 b_{2}$. This indicated the dominance of electronic broadening as a result of $\mathrm{H}$-bonding. These calculated values are in no exact agreement with the observed peak widths from our study and previous studies by Winter et al. [11, but are of the same order of magnitude. Accordingly, we expect that the changes on the electronic structure of water molecules resulting from $\mathrm{H}$ bonding is of great relevance to the peak broadening of PE spectra due to the phase transition of water. Especially, the broad band in the range related to the $3 a_{1}$ orbital must be separately treated as follows, because of its unusual feature compared to the remaining orbitals.

Width of the $3 a_{1, l}$ feature An extending feature within the $3 a_{1, l}$-range can be observed in the liquid spectra measured at various light polarizations. The broadened structure of $3 a_{1, l}$ was observed in several studies using different approaches [11, 12, 17]. However, it is still unclear if this spectral structure is associated with one very broad but entire feature or a two-component feature. In [1] the latter is assumed and the cause for it is the Davydov-interaction which occurs between two adjacent identical molecules with different orientation in the unit cell. In contrast, in [12] no splitting was observed in the valence PE spectra of liquid water using $530 \mathrm{eV}$ photon energy. The authors supposed a strong $3 a_{1}-3 a_{1}$ orbital interaction within the formation of hydrogen-bond network. Each individual local geometry in the H-bonded network results in an unique orbital overlap. The sum of those local geometries is expected to result in an extraordinary smearing of the $3 a_{1}$ feature.

Because of the limitation of the used subtraction method for determining the pure water spectra, we can not yet conclude the above mentioned disputation. However, we tend to prefer the local orbital interaction picture of hydrogen bonding in liquid water to interpret the electronic nature of the $3 a_{1, l}$. This assumption is based on the results from the determination of the anisotropy parameter $\beta$ for the $3 a_{1}$ (see details in section 5.4. 
Angular distribution of Photoionization The liquid spectra for different angles $\theta$ are shown combined in Fig. 5.5. The spectra are scaled on the $1 b_{1, l}$-intensity, to demonstrate the differences between them. Comparing with the gas phase spectra (in the left upper corner), the $1 b_{1, l}$ and $1 b_{1, g}, 3 a_{1, l}$ and $3 a_{1, g}$ features look quite similar, while $1 b_{2, l}$ and $1 b_{2, g}$ are distinctly different - the $1 b_{2, l}$-intensity changes at the three angles are less pronounced than those of $1 b_{2, g}$. Notice, that this does not indicate that the $1 b_{2}$ changes the most when water molecules are brought together in the liquid phase. The situation may just reverse if the spectra scaling is related to $1 b_{2, l}$. A proper interpretation for the varying angular distribution of photoemission from water molecules in different phases can only be made after the determination of anisotropy parameters $\beta$.

In particular, the angular distribution features of $1 b_{1}$ orbital will be regarded separately. For it, we rescale the three spectra, means to the similar $1 b_{1, g}$-intensities in the corresponding gas spectra (see Fig.5.6). Contrasting with the gas phase spectra, the distinctions of the $1 b_{1, l}$ at the three angles $\theta=0^{\circ}, \theta=54.7^{\circ}$ and $\theta=90^{\circ}$ are more considerable. Due to the similar argument mentioned above, the conclusion about this observation will be presented in section 5.4 .

\subsubsection{PE spectra from small water clusters}

Small water clusters exhibit comparable spectral features to liquid water, which is presented in the following. Because of the statistic thermodynamic distribution of the cluster size, one gets water monomers in the majority, even if it is purposed to obtain small clusters via supersonic expansion. Therefor, all the cluster measurements includes mainly monomer intensity and the contribution from the small clusters is so small that a similar subtraction method, as for liquid water spectra, is not really satisfactory. On this account, we do not intend to determine pure cluster spectra, but concern ourselves only with the property of $1 b_{1}$ orbital in the small water aggregates (indicated as $1 b_{1, c l}$ ), since the photoelectron feature originated from $1 b_{1, c l}$ is nearly isolated from that of $1 b_{1, g}$ (see Fig.5.7).

In Fig. 5.7, three PE spectra of water are presented, which are measured from the liquid jet, small clusters and gas phase, respectively. Viewing the three spectra, one notes the apparent difference between them. In particular, in the range 


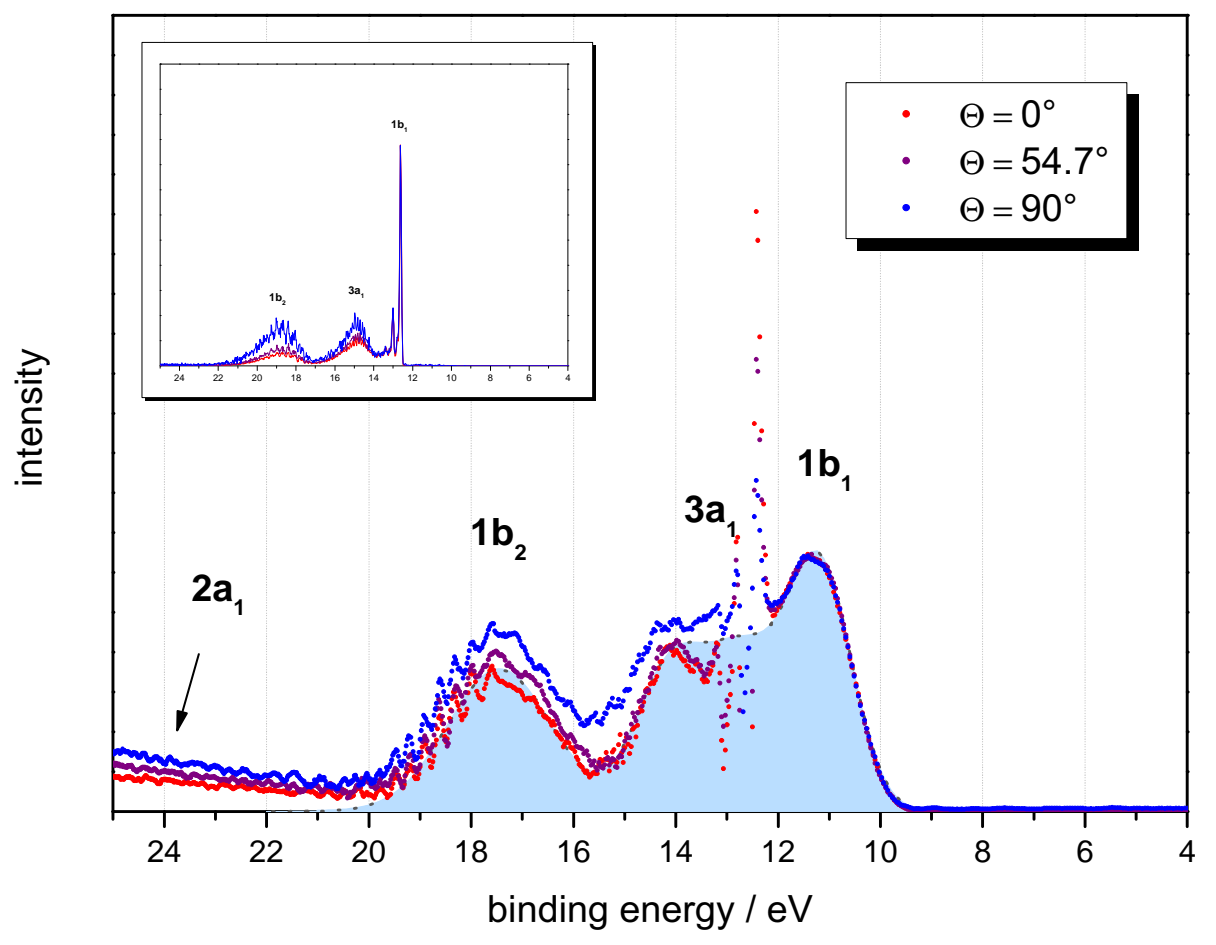

Figure 5.5: Combined liquid PE spectra at different angles $\theta$. The photoelectron counts of those spectra are scaled so that the intensities of $1 b_{1, l}$-peaks are similar. The remaining weak structure in the $1 b_{2}$ range results from imperfections in the scaling of the subtracted monomer spectrum. Since the feature of the spectra are not directly measured, but scaled, we use here intensity as vertical label, in spit of photoelectron counts. For comparison, gas phase spectra are shown in the left corner.

of $1 b_{1}$ orbital there are apparent binding energy shifts from gas phase $(12.60 \mathrm{eV})$, via cluster $(12.06 \mathrm{eV})$ to the liquid phase $(11.20 \mathrm{eV})$. The peak width of the $1 b_{1, c l}$ $(F W H M=1.47 \mathrm{eV})$ and $1 b_{1, l}(\mathrm{FWHM}=1.50 \mathrm{eV})$ are not very different because the accounts for the broadening related to $1 b_{1, g}$ are similar assumedly (see subsection 5.2.1). 


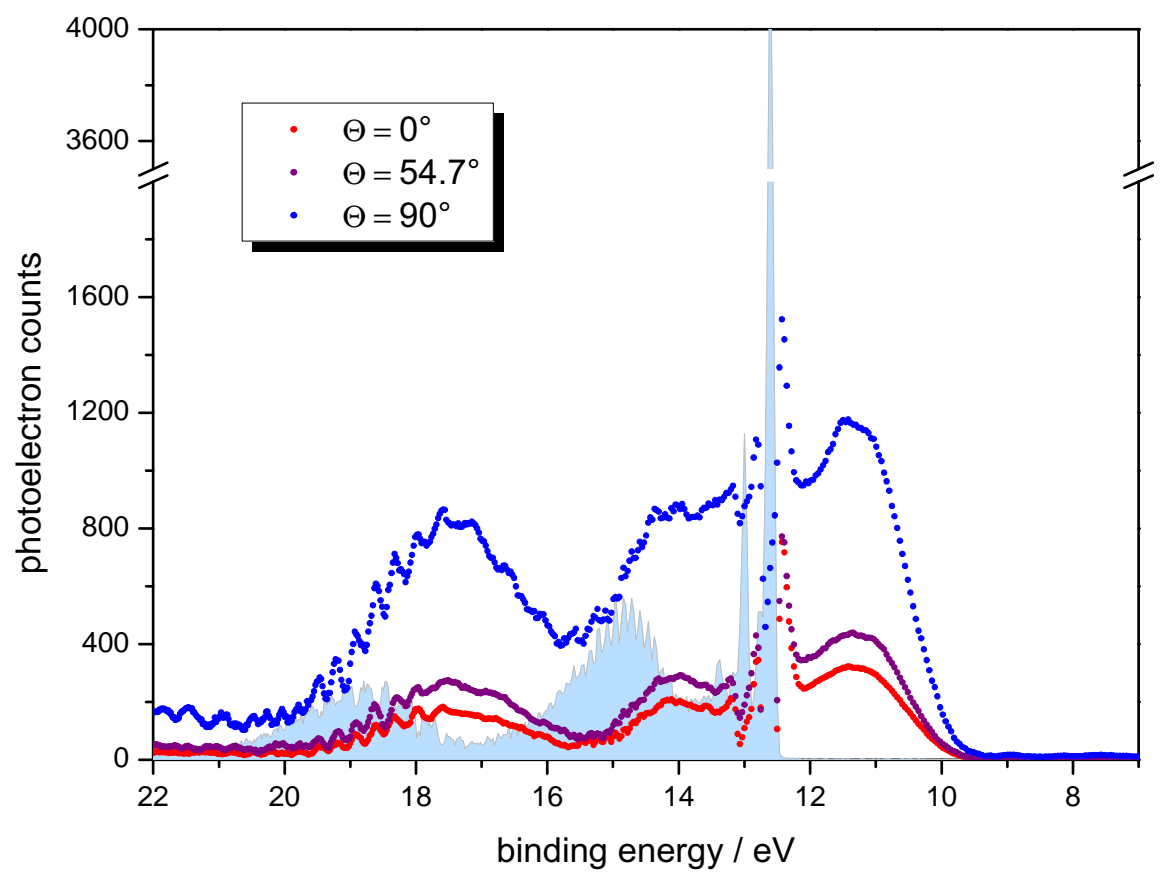

Figure 5.6: Rescaled liquid spectra at different angles $\theta$. The photoelectron counts of those spectra are scaled so that the $1 b_{1, g}$-peaks in the corresponding gas spectra are the same. Within the light blue area, a gas spectrum measured with $p$-polarized EUV light is shown in order to demonstrate the scaling operation. Compare this figure also with Fig. 5.4 .

Mean cluster size The mean size of the water clusters examined in this work can be estimated with two different approaches. Firstly, we apply the formula deriving from the scaling laws method to determine $\langle n\rangle$ (see Eq.4.3). The optimized values of the required empirical parameters for water were found in reference 78 to be $a=1.886, q=0.634$ and $D=11.6$. In the measurements of water clusters in the present work, the reservoir had a temperature $T_{r}=60^{\circ} \mathrm{C}$ and the nozzle $T_{n}=75^{\circ} \mathrm{C}$ which correlates with $p_{0}=199$ mbar. As a result, the mean cluster size should be $\langle n\rangle=13$. 


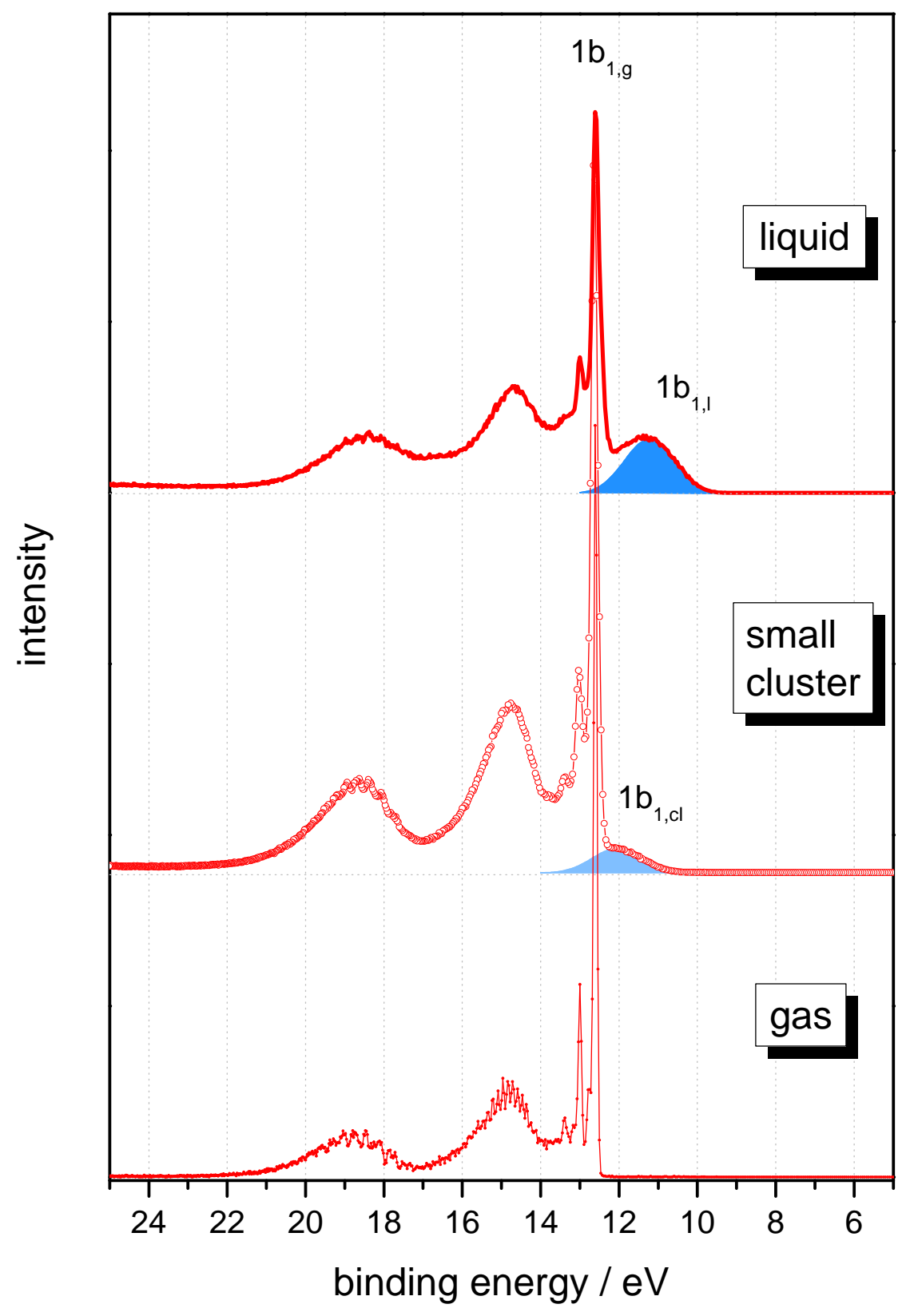

Figure 5.7: Photoelectron spectra of water molecules, small water clusters and liquid water measured with $p$-polarized EUV light. Notice the binding energy shifts from gas phase, via cluster to liquid phase, in particular the shift of the $1 b_{1}$ band. 
There is also another way to estimate the mean size of the clusters in photoelectron spectroscopy, which is provided in [13. In that work, clusters of different sizes $(n=4.5,17,65$ and 108) were investigated using synchrotron radiation. The position of the $1 b_{1}$-peak maximum was found to depend on the cluster size and the respective values extrapolate linearly toward that of liquid water when plotted as a function of inverse cluster radius, which is proportional to $n^{-1 / 3}$. We adopt the results from them to estimate the mean cluster size probed in our experiment. The empirically established linear relationship between ionization energy and inverse cluster radius is

$$
n=\left(\frac{\Delta G}{\Delta G-E_{g}+E_{n}}\right)
$$

which can be applied to estimate the mean cluster size in an ionization experiment. Here, $E_{g}$ and $E_{n}$ denote the binding energy of the $1 b_{1, g}$-peak $(12.61 \mathrm{eV})$ and the maximum of the $1 b_{1, c l}$-peak, which originates from a water cluster distribution $\left(\mathrm{H}_{2} \mathrm{O}\right)_{n}$ with a mean cluster size $n . \Delta G$ is the Gibbs free energy of solvation, which is given in equation 5.1 as $1.4 \mathrm{eV}$. Based on Eq.5.2, the mean cluster size in our experiment is estimated to be 4 or 5 , whereby the observed $E_{n}=12.06 \mathrm{eV}$ is used. Due to the cubic dependence on the energy difference, the error of such a calculation is around $\pm 30 \%$.

The disagreement between the two different approaches to determine the mean cluster size is associated with the following facts [104]. In the experiment, on which Eq.4.3 is based, the vacuum conditions for the expansion were better due to a more powerful diffusion pump $(3000 \mathrm{l} / \mathrm{s})$, compared to the turbo-molecular pump used in our experiment $(1000 \mathrm{l} / \mathrm{s})$. Also, the fixing positions of the vacuum pump are different in both experiments: the pump is located directly near the nozzle in their equipment while this is not the case in our setup. This leads to varying pressure conditions of the expansion. In our experiment, there are more background water monomers in the cluster beam which may affect the temporal development of the cluster size. At a short distance from the nozzle opening it may thus be that the final cluster size is not yet developed and that the cluster size changes with varying probe position. In our experiment, the cluster volume $6 \mathrm{~mm}$ away from the nozzle opening is probed with EUV radiation, while the cluster size is firstly analyzed in a distance of $1.5 \mathrm{~m}$ from the nozzle hole in the setup of Bobbert et al. [78]. Summarizing, the empirical formula above may not be suited 
to determine the mean cluster size in our experiment. In addition, validity of this formula is limited for very small clusters $(n<10)$.

The sensibility of photoelectron spectra for binding energy shift with different aggregation degree of molecules is reliable, to our best consideration. Hence, for now we assume that the mean cluster size in our photoionization experiment is about 5 .

In Fig.5.8 different angular distribution properties are visible for $1 b_{1, g}$ and $1 b_{1, c l}$. The cluster spectra are scaled so that the $1 b_{1, g}$-peaks exhibit the same intensity for all of the observation angles. The $1 b_{1, c l}$-peak has the strongest intensity for $s$-polarized EUV light. The complete understanding of this experimental observation can be achieved only if the angular distribution of the photoionization of the $1 b_{1}$ orbital in small clusters is explicated by determining their $\beta$ value.

\subsection{Determination of the anisotropy parameter $\beta$}

As described in section 2.4, the general form for the differential photoionization cross section of a certain orbital $i$ is expressed as

$$
\frac{d \sigma_{i}}{d \Omega}(\theta)=\frac{\sigma_{i}}{4 \pi}\left[1+\frac{\beta_{i}}{4}\left(1+3 P_{1} \cos 2 \theta\right)\right] .
$$

Here $\sigma_{i}$ represents the total cross section which has a dimension of area per atom or molecule and is a function of the incident photon energy. The anisotropy parameter or asymmetry parameter $\beta$ can have values from -2 to 1 . $\theta$ is the angle between the direction of the ejected electron and the polarization vector of the incident light. $P_{1}$ is the Stokes parameter and denotes the degree of linear polarization. Eq.5.3 applies strictly to photoionization of atoms, but can also be used for other species, under the condition that they are randomly oriented. In the micro-jet experiment, this equation is approximately applicable [75]. The cylindric surface of the sample hinders the molecules to align themselves in the same orientation.

For linearly polarized light $P_{1}=1$ and equation 5.3 can be transformed to

$$
\frac{d \sigma_{i}}{d \Omega}(\theta)=\frac{\sigma_{i}}{4 \pi}\left[1+\frac{\beta_{i}}{4}(1+3 \cos 2 \theta)\right] .
$$




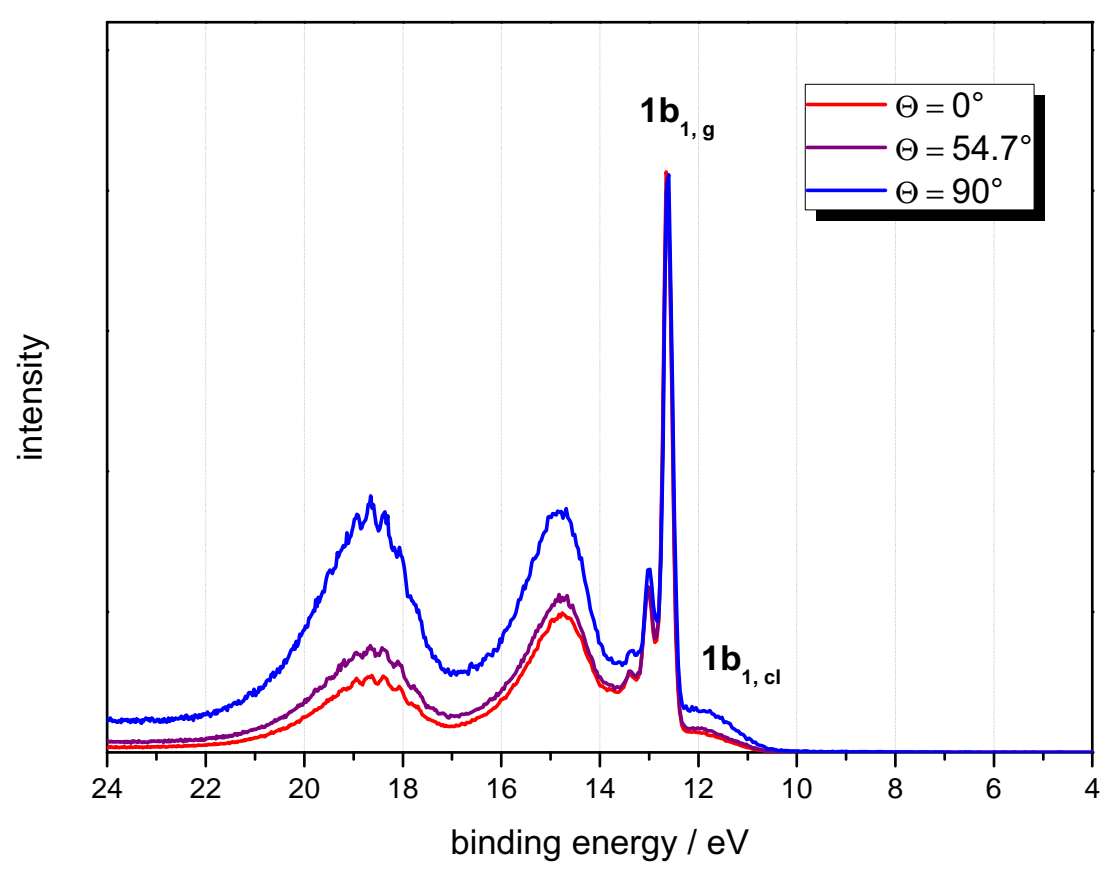

Figure 5.8: Photoelectron spectra of small water clusters measured with differently polarized EUV light. These spectra are associated with varying small-sized clusters $\left(\mathrm{H}_{2} \mathrm{O}\right)_{n}(n=1,2,3 \ldots 10)$. These spectra are scaled on the intensity of $1 b_{1, g}$-peak, in order to demonstrate the altered angular distribution going from gas phase to small clusters.

For three special angles $\theta$, the following equations can be determined:

$$
\begin{gathered}
\left(\frac{d \sigma_{i}}{d \Omega}\right)_{\|}=\frac{d \sigma_{i}}{d \Omega}\left(\theta=0^{\circ}\right)=\frac{\sigma_{i}}{4 \pi}\left(1+\beta_{i}\right) \\
\left(\frac{d \sigma_{i}}{d \Omega}\right)_{m}=\frac{d \sigma_{i}}{d \Omega}\left(\theta=54.7^{\circ}\right)=\frac{\sigma_{i}}{4 \pi} \\
\left(\frac{d \sigma_{i}}{d \Omega}\right)_{\perp}=\frac{d \sigma_{i}}{d \Omega}\left(\theta=90^{\circ}\right)=\frac{\sigma_{i}}{4 \pi}\left(1-\frac{\beta_{i}}{2}\right) .
\end{gathered}
$$

In order to obtain the relative cross sections of all the photoionization channels (orbitals) of a system, theoretically one only needs to measure the spectrum at the 
magic angle $\theta=54.7^{\circ}$. The photoionization cross section $\sigma_{j}$ of orbital $j$ related to $\sigma_{i}$ of orbital $i$ can be determined from the ratio of the corresponding peak areas $A$ :

$$
\frac{\sigma_{j}}{\sigma_{i}}=\frac{A_{j}}{A_{i}}
$$

However, $\sigma_{i}$ values obtained in this way are often not consistent with the true photoionization cross section, which is due to the following limitations of such experiments:

* It is difficult to determine and to separate the effects of auto-ionization.

* Inaccuracies, such as the inexact adjusting or determining of the angle $\theta$ can falsify the $\sigma_{i}$ values.

* The problem is further complicated when a monochromator is used to produce the ionizing flux, since radiation emerging from a monochromator is only partially polarized and the degree of polarization varies with wavelength.

* Additional effects can also be caused by imperfect analysis of the photoelectron spectra, in particular, when the ionization channels exhibit spectra features extending into each other. Often, this is the case, when polyatomic molecule systems in condensed phases are investigated.

For the experimental determination of $\beta_{i}$, in an ideal case, only two measurements at two angles $\theta$ (e.g. $\theta=0^{\circ}$ and $\theta=54.7^{\circ}$ ) are required. Therefor, two photoelectron analyzers should be fixed at different observation angles to detect the photoelectron intensities at the same time. In this case, the polarization vector of the ionizing radiation is constant. Then $\beta_{i}$ can be obtained from:

$$
\beta_{i}=1-\frac{A_{i, \|}}{A_{i, m}}
$$

In our experiment, this determination method for $\beta$ is not applicable, because we do not have two spectrometers to record photoelectron spectra at the same time. In spite of that, we tune the polarization vector of the incident radiation with a $\lambda / 2$ plate - at a fixed TOF photoelectron spectrometer geometry - to collect spectra at different $\theta$ (see subsection 4.2). This has the advantage that the ionized 
sample volume observed at a particular angle is always the same while this is not the case, if two spectrometers collect the ejected electrons at the same time.

Nevertheless, there are several facts limiting the prospects of our experiment as follows:

* A quantitative estimation of photon flux at a certain polarization of EUV light is not quantitatively available.

* There are probably fluctuations in the quality of the EUV light or the micro-jet flux, which may not be compensated by long measurement times.

* The target quantity is not exactly known, in particular, the ratio of probed water molecules in the liquid to those in the gas phase.

All of these facts make the determination of $\beta$ using conventional evaluation methods difficult. Also large errors can be expected. Therefore, we perform a new procedure to obtain the values of $\beta$, which do not require knowledge of photon flux or probed sample quantity in each measurement.

As an example to demonstrate how the $\beta$ 's are obtained in this experiment, two orbitals, 1 and 2 of the same molecule species will be considered. Since the photoelectrons ejected from these orbitals are always detected simultaneously, the eventual fluctuations of the photon flux or minimal changes of the radiation direction do not play a role any more.

At magic angle $\theta=54.7^{\circ}$,

$$
\frac{\sigma_{1}}{\sigma_{2}}=\left(\frac{I_{1}}{I_{2}}\right)_{m}
$$

is valid, where $I_{i}$ denotes the photoelectron counts emitted from a certain orbital $i$.

For $p$-polarization, the following relationship exists:

$$
\left(\frac{I_{1}}{I_{2}}\right)_{\|}=\frac{\frac{\sigma_{1}}{4 \pi}\left(1+\beta_{1}\right)}{\frac{\sigma_{2}}{4 \pi}\left(1+\beta_{2}\right)}=\frac{\sigma_{1}}{\sigma_{2}} \cdot \underbrace{\frac{1+\beta_{1}}{1+\beta_{2}}}_{a}
$$

where $a=\left(1+\beta_{1}\right) /\left(1+\beta_{2}\right)$. Putting 5.10 in 5.11, one gets

$$
a=\frac{\left(I_{1} / I_{2}\right)_{\|}}{\left(I_{1} / I_{2}\right)_{m}}=\frac{I_{1, \|} / I_{1, m}}{I_{2, \|} / I_{2, m}}=\frac{\left(P_{\|} / P_{m}\right)_{1}}{\left(P_{\|} / P_{m}\right)_{2}} .
$$

where $P_{i}$ denotes the peak height of the orbital $i$ in a spectrum measured at a certain EUV polarization. The third part of Eq.5.12 is based on the assumption that 
the peak width belonging to one orbital does not change by tuning the polarization direction of the EUV radiation, as it is observed in our experiments.

For $s$-polarized light, analog to Eq.5.11 and Eq.5.12, the following formulae are given:

$$
\begin{gathered}
\left(\frac{I_{1}}{I_{2}}\right)_{\perp}=\frac{\sigma_{1}}{\sigma_{2}} \cdot \underbrace{\frac{2-\beta_{1}}{2-\beta_{2}}}_{b} \\
b=\frac{\left(I_{1} / I_{2}\right)_{\perp}}{\left(I_{1} / I_{2}\right)_{m}}=\frac{I_{1, \perp} / I_{1, m}}{I_{2, \perp} / I_{2, m}}=\frac{\left(P_{\perp} / P_{m}\right)_{1}}{\left(P_{\perp} / P_{m}\right)_{2}}
\end{gathered}
$$

If $a$ and $b$ have been computed, we can solve the following equation system with two unknowns $\beta_{1}$ and $\beta_{2}$ :

$$
\frac{1+\beta_{1}}{1+\beta_{2}}=a
$$

and

$$
\frac{2-\beta_{1}}{2-\beta_{2}}=b
$$

$\beta_{1}$ and $\beta_{2}$ are given as:

$$
\beta_{1}=\frac{2 a+b-3 a b}{a-b}
$$

and

$$
\beta_{2}=\frac{3-a-2 b}{a-b}
$$

The advantages of the procedure described above are:

* The phase state of the system under investigation does not matter. This method can be applied to liquid or gas phase.

* Photon flux or the probed sample volume do not have to be known.

* To determine the angle distribution of photoelectron emission from a certain ionization channel in a system, no scaling of the spectra is needed. This is important, when the spectra are not measured simultaneously or with the same recording time.

There are only a few requirements for this procedure: 
* The spectral ranges of the considered channels must be somehow isolated from each other and

* their angular photoemission distributions must be non-identical.

The latter requirement is limited mathematically, because if the values of $\beta_{1}$ and $\beta_{2}$ are identical, $a$ and $b$ will be equal and furthermore, Eq.5.17 and Eq.5.18 will be undefined. This also means that the more different the angular distributions are, the smaller is the calculated error. Due to these requirements, $\beta$ values for $1 b_{1}, 3 a_{1}$ and $1 b_{2}$ orbitals are determined for water, both in the gas and liquid phase while only the $\beta$ value for $1 b_{1}$ can be obtained for small water clusters in this way.

\subsection{Results and discussion}

In this section we will present at first the different angular distributions of photoelectron emission for water in different phases - gas, small clusters and liquid. These have been computed by carrying out the procedure explained in section 5.3 and are tabulated in Tab.5.1. It is important to note that the $\beta$ values in this work are obtained for a photon energy of $38.7 \mathrm{eV}$. The dependence of $\beta$ on the ionization energy has been investigated by Banna et al. [39] and will not be discussed here in detail. After the presentation of the asymmetry parameter $\beta$ for different orbitals, we comprehensively discuss the electronic structure changes arising from hydrogen bonding among water molecules.

In order to obtain the asymmetry parameter $\beta_{i}$ for liquid water, we first determine the fractions $a$ and $b$. They are defined in the previous section 5.3. Only the peak heights attributed to each orbital are required. We do not need to fit peaks in the spectra carefully, to assign them to particular orbitals. This will cause imprecisions and hence it is misadvised because of the aforementioned reasons. Even any scaling operation is not essential to compute $a$ and $b$ using this method. Notably, different combinations of reference orbitals (as orbital 1 and 2) can be performed to get the $\beta$ values for each orbital. For instance, $\beta$ for $1 b_{1}$ (orbital 1 ) can be determined by taking $3 a_{1}$ or $1 b_{2}$ as a partner (orbital 2). In this way, the values of $\beta$ obtained from different approaches, can be compared with each other and thus rechecked. 


\begin{tabular}{|c|c|c|c|c|c|}
\hline \multirow{2}{*}{$\begin{array}{l}h \nu=38.7 \mathrm{eV} \\
\text { phase }\end{array}$} & \multirow[b]{2}{*}{ orbital } & \multirow[b]{2}{*}{$\beta_{i}$} & \multirow[b]{2}{*}{ rel. $\sigma_{i}$} & \multicolumn{2}{|c|}{ Banna et al. 39. } \\
\hline & & & & $\beta_{i}$ & rel. $\sigma_{i}$ \\
\hline \multirow[t]{3}{*}{ gas } & $1 b_{1, g}$ & 1.4 & 1 & 1.38 & 1 \\
\hline & $3 a_{1, g}$ & 1.1 & 0.88 & 1.12 & 0.88 \\
\hline & $1 b_{2, g}$ & 0.7 & 0.94 & 0.71 & 0.94 \\
\hline \multirow[t]{3}{*}{ small cluster } & $1 b_{1, c l}$ & 1.0 & & & \\
\hline & $3 a_{1, c l}$ & - & & & \\
\hline & $1 b_{2, c l}$ & - & & & \\
\hline \multirow[t]{3}{*}{ liquid } & $1 b_{1, l}$ & 0.8 & 1 & & \\
\hline & $3 a_{1, l}$ & 0.7 & 0.88 & & \\
\hline & $1 b_{2, l}$ & 0.6 & 0.94 & & \\
\hline
\end{tabular}

Table 5.1: Experimental results for the PE angular distribution $(h \nu=38.7 \mathrm{eV})$ of gas, small clusters and liquid water. The error of the $\beta_{i}$ values amounts to $10 \%$. The reference values originate from the synchrotron PES studies performed at the BESSY (Berlin Germany) by Banna et al. in 1986. The $\sigma_{i}$ values used in our studies are all adopted from their results. Note details in text of this section.

To test the self-consistency of the presented calculation procedure, we also compute $\beta_{i}$ for the three outmost water valence orbitals $1 b_{1}, 3 a_{1}$ and $1 b_{2}$ in the gas phase. The resulting values, presented in Tab.5.1, are in very good agreement with those reported by Banna et al. in [39]. However, we have some problems to reproduce their values for the relative photoionization cross section $\sigma_{i}$, because of several non-elusive inaccuracies in our experiments: the toroidal grating used here for selecting the $25 \mathrm{th}$ harmonic of the $800 \mathrm{~nm}$ fundamental as ionizing photon energy has an somehow asymmetric profile if the requirement for point-to-point imaging is not guaranteed. The sensitivity of the TOF photoelectron spectrometer is not calibrated for detecting photoelectrons with different kinetic energy. With our experimental setup, it is difficult to determine an accurate detection efficiency function for our photoelectron spectrometer. But carrying out calibration mea- 
surements at a synchrotron beamline could allow to determine properties of our TOF spectrometer. Nevertheless, those supposed interference factors do not play an essential role in our determination procedure of $\beta_{i}$. This is confirmed by the good agreement of our results with those from Banna et al., which are the most acknowledged and cited values. In addition, we adopt the relative $\sigma_{i}$ values of $1 b_{1}$, $3 a_{1}$ and $1 b_{2}$ from [39] to portray a complete picture of the photoelectron emission from the three outmost valence orbitals of free water molecules, which is presented in Fig. 5.9 .

Due to the limitations of our cluster generation method (see 4.5 and 5.2 .2 ), we are not able to identify the asymmetry parameter $\beta_{i}$ of every valence orbital for small water clusters. Only $\beta$ for the $1 b_{1, c l}$ orbital is determined. By comparison of this value with the $\beta$ values of the $1 b_{1, g}$ and the $1 b_{1, l}$ signals, valuable insights into the electronic structure rearrangements of water molecules from gas over small aggregates to the condensed state can be obtained.

In order to simulate the total angular distribution of photoelectron emission for the three ionization channels $1 b_{1}, 3 a_{1}$ and $1 b_{2}$, we need at least the photoionization cross section ratios between them, if the total cross sections are not available. Unfortunately, there have not been so far any known studies with this purpose. However, we assume that the partial photoionization cross sections $\sigma_{i}$ of orbitals are only slightly influenced by the intermolecular interactions, due to the nature of the photon absorption in the ionization process. In other words, electronic density changes in the ionic final states, stabilized by interactions between neighboring molecules, does not strongly affect the ionization cross section. Even if there are any effects associated with the phase differences, those are expected to be quite similar for all the valence orbitals. In contrast, $\beta$ is more sensitive to phase changes, which will be discussed later in detail. Comparable observations and interpretations have been made in [105]. Because of the reasons above, we use the $\sigma_{i}$ values from the PE studies on gaseous water (see Tab.5.1) and present the angular distribution of the three orbitals in space, utilizing the $\beta_{i}$ values determined in this work (Fig.5.10).

Considering the two illustrations in Fig. 5.9 and 5.10, one can immediately notice the differences between them - the distribution is more isotropic in the liquid case 


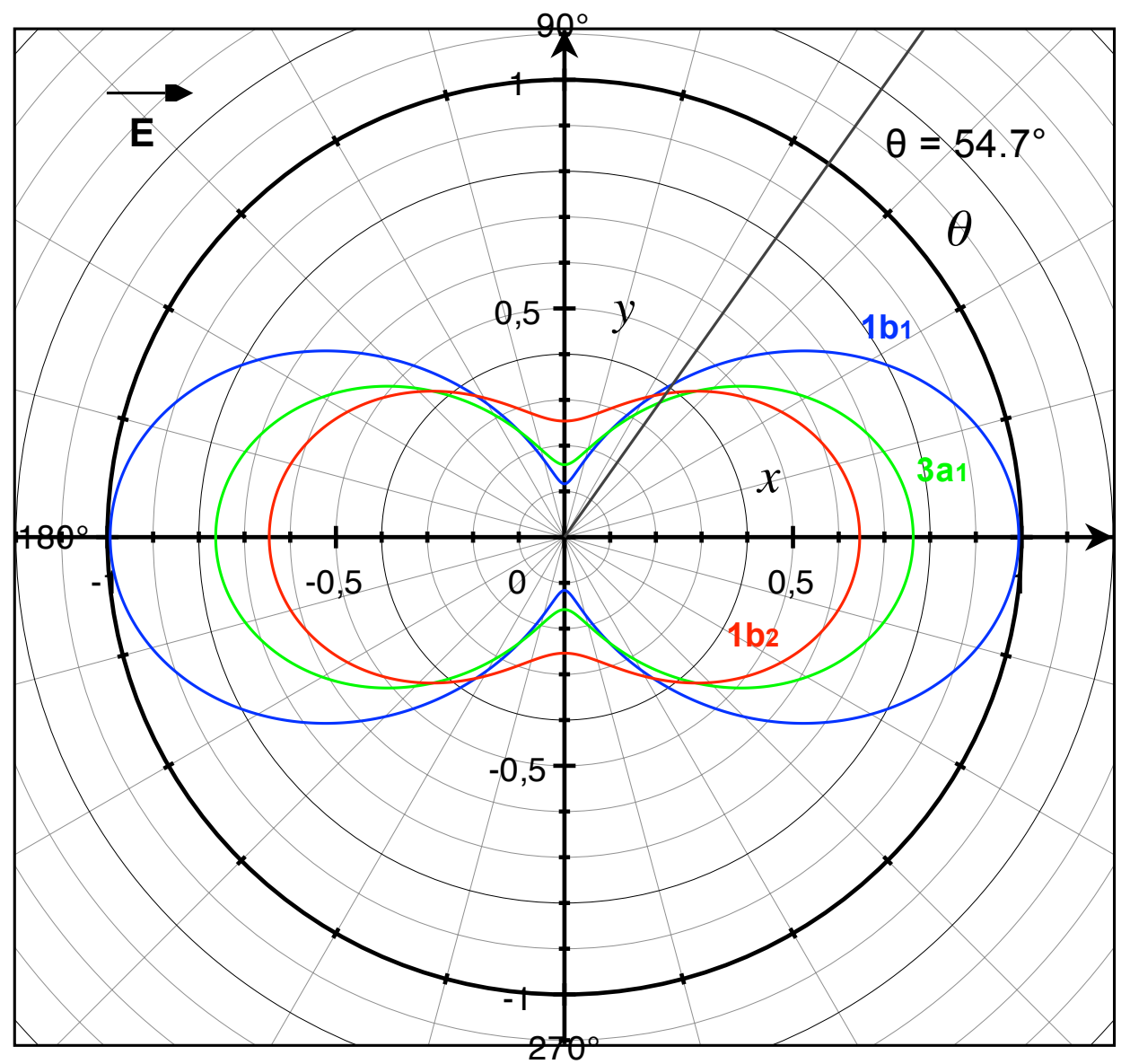

Figure 5.9: Angular distribution of photoionization for $1 b_{1}$ (blue), $3 a_{1}$ (green) and $1 b_{2}$ (red) orbitals of gaseous water. The ionizing radiation has a photon energy of $38.7 \mathrm{eV}$ and propagates perpendicularly to the xy-plane through the origin. Polarization vector of the EUV light and the magic angle are indicated, respectively. These curves are based on our experimentally obtained $\beta_{i, g}$ values and the $\sigma_{i, g}$ given by [39] in Tab.5.1. The absolute values of the $\mathrm{x}$ - and $\mathrm{y}$-axis have no relevance. 


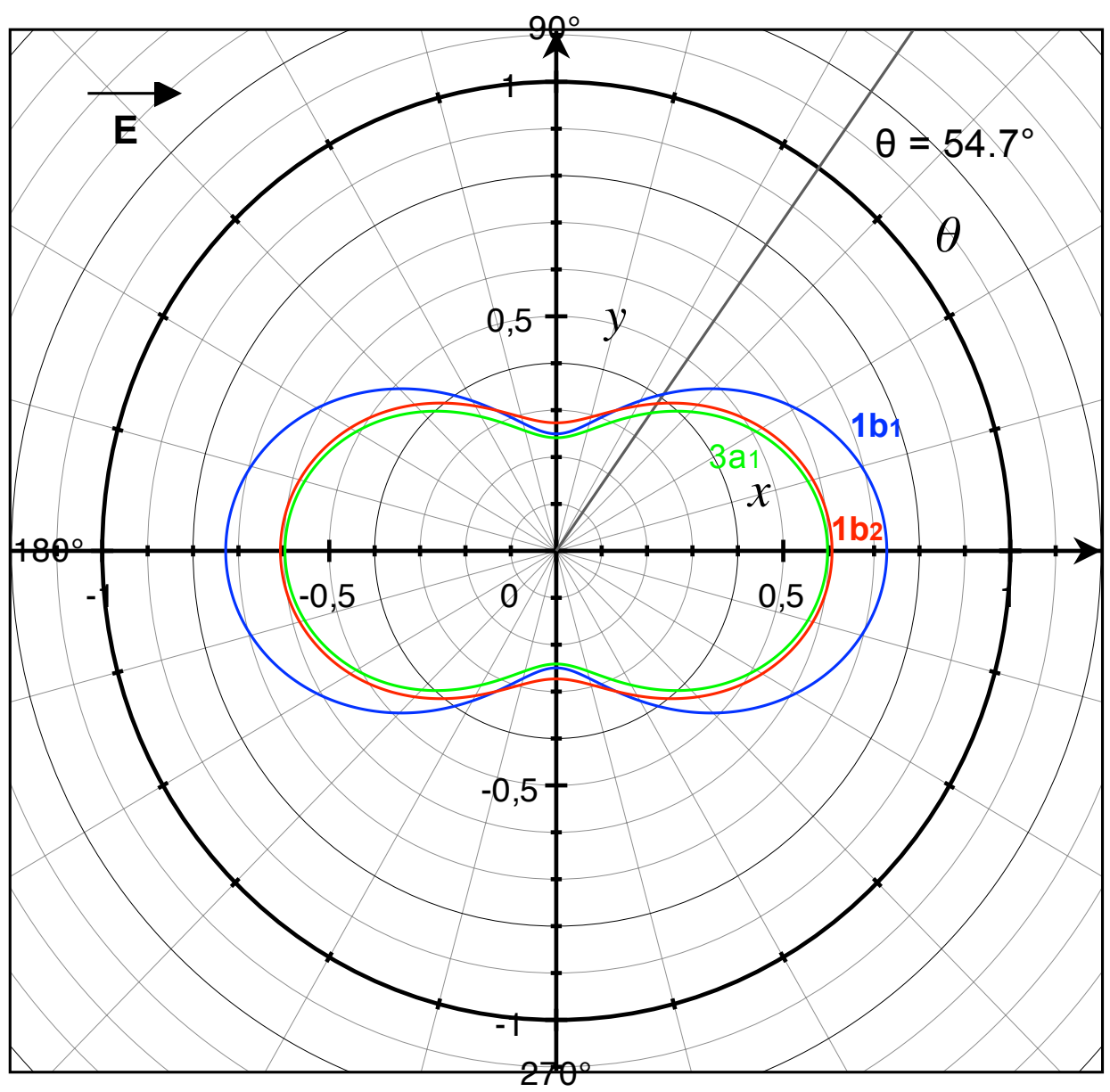

Figure 5.10: Angular distribution of photoelectrons from the $1 b_{1}$ (blue), $3 a_{1}$ (green) and $1 b_{2}$ (red) orbitals of liquid water. The ionizing photon energy is $38.7 \mathrm{eV}$. These curves are based on our experimentally obtained $\beta_{i, l}$ values and $\sigma_{i, g}$ in Tab.5.1, assuming that the $\sigma_{i}$ do not change for water molecules in different aggregate states. Since the same $\sigma_{i}$ are applied to create Fig. 5.9 and this one, a direct comparison of their magnitudes is possible. Note that $3 a_{1}$ shows less photoelectron emission at any direction than $1 b_{2}$. This is in contrast to Fig. 5.9 . 
for all of the orbitals. Nevertheless, there are also individual changes, which makes it necessary to regard each orbital separately.

At first, we look at the case of $1 b_{1}$ by depicting its angular distributions of photoelectron emission for different phases in Fig.5.11. Here, we take the anisotropy parameter not only for $\beta$ of $1 b_{1, g}$ and $1 b_{1, l}$ into account but also the $1 b_{1, c l}$ in small water clusters. The same value of $\sigma$ was applied for all the three aggregate states. As can be seen, the photoionization distribution of this orbital becomes more and more isotropic when going from isolated molecules in the gas phase over clusters to the bulk liquid, which implies increasing electronic interactions between the molecules. In particular, the forming of hydrogen-bond networks is the major cause of this effect. The most interesting observation is the significant change of the angular distribution in the transition from the gas phase to small clusters, even when the mean size of the clusters is only around $n=5$. The interpretation of this phenomenon will be discussed in the following.

In analogy to the way described above for the $1 b_{1}$ orbital, we determine the asymmetrical photoemission distribution of the $3 a_{1, g}$ and $3 a_{1, l}$ signals in Fig. 5.12 and the $1 b_{2, g}$ and $1 b_{2, l}$ signals in Fig.5.13. A similar change of the photoelectron emission anisotropy is also observed in the case of the $3 a_{1}$ orbital when the water molecules are involved in H-bond networks. The situation is considerable different for the $1 b_{2}$ orbital. The angular distribution for the $1 b_{2}$ orbital in the gas phase is apparently more isotropic than that of the $1 b_{1}$ or $3 a_{1}$. In the liquid, the distribution anisotropy for $1 b_{2}$ becomes only slightly more isotropic. This dissimilarity reflects the different participation of the $1 b_{2}$ orbital in the H-bond network compared to both $1 b_{1}$ and $3 a_{1}$.

Due to the mathematic definition of $\beta$ and the $\cos ^{2} \theta$-shaped distribution, it is not meaningful to determine the percentage variation of the value of $\beta$, and use it to figure out if a particular orbital is more or less involved in hydrogen bonding than an other one. Hence, we do not intend to perform such a comparison but consider merely the qualitative influence of the varying $\beta$ parameter on the shape of the angular photoelectron distribution. From our results discussed above, it is obvious that the degree of photoionization anisotropy of all the three valence orbitals is lowered in the liquid phase, however for the $1 b_{1}$ and $3 a_{1}$ orbitals, the 


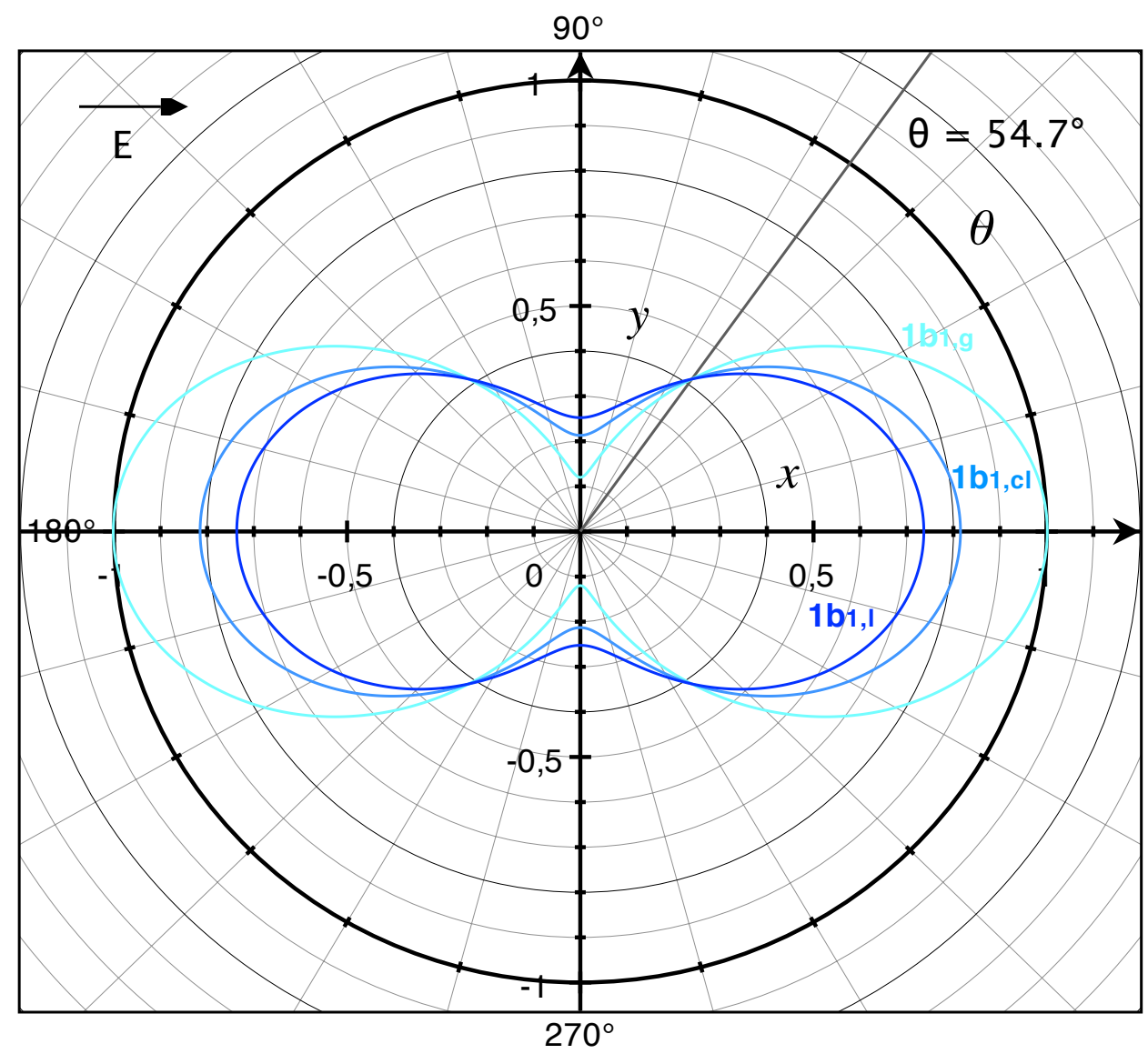

Figure 5.11: Angular distribution of photoionization for the $1 b_{1}$ orbital of water molecules in gas, small clusters and liquid phase. The same $\sigma$ is applied for all the three aggregate states in this figure, which is indicated by the same magnitudes of the three curves at the magic angle. The photoelectron distribution of the $1 b_{1}$ orbital becomes more isotropic when water molecules are more and more involved in $\mathrm{H}$-bond networks. 


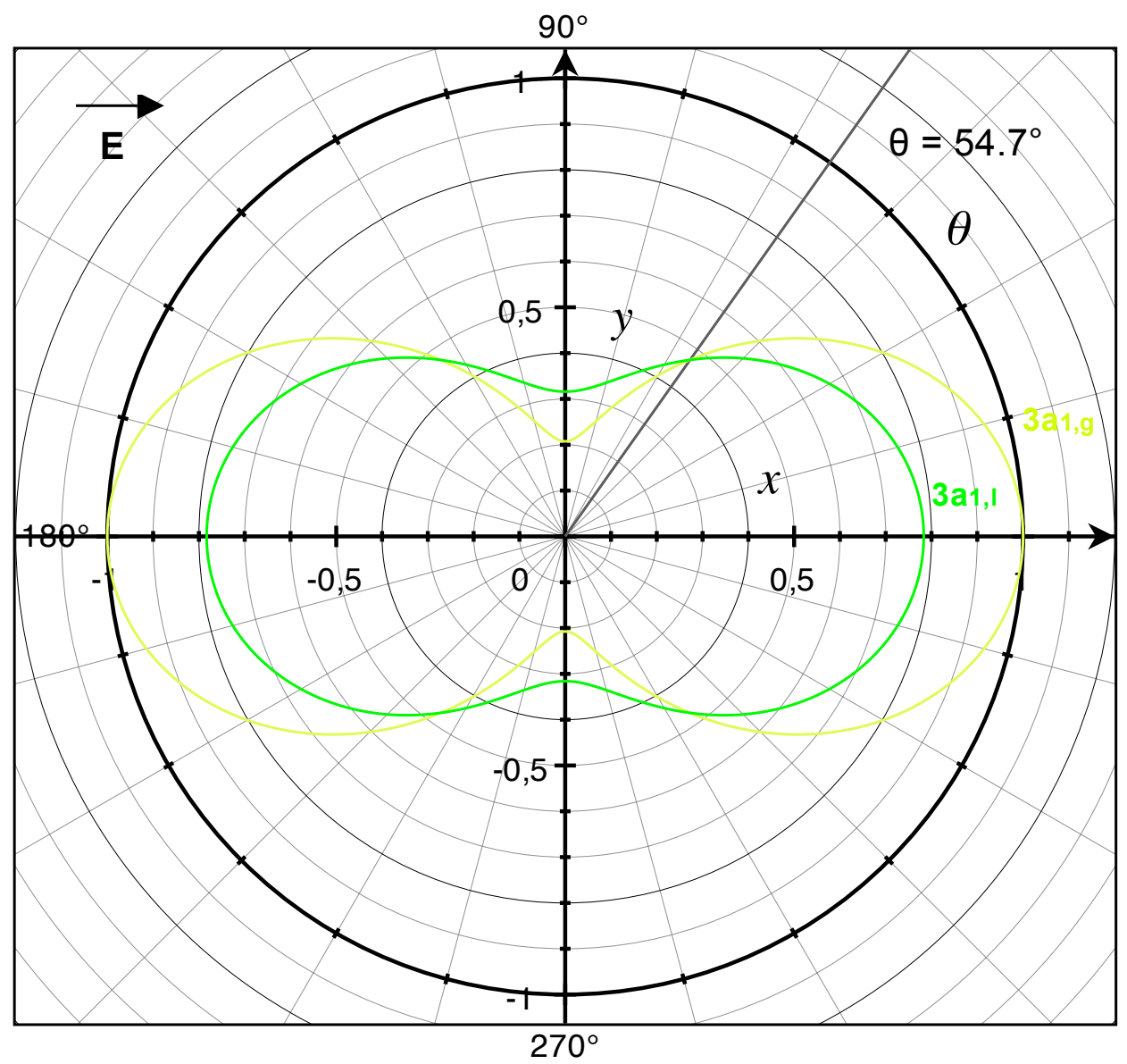

Figure 5.12: Angular distribution of photoionization for the $3 a_{1}$ orbital of water molecules in gas and liquid phase. The same $\sigma$ is applied for both of the aggregate states (notice the magic angle). The photoionization distribution of the $3 a_{1}$ orbital becomes more isotropic when water molecules are involved in H-bond networks, similar to the case of $1 b_{1}$. 


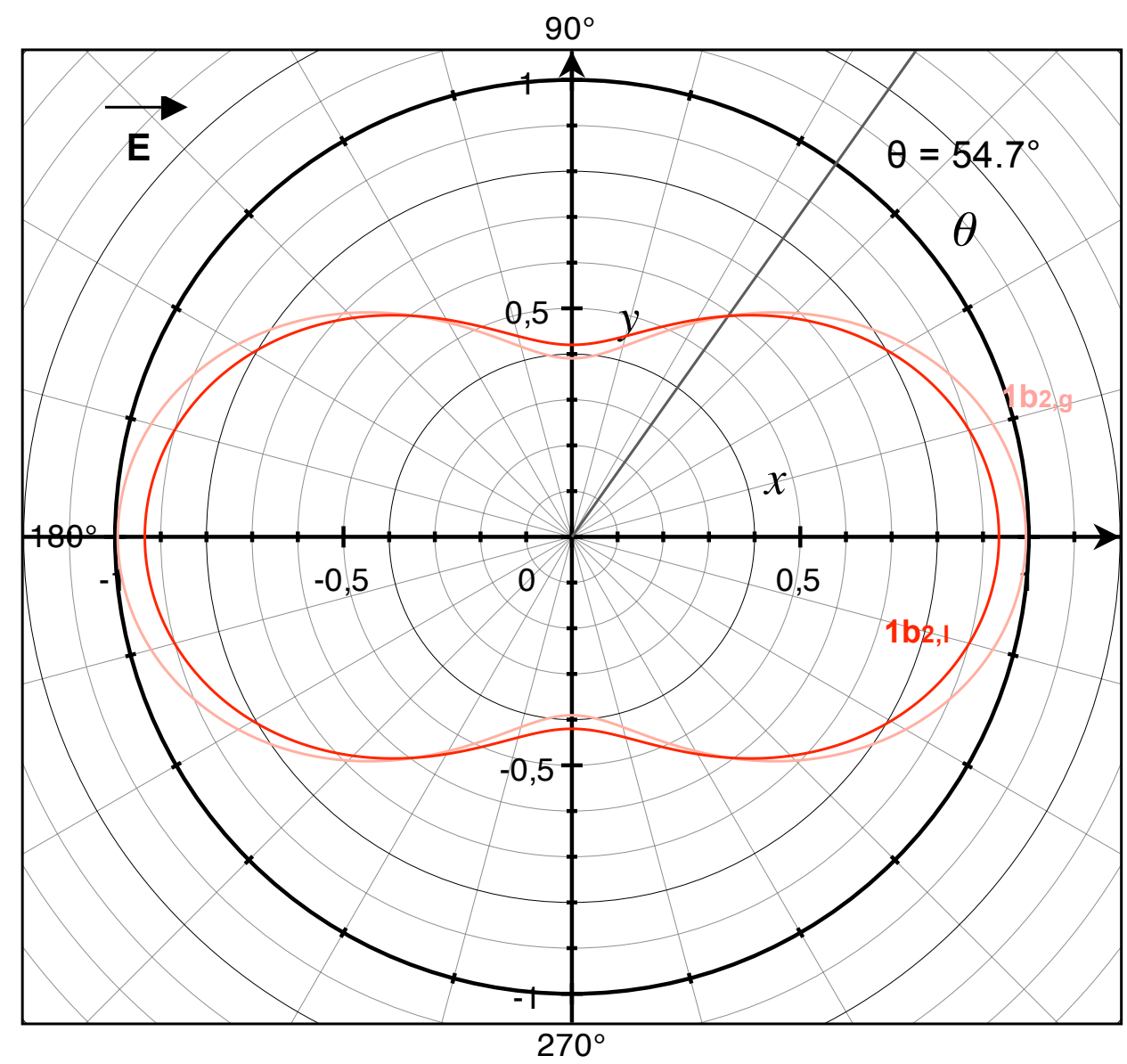

Figure 5.13: Angular distribution of photoionization for the $1 b_{2}$ orbital of water molecule in gas and liquid phase. The same $\sigma$ is used for both of the aggregate states (notice the magic angle). The photoionization distribution of the $1 b_{2}$ orbital is slightly more isotropic in the liquid than in gas phase, which points to an altered electronic nature of this orbital in contrast with the $1 b_{1}$ and $3 a_{1}$ orbitals. 
changes are more significant than for the $1 b_{2}$ orbital.

But the question is - what is the explanation for the varying anisotropy? Why are the three orbitals affected differently? To explicate these questions, we have to find out which factors can make electron emission from outer valence orbitals more isotropic, and to what extent particularly.

One of the contributions for the anisotropy changes is elastic scattering of the outgoing photoelectrons by surrounding molecules in the condensed phase. Assuming that elastic scattering is the main reason, the distribution must show an quasi-independence on kinetic energies of the photoelectrons from these three orbitals and angular distribution of the ejected electrons must be influenced to the same extent. This is yet not observed in our study, since electrons originating from $1 b_{2}$ orbital are mostly unperturbed compared with the remaining orbitals. Additionally, in small-sized clusters $(\langle n\rangle=5)$ the probability for elastic scattering is negligible. However, the angular distribution of the photoelectrons ejected from $1 b_{1, c l}$ is apparently more isotropic than $1 b_{1, g}$. All of these facts evidence that there must be other contributions changing the distribution more effectively. Another support for this presumption is the fact that the elastic scattering in liquid water is found to be weak by a recent study [74], performed on a micro-jet target as well. We suggest that the contribution from elastic scattering is equal or less than the anisotropy difference between $1 b_{2}$ orbital in both phases, which is not very large as observed.

Taking the contribution of elastic scattering into account, we expect that the increases in isotropy of the photoelectron emission distribution for $1 b_{1}$ and $3 a_{1}$ orbitals are mainly attributed to their electronic structure changes due to hydrogen bonding. In order to understand what is happening in the formation of the hydrogen bond network, the electronic structure of free water molecules will be illustrated in the first place.

Within the MO concept, the $1 b_{2}$ orbital involves bonding combinations of $\mathrm{O} 2 p$ and $\mathrm{H} 1 s$, while the $3 a_{1}$ and $1 b_{1}$ orbitals are essentially $\mathrm{O} 2 p$ non-bonding orbitals with a small share of $\mathrm{H} 1 s$ in the molecular plane and perpendicular to it, respectively (see MO diagram in Fig. 5.1). The $\beta$ value for the $1 b_{1}$ orbital in a single 
water molecule is found to be similar to that for $\mathrm{O} 2 p$ in a theoretical work by Roche et al. [106]. They attributed the small $\beta$ value of $1 b_{2}$ to its strong bonding character. From similar consideration, the decreases of the $\beta$ values of $1 b_{1}$ and $3 a_{1}$ orbitals in the liquid water may indicate that these two orbitals acquire more bonding characters by the involvement into the H-bond network, since both the $1 b_{1}$ and $3 a_{1}$ orbitals are $\mathrm{O} 2 p$-derived, as the $1 b_{2}$ orbital.

By performing ab initio MD simulations, Silvestrelli and Parrinello studied structural, electronic and bonding properties of liquid water from first principles [2, 107]. Using a novel technique, based on the generation of maximally localized Wannier functions, the molecular electronic charge distribution and its contribution to the polarization were computed quantum mechanically. Boys localized orbitals are constituted, whereby the water valence electrons are localized in two covalent orbitals lying along the $\mathrm{OH}$ bonds and two lone pair orbitals, other than within the MO concept. They found that the anisotropy of the electronic charge distribution of the water molecule is reduced in the liquid. The electronic charge becomes more spherically distributed around the oxygen-ion than in the gas phase, as a consequence of the interaction of the water molecule with the surrounding ones and the formation of hydrogen bonds. With respect to the case of the isolated molecule, in the bulk the lone pair orbitals (comparable to $1 b_{1}$ and $3 a_{1}$ ) are pulled out, while the covalent bond orbitals (comparable to $1 b_{2}$ and $2 a_{1}$ ) are pulled in. Their hypothesis that the electronic charge distribution of a H-bonded water molecule becomes more isotropic, can be partly confirmed by the results of this work. The spatial extension of the $1 b_{1}$ and $3 a_{1}$ orbitals indicates that these orbitals lose charge densities due to H-bonding. This can result in an anisotropy decrease of photoelectron emission from these two orbitals, which also means that the $\beta$ values of the $1 b_{1}$ and $3 a_{1}$ orbitals will be smaller. However, a direct evidence to the contracting of $1 b_{2}$ can not be given by our results, since the anisotropy parameter $\beta$ alters less from the gas to the liquid phase.

A comparable argument was supplied by Nilsson et al. [6]. Combining photoelectron and X-ray absorption spectroscopy with DFT calculation to consider H-bonds in ice, they found that the H-bonding involves charge transfer from $1 b_{1}$ into the $\mathrm{O}-\mathrm{H}$ anti-bonding orbitals $\left(4 a_{1}\right.$ and $\left.2 b_{2}\right)$ on neighboring molecules. At the same time, an internal $s$-p-rehybridization occurs on the oxygen atom, in order to min- 
imize the repulsive charge overlap of the connecting oxygen and hydrogen atoms. The sum of these two mechanisms results in a strong attractive electrostatic interaction. Additionally, Nilsson et al. described the internal rehybridization in another picture - orbitals overlap between two neighboring molecules in the tetrahedral arrangement inside of ice. They suggested that the $1 b_{1}$ orbital will interact primarily with the $1 b_{2}$ orbital, whereas the $3 a_{1}$ primarily interacts with the other $3 a_{1}$ and to a lesser extent with $1 b_{1}$ and $1 b_{2}$ orbitals. Although there are surely differences between the H-bonds structure in liquid and solid water, the nature and origin of the H-bond remain the same in both cases. In addition, a recent PES study on liquid water indicates that the local orbital interaction involved in hydrogen bonding in liquid water is similar to that in crystalline ice [12]. The concept of charge transfer from $1 b_{1}$ into the $\mathrm{O}-\mathrm{H}$ anti-bonding orbitals of neighboring molecules is similar to the picture of an extending lone pair orbital in [2, 107] and is further in agreement with the concept from [3] in which a HOMO-LUMO mixing in the H-bonding is interpreted as a coordinate-covalent interaction. The supposed internal rehybridization mechanism increases the internal bonding characters of all these orbitals, particularly of the $3 a_{1}$ orbital which gets more $p$ and less $s$ character. Briefly summarized, we find the following common features between the conclusion of Nilsson et al. and our results:

* Charge transfer from $1 b_{1}$ into anti-boning orbitals of an acceptor molecule and the orbital overlap or rehybridization can result in a more extended electronic distribution of the $1 b_{1}$ orbital and furthermore in a decrease of $\beta$ value in photoionization process as observed in this work.

* The increase of the bonding character of $3 a_{1}$ and $1 b_{2}$ orbitals derived by an internal rehybridization explains also the changes in angular distribution of photoelectrons emitted from these two orbitals, if a H-bond network is assembled from previous free molecules.

There is a constant dispute about which one of the $1 b_{1}, 3 a_{1}$ and $1 b_{2}$ orbitals has the most intensive involvement in H-bonding. The most common assumption is the major participation of the $3 a_{1}$ orbital. An early PE study on ice [16] showed major electronic structure changes relative to the gas-phase, including binding energy shifts and band broadening. They supposed a rather unperturbed $1 b_{1}$ orbital in 
ice and a delocalized $3 a_{1}$, associated with strong overlap between neighboring $3 a_{1}$ orbitals resulting in split bands. This was in line with early electronic structure calculations from [108]. Besides, Guo et al. used X-ray emission spectroscopy to examine the influence of the intermolecular interaction on the local electronic structure of liquid water and found a strong involvement of the $3 a_{1}$ orbital in the H-bonding [17]. To interpret the experimental results, calculations were also carried out with an ensemble of water molecules in an H-bond network, where one hydrogen bond is broken at the hydrogen site. They assumed that the $3 a_{1}$ orbital has the nodal plane perpendicular to the direction of the electric dipole moment of the water molecule and therefore can be easily polarized - electron density will be transferred from the lobe enclosing the hydrogen nuclei towards the oxygen lobe. Due to this polarization, the $3 a_{1}$ orbital mixes to a great extent with the same orbital of the neighboring molecules and loses its original character entirely.

In contrast to the studies mentioned above, there has also been the hypothesis that the $1 b_{2}$ orbital is most intensively involved in H-bonding [11]. This was based on a synchrotron PES study on a liquid water micro-jet, carried out by Winter etal.. They presented the first full-range valence photoelectron emission spectra of liquid water obtained with photon energies up to $120 \mathrm{eV}$. In particular, the partial ionization cross sections of the four valence orbitals in the liquid phase were determined for different photon energy (60, 80 and $100 \mathrm{eV}$ ). Since they did not have a suitable setup to ascertain the corresponding anisotropy parameters $\beta_{i, l}$, they assumed that the $\beta_{i, l}$ values do not differ from $\beta_{i, g}$ in the gas phase. Comparing the decrease of the photoionization cross section $\sigma_{i}$ of $1 b_{1}, 3 a_{1}$ and $1 b_{2}$, they supposed that the $1 b_{2}$ orbital experiences the environment and H-bonding most strongly. In the light of our work, we state that their supposition is probably not appropriate. The anisotropy parameter $\beta_{i}$ is much more sensitive than the cross section $\sigma_{i}$, to changes in the electronic distribution of molecular orbitals resulting from environmental variations. Although in their spectrum measured at $\theta=90^{\circ}$ and with a photon energy of $60 \mathrm{eV}$, the intensity ratios of $1 b_{1}, 3 a_{1}$ and $1 b_{2}$ bands were comparable to the results from this work, the authors came to an opposite conclusion - namely that the $1 b_{2}$ orbital is mostly involved in the H-bonding. 
In order to make a contribution to the issue about the different involvements of valence orbitals in H-bonding and conclude this part of the discussion, we have the following expectation: the $1 b_{1}$ and $3 a_{1}$ orbital of a water molecule undergo the most changes in the electron density by forming H-bond networks in the liquid phase. Therefore they show a more considerable decrease in the distribution anisotropy of photoelectrons outgoing from them. However, we do not rule out that the $1 b_{2}$ orbital also slightly participates in the formation of $\mathrm{H}$-bonds. Because, even if the anisotropy parameter $\beta$ for the $1 b_{2}$ orbital is similar for the liquid and the gas phase, any involvement in e.g. rehybridization can occur, whereby the resulting electron density distribution of the $1 b_{2}$ orbital is hardly influenced.

In the final part of this discussion, we consider the result from the angular cluster PE spectra. Many calculations have shown that the major influence of the H-bonding on the local electronic structure is due to the first coordination shell [17, 87, 109]. This confirms our experimental observation that H-bonded water molecules in small-sized clusters are more similar to molecules in the liquid, than to free water molecules in the gas phase. Consider that the elastic scattering effect in the liquid water, as discussed above, may probably reduce the $\beta$ value of $1 b_{1, l}$, while the $\beta$ value for $1 b_{1, c l}$ is not influenced. Deducing the contribution of the elastic scattering, the intrinsic anisotropy difference for these two cases is even smaller than observed in Fig. 5.11 .

A careful comparison of geometric structure or local coordination in small clusters with that in the liquid water, can also shed light on the understanding of our experimental observation. As shown in [92], the favored structure in small clusters $(n=3 \ldots 6)$ is ring-like, which means the coordination number is two per molecule. If the cluster size is larger than $n=6$, each molecule forms more than two H-bonds. Either cage-clusters or spiro-clusters can be built. With increasing average coordination, the $\mathrm{H}$-bonds increase in strength due to cooperativeness.

In the liquid phase, the majority of the water molecules are involved in a network with hydrogen bonds which break and reform due to the dynamical motion of the molecules. The average coordination number of such a network was found to be $2.4-2.6$ in an X-ray absorption examination by Mynen et al. [19], which is much lower compared to MD simulations that often give a value around 3.5 [82. In this 
XAS study, an intense, broad pre-edge feature in the XAS spectra of liquid water was associated with a large number of water molecules with asymmetric donating H-bonds (D-ASYM species). These species were identified with the help of a MD simulation with many different local structures generated from DFT calculations. Despite the disagreement with the other MD simulations, the number of nearest neighbor molecules within a radius of $3.5 \AA$ was in the range of $3-5$ molecules for all the D-ASYM species, surprisingly corresponding to the average value of 4.5 molecules in the first coordination shell determined from an integration of the radial distribution function up to the first minimum at $3.5 \AA$ [82, 83, 110]. This is due to the fact that there is a tendency to underestimate the number of broken H-bonded species using classical methods. The conclusion from the study [19] has been extended in another later study by Wernet et al. [109] which has brought about a new wave of discussion about the coordination structure in liquid water. They presented a novel concept to describe the geometric picture of water within a snapshot on a subfemtosecond time scale. "Water...consists mainly of structures with two strong H-bonds, one donating and one accepting, nonetheless implies that most molecules are arranged in strongly H-bonded chains or rings embedded in a disordered cluster network connected mainly by weak $\mathrm{H}$ bond." This hypothesis was challenged by a great deal of theoretical studies [111 113. In a recent theoretical study, Lehmann etal. approved the concept from Wernet et al. [113]. Their arguments were based on the successful reproduction of the thermodynamic properties for liquid water assuming that a major amount of twofold H-bonded and a few tetrahedrally coordinated water molecules coexist in liquid water. With the aid of the quantum cluster equilibrium method, they were able to determine the temperature-dependence of the average H-bond number. They observed a decreasing average hydrogen bond number of 2.77 at $274 \mathrm{~K}$ to 2.26 at $373 \mathrm{~K}$ and a dominance of $75 \%$ twofold hydrogen-bonded water molecules at room temperature for the mixed cluster set. This is in good agreement with the estimation $(2.4-2.6)$ by Mynen et al.

Either the concept of tetrahedral H-bond network with many D-ASYM species or the picture of a random soup (twofold H-bonded units) flecked with small "icebergs" (tetrahedrally coordinated units) is associated with low average coordination number between 2 and 3. This can also be indirectly confirmed by the results 
from the present work. As a consequence of the similar coordination number in liquid water and small clusters, the angular distribution of photoelectron emission varies not very significantly for water molecules in the bulk and in small clusters. Another evidence for the similar coordination in liquid water and small cluster is provided by the similar peak width of the $1 b_{1}$ bands both in the liquid spectra and cluster spectra (see section 5.2.2). Due to the comparable local structure in both phases, similar peak width can be expected, because the different local coordination of an individual water molecule is the main contribution to the peak broadening of $1 b_{1}$ feature. In addition, the comparison between local coordination in liquid water and small clusters also contradicts the hypothesis that the $\mathrm{H}$-bonding is no more than an electrostatic interaction or even just a dipole-dipole interaction. If this electrostatic hypothesis would be true, the effect of the surrounding molecules would be expected to be much more pronounced in the bulk water than in the small clusters, and the electronic structure of the water molecules would be very different in both cases as a consequence.

In conclusion, this study on the angular distribution of photoemission for $\mathrm{H}$ bonded water aggregate states - liquid phase and small-sized clusters provides the following results:

* For the first time, the values of the anisotropy parameters $\beta$ are determined for the three outer valence orbitals, $1 b_{1}, 3 a_{1}$ and $1 b_{2}$ in liquid water. Besides, the $\beta$ parameter for the $1 b_{1}$ orbital in small clusters $(\langle n\rangle=5)$ is also specified. These values are - without exception - smaller than those for the corresponding orbitals in free water molecules, which indicates that all of these orbitals become more extended when they undergo the phase transition from gas into denser phases.

* However, the decreases of the distribution anisotropy in the dense phases are different for the three orbitals: the $1 b_{1}$ and $3 a_{1}$ orbitals exhibit significant changes while the $1 b_{2}$ orbital varies only slightly. This points to the different involvement of the three valence orbitals in the H-bonding. $1 b_{1}$ and $3 a_{1}$ orbitals exhibit the largest changes in the electron density by form- 
ing the H-bond network in liquid phase. Accordingly, these two orbitals are expected to be strongly involved in the H-bonding.

* By comparison of the $\beta$ values for the HOMO, that is the $1 b_{1}$ orbital, in the liquid phase and in small-sized clusters, no significant difference is observed. This is assumed to be an indication for the major influence of the first coordination shell on the local electronic structure in the H-bonded network. And it is probably also an indirect evidence that the average coordination number is between 2 and 3 in the liquid water. 


\section{Chapter 6}

\section{The angular distribution of photoemission from methanol molecules, clusters and the bulk}

\subsection{Introduction}

Methanol is one of the most important organic species which has been extensively studied. In particular, liquid methanol attracts a lot of attention since it has a lot of technological and industrial applications. For instance, it is commonly used as organic solvent in protein investigations [114] and more recently, it is used as an important fuel alternative [115. Liquid methanol has many unusual physical properties: it is stable over a wide range of temperatures, has a small viscosity coefficient, even smaller than liquid water, and an anomalous vaporization enthalpy.

Methanol molecule has the chemical formula $\mathrm{CH}_{3} \mathrm{OH}$. The main electron density of the $\mathrm{OH}$ bond is accumulated on the oxygen atom, because of its high electronegativity. Also the methyl group donates electron density to the oxygen atom, which weakens the polarization of the $\mathrm{OH}$ bond. The $\mathrm{CH}_{3} \mathrm{OH}$ molecule has $\mathrm{C}_{s}$ symmetry and the electronic structure in the ground state can be expressed as

$$
\left(1 a^{\prime}\right)^{2}\left(2 a^{\prime}\right)^{2}\left(3 a^{\prime}\right)^{2}\left(4 a^{\prime}\right)^{2}\left(5 a^{\prime}\right)^{2}\left(1 a^{\prime \prime}\right)^{2}\left(6 a^{\prime}\right)^{2}\left(7 a^{\prime}\right)^{2}\left(2 a^{\prime \prime}\right)^{2} .
$$

The outermost five MOs result from the hybridization of $p$ orbitals of carbon and oxygen atoms. The highest occupied molecular orbital (HOMO) $2 a^{\prime \prime}$ has mainly 
$\mathrm{O} p$ character with a small share of $\mathrm{C} p$ character. The HOMO is constructed mainly by the out-of-the-plane $p$ orbital of carbon and oxygen atoms. The electron density of this orbital is mostly localized on the oxygen atom. $5 a^{\prime}, 7 a^{\prime}$ and $2 a^{\prime \prime}$ orbitals are more oxygen-like while $1 a^{\prime \prime}$ and $6 a^{\prime}$ orbitals are more carbon-like.

Hydrogen bonding in methanol is different from that in water due to the existence of the sterical methyl group. In methanol dimer, the length of the hydrogen bond is found to be $1.96 \AA$, slightly longer than that in water dimer $(1.95 \AA)$ [116, 117]. The interaction between the two methanol molecules in the dimer is dominated by the pure electrostatic interaction. In a larger assemble of $\mathrm{H}$-bonded methanol molecules, the nature of the H-bonding can be different, due to cooperative effect of H-bonds. Each methanol molecule can build a maximum of two H-bonds, as donor or acceptor, respectively. As a consequence, no threedimensional network can be formed as in water. Only chains or rings are possible. Due to the steric effect of the methyl group, a chain consisting of several methanol molecules can not be a straight line. This entire chain is strongly polarized, because the electron density is "pushed" even further forwards along the chain, from one end of the chain to the other. In contrast, such polarization does not exist in the ring structure, since the donating and accepting of the electron densities is balanced among the molecules. This difference between chain and ring structures will result in a distinct variation of their electronic structures.

Many studies have been carried out to examine the electronic structure of methanol, via diverse electronic spectroscopic methods. However, the general attention is paid to revealing the geometric structures of methanol. In the crystalline phase, it has been found that methanol molecules form long one-dimensional chains of H-bonds [118]. The picture in liquid phase is not yet clear. Several numerical simulations [119, 120] and neutron diffraction experiments [121, 122] have indicated that the chain-like structure is also found in liquid methanol, while many other experiments pointed to the existence of cyclic clusters [117, 123, 124]. The hypothesis of a cyclic structure was supposed by Pauling [125]. According to an energetic aspect, Pauling argued that the favored structures in liquid methanol are cyclic hexamer species. Some results from neutron diffraction [123] and X-ray scattering [124] confirmed the presence of cyclic hexamers. Also the geometric structure of methanol clusters has been studied. Small clusters (oligomers) are found to 
prefer cyclic structures, referring to many theoretical investigations [126, 127] and experimental observations [128, 129]. In contrast, medium-sized methanol clusters are found to have long chains as dominating structures [127].

An extensive study on the electronic structure of liquid methanol was reported in detail in [117]. The electronic difference between chain and ring structures was monitored by an X-ray emission study combined with a DFT calculation [117]. They expected that liquid methanol is a mixture of ring and chain structures, which have predominantly six and eight methanol units. Besides, the molecular orbitals of the ring structure with six methanol units were found to show notable similarity to the covalent $\pi$ orbitals of the benzene ring, which indicates that the hydrogen bonding in the ring structures have significant covalent contributions. They suggested that the methanol rings may be the best example to illustrate the covalent contribution to the H-bonding.

For our PE investigation of methanol, the general motivation is to compare differently $\mathrm{H}$-bonded model systems, in order to understand the inimitability of the hydrogen bonding in liquid water. By tracing the variation of the electronic structure of molecular orbitals, precious information can be obtained about the influence of the H-bonding on the orbital picture. As already mentioned in the last chapter, the determination of the anisotropy parameter of a valence orbital, HOMO particularly, offers a useful tool for examining the change of electron density within the affected orbital. The major goal of this work is not to answer the question how the geometric structure looks like in the liquid methanol or clusters. However, within the present investigation there might be some indications for a qualitative estimation of the coexistence of both ring and chain structures.

\subsection{Angle-dependent photoelectron spectra}

In this section, all the presented angular photoelectron spectra from liquid methanol are measured using the similar experimental approach which is described in the last chapter. Also a nozzle with an opening diameter of $16 \mu \mathrm{m}$ is applied to generate the methanol liquid micro-jet. In order to eliminate charging effects on the liquid jet, a $0.005 \mathrm{~mol} / l \mathrm{NaCl}$ methanol solution is used as sample. 
For the angular measurement of the PE spectra from methanol cluster, we use both the same cluster source and the nozzle, described in subsection 4.5 , however upon another condition. Taking the thermodynamic properties of methanol into account, the reservoir and nozzle temperatures are adjusted to be $40^{\circ} \mathrm{C}$ and $58^{\circ} \mathrm{C}$ respectively, in order to generate small methanol clusters. To characterize clusters produced under this stagnation condition, we do not prefer the scaling laws method, which has been demonstrated in section 4.5. Initially for rare gases, Hagena introduced empiric parameters to describe the degree of condensation in a certain system [76, 77]. Although a similar approach has been successfully made for clusters consisting of small molecules e.g. water, the application of scaling law for methanol clusters generated in our experiment is not really appropriate. This is due to the fact that the characteristic parameters have not been empirically determined and not proved further. Moreover, as shown in the application for estimating the mean size of the water clusters generated in this experiment, a variance of a factor 2 can be found, particularly for very small clusters. Hence, to identify the cluster size of methanol, we refer to a previous PE study of free neutral methanol clusters [130]. This will be demonstrated after the presentation of the PE spectra from both methanol liquid and cluster.

In Fig. 6.1, three PE spectra from different aggregate states of methanol are presented, in order to demonstrate their varying features. The attribution of these spectra also is illustrated by associating different bands with the corresponding orbitals. There are two notable differences between these spectra - the binding energy shift of the HOMO $2 a^{\prime \prime}$, and the different peak width for this orbital in the clusters and liquid phase. The binding energies of the $2 a^{\prime \prime}$ orbital are as follows: $11.0 \mathrm{eV}$ (gas), $10.4 \mathrm{eV}$ (clusters) and $9.7 \mathrm{eV}$ (liquid). These result in binding energy shifts of $0.6 \mathrm{eV}$ (gas-to-cluster) and $1.3 \mathrm{eV}$ (gas-to-liquid), which are comparable with those values for water. However, the FWHM of $2 a_{l}^{\prime \prime}$ and $2 a_{c l}^{\prime \prime}$ peaks are $1.2 \mathrm{eV}$ and $0.7 \mathrm{eV}$ respectively, which is dissimilar to water. The peak width is nearly identical for HOMO $1 b_{1}$ both in the liquid and in small clusters. Considering the causes for the peak broadening (see section 5.2 ), the different peak width in both cases can be associated with the different local geometric structures in these aggregate states. In other words, methanol molecules are more similarly involved in the cluster environment than in the liquid phase. There must be a larger variation 


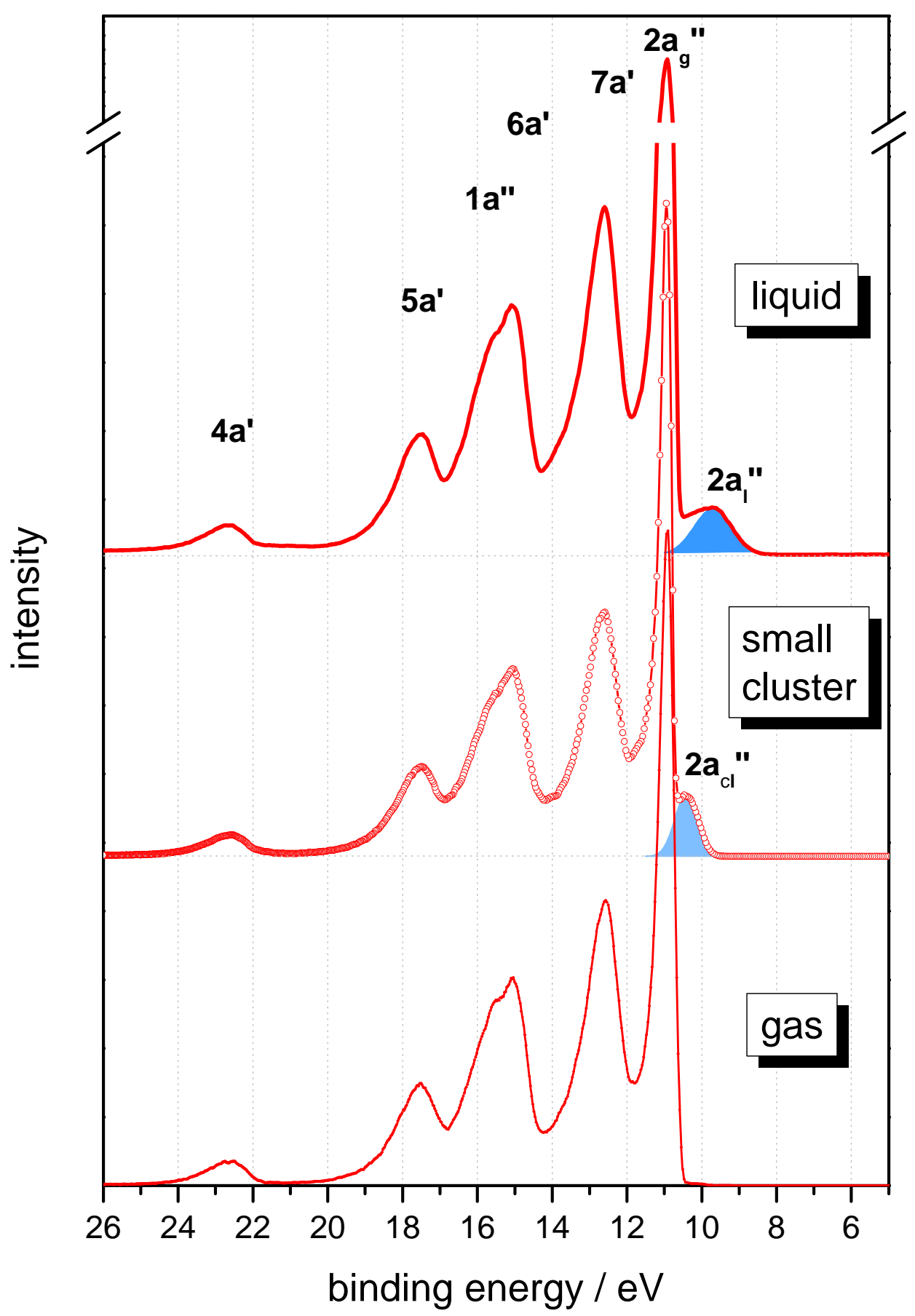

Figure 6.1: PE spectra from methanol molecules, small clusters and liquid jet measured with $p$-polarized EUV light. Notice the binding energy shift of $2 a^{\prime \prime}$ orbital from gas phase, via cluster to liquid phase. The FWHM of $2 a_{l}^{\prime \prime}$ and $2 a_{c l}^{\prime \prime}$ peaks are $1.2 \mathrm{eV}$ and $0.7 \mathrm{eV}$, respectively. 
of individual molecules in the liquid methanol than in small clusters. This may be an indication for revealing the possible geometric structures in both aggregate states, which will be discussed later in section 6.3.

There is still an open question about the average size of the methanol clusters probed in this experiment. In the PE study reported in [130], methanol clusters were produced via adiabatic expansion, under different stagnation conditions, which is comparable to our experimental approach. The cluster size was characterized by using photoelectron spectroscopy and line shape modeling. It was found that clusters of two distinct categories - small and medium-sized clusters - could be produced by changing the stagnation pressure. In the ultraviolet photoelectron spectra, both the gas-to-cluster binding energy shift and peak width of the small cluster regime are the same as in our spectra. Additionally, related $\mathrm{X}$-ray photoelectron spectra were measured in their experiment. The calculation of the $\mathrm{C} 1 s$ ionization potentials for various structured oligomers indicated that the small-sized clusters are mostly cyclic oligomers $\left(\mathrm{CH}_{3} \mathrm{OH}\right)_{n}$ with $n<10$. Referring to this study, we suppose that chiefly small cyclic methanol clusters $(n<10)$ are generated upon our experimental condition.

In Fig. 6.2 and 6.3, the angular PE spectra from methanol micro-jet and small clusters are presented. Assignment of bands in the PE spectra from methanol in condensed phases are more challenging because of band overlap. Moreover, the subtracting procedure used in section 5.2.1 is not satisfactory for determining pure liquid or cluster spectra of methanol, due to the relatively high contribution from isolated methanol molecules to the mixed jet or cluster spectra. Hence, only the $2 a_{g}^{\prime \prime}, 2 a_{l}^{\prime \prime}$ and $2 a_{c l}^{\prime \prime}$ features are taken into consideration to examine the partial change of the electronic structure resulting from the condensation. This is comparable to the procedure used for water clusters (see section 5.2.2). In Fig.6.2 or Fig 6.3 , different angular distribution properties are visible for $2 a_{g}^{\prime \prime}$ and $2 a_{l}^{\prime \prime}$ or $2 a_{c l}^{\prime \prime}$. The spectra in both figures are scaled so that the $2 a_{g}^{\prime \prime}$-peaks exhibit the similar intensity for all polarization directions. Both the $2 a_{l}^{\prime \prime}$ - and $2 a_{c l}^{\prime \prime}$-peaks have their strongest intensity in connection with $s$-polarized EUV light. However, the relative intensities of the $2 a_{l}^{\prime \prime}$ - and $2 a_{c l}^{\prime \prime}$-peaks are apparently not the same, which indicates that the angular distribution of the photoelectron ejected from these orbitals are 
different. The complete understanding of these experimental observations can be achieved only if the angular distribution of the photoemission is explicated for $2 a^{\prime \prime}$ orbital in methanol liquid and small clusters, by determining the related anisotropy parameter $\beta$.

\subsection{Results and discussion}

The procedure for the determination of the $\beta$ parameter has been demonstrated in the section 5.3 and the complication of this method has also been illustrated for the

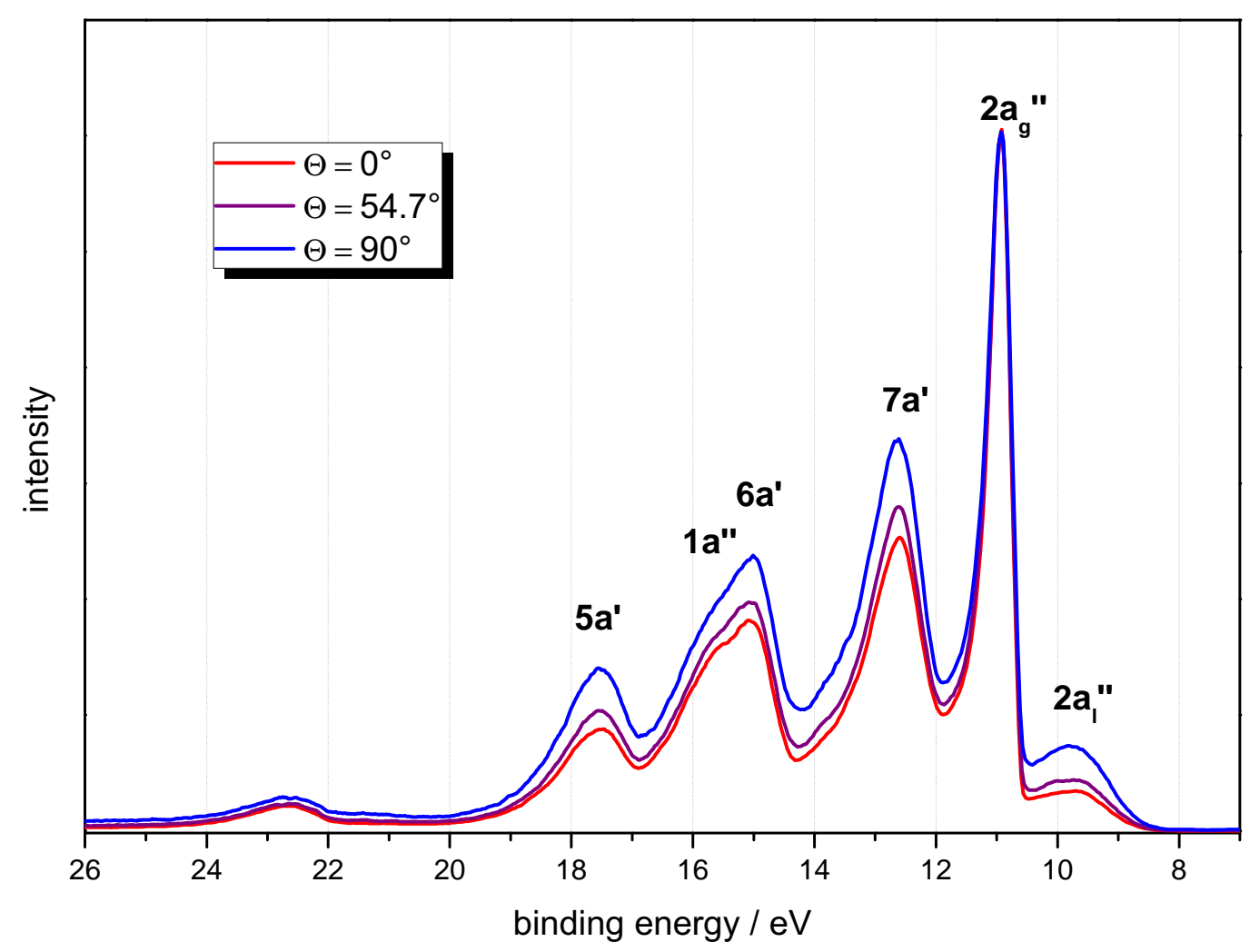

Figure 6.2: PE spectra from methanol liquid jet measured at different angles $\theta$. The photoelectron counts of those spectra are scaled so that the $2 a_{g}^{\prime \prime}$-peaks are similar. Notice the different intensities in the range of $2 a_{l}^{\prime \prime}$. 


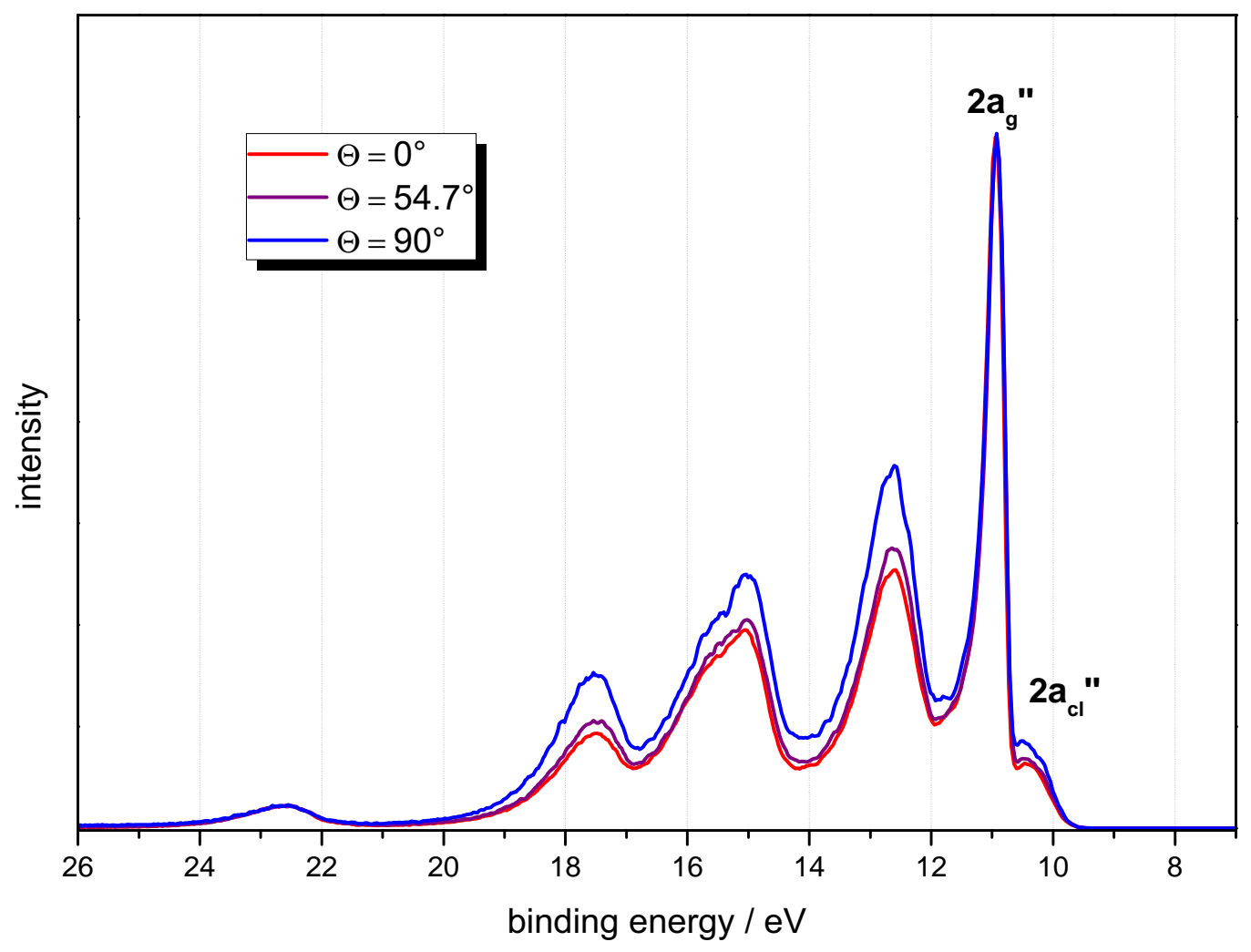

Figure 6.3: PE spectra from small methanol clusters measured at different angles $\theta$. The photoelectron counts of those spectra are scaled so that the $2 a_{g}^{\prime \prime}$-peaks are similar. Notice the different intensities in the range of $2 a_{c l}^{\prime \prime}$.

case of water (see section 5.4). Here, only the results of $\beta$ values for the HOMOs $2 a_{g}^{\prime \prime}, 2 a_{l}^{\prime \prime}$ and $2 a_{c l}^{\prime \prime}$ will be presented in Tab.6.1. Analog to the case of water, the same photoionization cross section $\sigma$ is used to depict the angular distribution of photoemission for the HOMO $2 a^{\prime \prime}$ in the three aggregate states (see Fig.6.4). This is due to the similar approximation that the $\sigma$ value is slightly influenced by intermolecular interaction, in contrast to the $\beta$ parameter (see section 5.4).

In Fig. 6.4, the following trend is also observed in the case of water - the angular distribution of photoemission from the HOMO orbital becomes more and more isotropic. However, comparing the Fig.6.4 with Fig. 5.11, apparent distinc- 


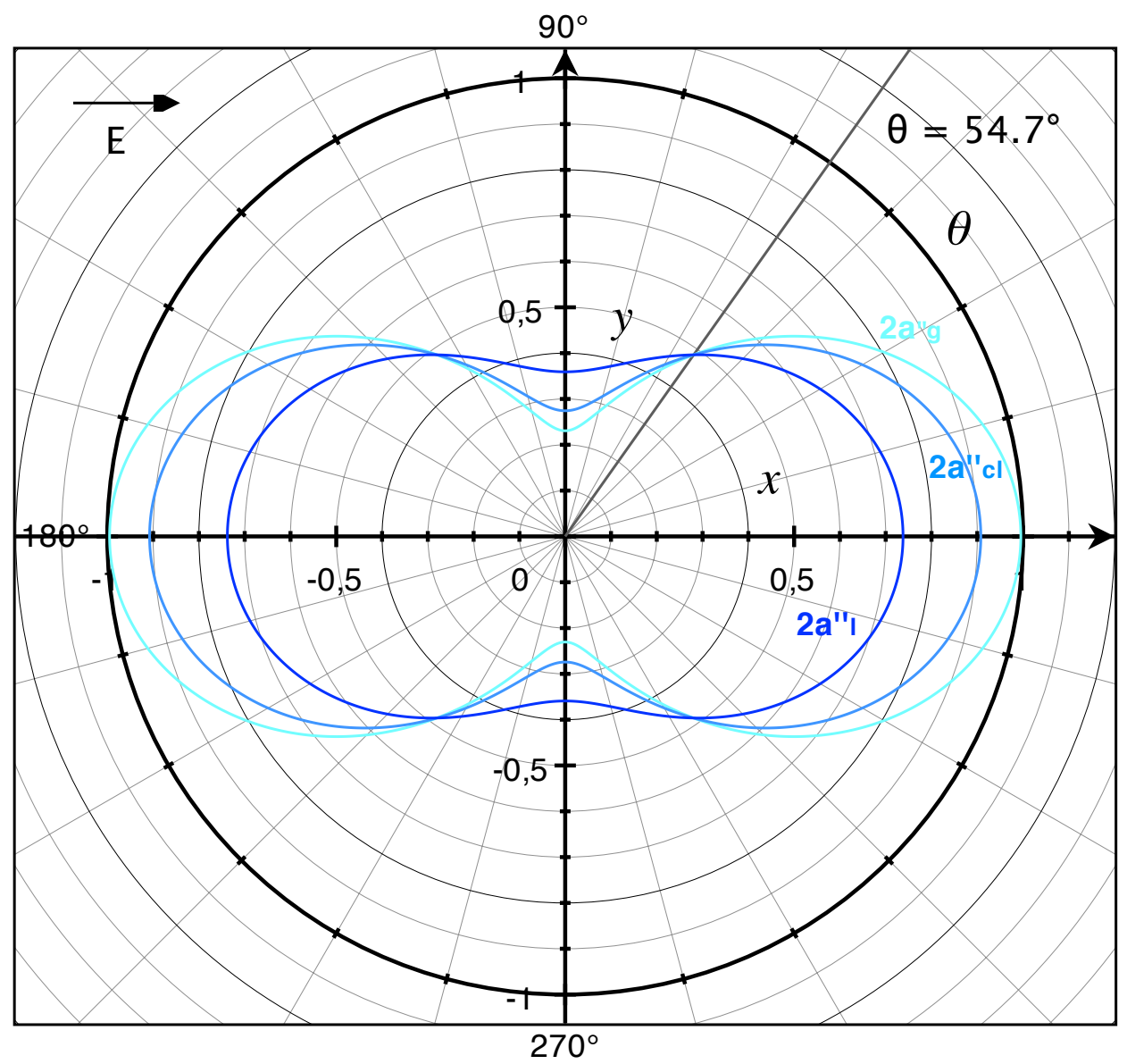

Figure 6.4: Angular distribution of photoionization for $2 a^{\prime \prime}$ orbital of methanol molecule in gas, small clusters and liquid phase. The same $\sigma$ is applied for all the three aggregate states in this figure, which is indicated by the same magnitudes of the three curves at the magic angle. The photoionization distribution of $2 a^{\prime \prime}$ orbital becomes more isotropic from gas, via small cluster to liquid phase. 


\begin{tabular}{llll}
\hline$h \nu=38.7 e V$ & \multicolumn{3}{c}{ HOMO } \\
\cline { 2 - 4 } & $2 a_{g}^{\prime \prime}$ & $2 a_{c l}^{\prime \prime}$ & $2 a_{l}^{\prime \prime}$ \\
\hline$\beta$ value & 1.1 & 0.9 & 0.5 \\
\hline
\end{tabular}

Table 6.1: Experimental results for PE angular distribution $(h \nu=38.7 \mathrm{eV})$ for methanol gas, small clusters and liquid. The error of the $\beta_{i}$ values is estimated to be $10 \%$.

tion between them can be assessed, according to evolutions of the photoemission anisotropy for these two HOMOs. The $\beta$ value for $1 b_{1}$ orbital decreases firstly significantly from $\mathbf{1 . 4}$ in gas phase to $\mathbf{1 . 0}$ in small clusters, and then slightly to $\mathbf{0 . 8}$ in the liquid. This evolution is further more clearly demonstrated by inspecting the curve shapes in Fig.5.11. In contrast, the $\beta$ value for $2 a^{\prime \prime}$ orbital reduces at first slightly from $\mathbf{1 . 1}$ (gas phase) to $\mathbf{0 . 9}$ (small clusters), and then considerably to $\mathbf{0 . 5}$ (liquid phase). Furthermore, this observation - the phase-dependent progression of $\beta$ value are different for HOMOs of water and methanol molecules may be connected with the other above-mentioned fact: The width of the $2 a^{\prime \prime}$ peak is different in the spectra from methanol liquid $(F W H M=1.2 \mathrm{eV})$ and cluster $(\mathrm{FWHM}=0.7 \mathrm{eV})$, while the $1 b_{1}$-peak retains its width in both two phases.

To explain these two dissimilarities, following hypothesis is made. In the liquid methanol, chain- and ring-like units of molecules coexist resulting from $\mathrm{H}$ bonding [117]. By contrast, ring-like oligomers dominate in small methanol clusters [127]. As a consequence, the local structures are more various in the liquid methanol than in small clusters. A mixture from ring and chain structures results in a broader spectral feature, because of the distinct electronic structure difference of these dissimilar geometric structures. Nevertheless, the formation of collective H-bonds is one-dimensional in both chain and ring structures due to the existence of the methyl group. In condensed water aggregates, however, the forming of the H-bond network extends in three dimensions. The overlap of the molecular orbitals in such a network is inevitable and strong, because the water molecules are ordered very tightly. In addition, the small size of water molecule and its affinity for building more than two H-bonds result in the crucial influence of the first co- 
ordination shell on the electronic structure of water [109]. In H-bonded methanol molecule ensembles, the connection of molecules is not extending, but rather fractional in a snapshot of the MD picture. This leads to the increasing influence of the non-direct environment, meaning the surrounding molecules from the second, third or even further coordination shells. As a consequence, the $\beta$ value for $1 b_{1, c l}$ is similar to $\beta$ value for $1 b_{1, l}$, rather than $\beta$ for $1 b_{1, g}$. Furthermore, it can be expected that the electronic structures of water molecules in small clusters $(n<10)$ are generally more similar to those in the liquid water, than to those of free water molecules. In a ring unit of methanol molecules, the interaction within the ring may be stronger than along a chain unit. But molecules inside a ring unit can still be influenced by molecules not H-bonded to the ring structure. Due to this concurrence of the non-H-bonded molecules, the electronic structure of a particular ring molecule "senses" their influence more and more, and changes continuously with the increasing size of the H-bonded molecule ensemble. This is the reason for the gradual evolution of the $\beta$ value with increasing density.

As a conclusion, the hydrogen bonding in liquid water or small clusters is considerable different from the other weaker models, such as methanol. The H-bonds in water are more powerful and intensive so that the electronic structures of the $\mathrm{H}$-bonded water molecules are immediately affected even when only small cluster units are formed. In the case of methanol, it is quite different. Due to the comparatively weak influence of the directly connected H-bonds on the molecular electron density, the molecules from the second, third and further coordination shells have more influence on the electronic structure of a central molecule. Therefore, its electronic structure changes gradually with increasing number of surrounding molecules. 



\section{List of Figures}

$2.1 \quad$ Sketch illustrating the mechanism behind PES. . . . . . . . . . . . 9

2.2 Illustration depicting the principle of PES experiments. . . . . . . . 10

2.3 Angular distribution of photoelectron emission of a single atom. . . 14

2.4 Angular distribution of photoionization associated with $\beta=2,1,0,-1$. 17

3.1 Semiclassical three-step model for high harmonic generation. . . . . 21

3.2 Schematic spectrum of the high harmonics generated in our experiment. . . . . . . . . . . . . . . . . 23

3.3 High harmonic generation by focussing the $800 \mathrm{~nm}$ fundamental light pulses into argon gas. . . . . . . . . . . . . . . . . 25

3.4 CCD camera image of the $25^{\text {th }}$ harmonic. . . . . . . . . . . . 25

3.5 The SEM image of a nano-structure used in this work. . . . . . . . 28

3.6 Optical Setup of VENTEON 1 PULSE : ONE. . . . . . . . . . . . 30

3.7 Experimental setup of HHG exploiting nano-structure. . . . . . . . 32

3.8 One picture (100 ms integration time) from the movie sequence recorded during a measurement. Sample: GA19-4. . . . . . . . . . . 32

3.9 HHG efficiency dependence on the Ar pre-pressure. . . . . . . . . . . 33

3.10 Evidence for the successful HHG in the presence of our nano-structure. 34

3.11 Temporal trend of the damage process on the nano-structure. . . . 35

4.1 The laser beam line for the PES experiment. . . . . . . . . . . . . . 39

4.2 Sketch of the multi-pass amplification unit. . . . . . . . . . . . . . . 40

4.3 The Vacuum setup. . . . . . . . . . . . . . . . . . . . . . 43

4.4 Picture of the liquid micro-jet. . . . . . . . . . . . . . . . . . . . . . . . . . . . . 45

4.5 Sketch of the cluster source. . . . . . . . . . . . . . . . . . . . . 47

4.6 Sketch of the TOF photoelectron spectrometer. . . . . . . . . . . 48 
4.7 A transformed spectrum of water vapor. . . . . . . . . . . . . 50

5.1 The $\mathrm{MO}$ diagram of $\mathrm{H}_{2} \mathrm{O}$ molecule. . . . . . . . . . . . . . . . . 53

5.2 Photoelectron spectrum measured on a water micro-jet with $p$ polarized EUV light $\left(\theta=0^{\circ}\right) . \ldots \ldots$. . . . . . . . . 60

5.3 Sketch of the different approaches for measuring gas and jet spectra. 61

5.4 Polarization-dependent PE spectra from a liquid water micro-jet. . 62

5.5 Liquid PE spectra at different angles $\theta$. . . . . . . . . . . . . 67

$5.6 \quad$ Rescaled liquid spectra at different angles $\theta$. . . . . . . . . . . . . 68

$5.7 \quad$ Photoelectron spectra of water molecules, small water clusters and liquid water measured with $p$-polarized EUV light. . . . . . . . . . . 69

$5.8 \quad$ Photoelectron spectra of small water clusters measured with differently polarized EUV light. . . . . . . . . . . . . . . . 72

5.9 Angular distribution of photoionization for $1 b_{1}, 3 a_{1}$ and $1 b_{2}$ orbitals of gaseous water. . . . . . . . . . . . . . . . . . 79

5.10 Angular distribution of photoelectrons from the $1 b_{1}, 3 a_{1}$ and $1 b_{2}$ orbitals of liquid water. . . . . . . . . . . . . . 80

5.11 Angular distribution of photoionization for the $1 b_{1}$ orbital of water molecules in gas phase, small clusters and liquid phase. . . . . . . . 82

5.12 Angular distribution of photoionization for the $3 a_{1}$ orbital of water molecules in the gas and liquid phase. . . . . . . . . . . . . 83

5.13 Angular distribution of photoionization for the $1 b_{2}$ orbital of water molecule in the gas and liquid phase. . . . . . . . . . . . . . . . 84

6.1 PE spectra from methanol molecules, small clusters and liquid jet measured with $p$-polarized EUV light. . . . . . . . . . . . . . . . . 97

$6.2 \quad$ PE spectra from methanol liquid jet measured at different angles $\theta .99$

6.3 PE spectra from small methanol clusters measured at different an-

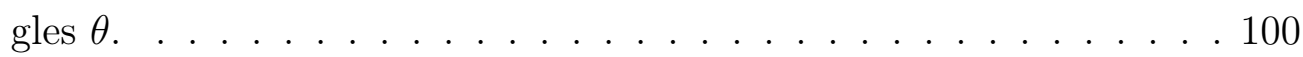

6.4 Angular distribution of photoionization for $2 a^{\prime \prime}$ orbital of methanol molecule in gas phase, small clusters and liquid phase. . . . . . . . . 101 


\section{List of Tables}

\begin{tabular}{|lll}
\hline 5.1 & Experimental results for PE angular distribution $(h \nu=38.7 \mathrm{eV})$ of \\
\hline & free water molecule, small water clusters and liquid water. $\ldots .$. & 77
\end{tabular}

$6.1 \quad$ Experimental results for PE angular distribution $(h \nu=38.7 \mathrm{eV})$ of free methanol molecule, small methanol clusters and liquid methanol.102 



\section{Bibliography}

[1] P. Ball, "Water - an enduring mystery", Nature, 452 (7185), 291, 2008.

[2] P. Sivestrelli and M. Parrinello, "Water molecule dipole in the gas and in the liquid phase", Phys. Rev. Lett., 82 (16), 3308, 1999.

[3] T. K. Ghanty, V. N. Staroverov, P. R. Koren, and E. R. Davidson, "Is the hydrogen bond in water dimer and ice covalent?", J. Am. Chem. Soc., 122 (6), 1210, 2000.

[4] E. D. Isaacs, A. Shukla, P. M. Platzman, D. R. Hamann, B. Barbiellini, and C. A. Tulk, "Compton scattering evidence for covalency of the hydrogen bond in ice", J. Phys. Chem. Solids, 61 (3), 403, 2000.

[5] B. Barbiellini and A. Shukla, "Ab initio calculations of the hydrogen bond", Phys. Rev. B, 66 (23), 235101, 2002.

[6] A. Nilsson, H. Ogasawara, M. Cavalleri, D. Nordlund, M. Nyberg, P. Wernet, and L. G. M. Pettersson, "The hydrogen bond in ice probed by soft Xray spectroscopy and density functional theory", J. Chem. Phys., 122 (15), 154505, 2005.

[7] M. J. Campbell, J. Liesegang, J. D. Riley, R. C. G. Leckey, and J. G. Jenkin, "The electronic structure of the valence bands of solid $\mathrm{NH}_{3}$ and $\mathrm{H}_{2} \mathrm{O}$ studied by ultraviolet photoelectron spectroscopy", J. Electron. Spectrosc. Relat. Phenom., 15 (1), 83, 1979.

[8] O. Link, E. Lugovoy, E. Vohringer-Martinez, Y. Liu, K. Siefermann, M. Faubel, H. Grubmüller, R. B. Gerber, Y. Miller, and B. Abel, "Ultrafast phase transitions in metastable water near liquid interfaces", Faraday Discuss. Chem. Soc., 141, 67, 2008. 
[9] O. Link, E. Lugovoy, K. Siefermann, Y. Liu, M. Faubel, and B. Abel, "Ultrafast electronic spectroscopy for chemical analysis near liquid water interfaces: concepts and applications", Appl. Phys. A, 96 (1), 117, 2009.

[10] M. Faubel, B. Steiner, and J. P. Toennies, "Photoelectron spectroscopy of liquid water, some alcohols, and pure nonane in free micro jets", J. Chem. Phys., 106 (22), 9013, 1997.

[11] B. Winter, R. Weber, W. Widdra, M. Dittmar, M. Faubel, and I. V. Hertel, "Full valence band photoemission from liquid water using EUV synchrotron radiation", J. Phys. Chem. A, 108, 2625, 2004.

[12] D. Nordlund, M. Odelius, H. Bluhm, H. Ogasawara, L. Pettersson, and A. Nilsson, "Electronic structure effects in liquid water studied by photoelectron spectroscopy and density functional theory", Chem. Phys. Lett., 460, 86, 2008.

[13] S. Barth, M. Oncak, V. Ulrich, M. Mucke, T. Lischke, P. Slavicek, and U. Hergenhahn, "Valence ionization of water clusters: From isolated molecules to bulk", J. Phys. Chem. A, 113, 13519, 2009.

[14] H. Siegbahn, "Electron spectroscopy for chemical analysis of liquids and solutions", J. Phys. Chem., 89, 897, 1985.

[15] M. Lundholm, H. Siegbahn, S. Holmberg, and M. Arbman, "Core electron spectroscopy of water solutions", J. Electron. Spectrosc. Relat. Phenom., 40, $163,1986$.

[16] N. Mårtensson, "Molecular and solid water, a comparative ESCA study.", New J. Chem., 1, 191, 1977.

[17] J.-H. Guo, Y. Luo, A. Augustsson, J.-E. Rubensson, H. r. C. Såthe, H. Siegbahn, and J. Nordgren, "X-ray emission spectroscopy of hydrogen bonding and electronic structure of liquid water", Phys. Rev. Lett., 89 (13), 137402, 2002. 
[18] B. Winter, E. F. Aziz, U. Hergenhahn, M. Faubel, and I. Hertel, "Hydrogen bonds in liquid water studied by photoelectron spectroscopy", J. Chem. Phys., 126, 124504, 2007.

[19] S. Myneni, Y. Luo, L. Å. Näslund, M. Cavalleri, L. Ojamäe, H. Ogasawara, A. Pelmenschikov, P. Wernet, P. Väterlein, C. Heske, Z. Hussain, L. G. M. Pettersson, and A. Nilsson, "Spectroscopic probing of local hydrogen-bonding structures in liquid water", J. Phys. Condens. Matter, 14, L213, 2002.

[20] C. D. C. J. D. Smith, K. R. Wilson, B. M. Messer, R. C. Cohen, and R. J. Saykally, "Energetics of hydrogen bond network rearrangements in liquid water", Science, 306 (5697), 851, 2004.

[21] O. Björneholm, F. Federmann, S. Kakar, and T. Möller, "Between vapor and ice: Free water clusters studied by core level spectroscopy", J. Chem. Phys., 111 (2), 546, 1999.

[22] H. Hertz, "Über einen Einfluss des ultravioletten Lichtes auf die electrische Entladung", Ann. Phys., 267 (8), 983, 1887.

[23] A. Einstein, "Über einen die Erzeugung und Verwandlung des Lichtes betreffenden heuristischen Gesichtspunkt", Ann. Phys., 322 (6), 132, 1905.

[24] S. Hüfner, Photoelectron spectroscopy, Springer-Verlag, Berlin, 2003.

[25] C. R. Brundle and D. W. Turner, "High resolution molecular photoelectron spectroscopy. II. Water and deuterium oxide", Proc. R. Soc. London, Ser. A, 307 (1488), 27, 1968.

[26] K. Siegbahn, "Electron spectroscopy for chemical analysis (E.S.C.A.)", Philos. Trans. R. Soc. London, Ser. A, 268 (1184), 33, 1970.

[27] M. Drescher, "Time-resolved ESCA: a novel probe for chemical dynamics", Z. Phys. Chem., 218 (11), 1147, 2004.

[28] H. Fellner-Feldegg, H. Siegbahn, L. Asplund, P. Kelfve, and K. Siegbahn, "ESCA applied to liquids IV. A wire system for ESCA measurements on liquids", J. Electron. Spectrosc. Relat. Phenom., 7, 421, 1975. 
[29] M. Faubel, S. Schlemmer, and J. P. Toennies, "A molecular beam study of the evaporation of water from a liquid jet", Z. Phys. D, 10, 269, 1988.

[30] H. A. Bethe, Handbuch der Physik, vol. 24, Springer-Verlag, Berlin, 1938.

[31] M. Peshkin, "Angular distributions of photoelectrons: Consequences of symmetry", in Adv. Chem. Phys., vol. 18, pp. 1-14, John Wiley \& Sons, Inc., Hoboken, NJ, USA, 1970.

[32] J. Cooper and R. N. Zare, "Angular distribution of photoelectrons", J. Chem. Phys., 48 (2), 942, 1968.

[33] J. H. D. Eland, "The photoelectron spectra of isocyanic acid and related compounds", Philos. Trans. R. Soc. London, Ser. A, 268 (1184), 87, 1970.

[34] A. D. Buckingham, B. J. Orr, and J. M. Sichel, "Angular distribution and intensity in molecular photoelectron spectroscopy I. General theory for diatomic molecules", Philos. Trans. R. Soc. London, Ser. A, 268 (1184), 147, 1970.

[35] J. Berkowitz and H. Ehrhardt, "Angular distribution of valence-shell photoelectrons", Phys. Lett., 21 (5), 531, 1966.

[36] J. Berkowitz, H. Ehrhardt, and T. Tekaat, "Spektren und Winkelverteilungen der Photoelektronen von Atomen und Molekülen", Z. Physik, 200 (1), 69, 1967.

[37] C. M. Truesdale, S. Southworth, P. H. Kobrin, D. W. Lindle, G. Thornton, and D. A. Shirley, "Photoelectron angular distributions of $\mathrm{H}_{2} \mathrm{O}$ ", J. Chem. Phys., 76 (2), 860, 1982.

[38] B. Brehm, "Angular distribution of low energy photoelectrons with defined spin orientation", Z. Phys. A: Hadrons Nucl., 242 (3), 195, 1971.

[39] M. S. Banna, B. H. McQuaide, R. Malutzki, and V. Schmidt, "The photoelectron spectrum of water in the $30-140 \mathrm{eV}$ photon energy range", J. Chem. Phys., 84 (9), 4739, 1986. 
[40] D. A. Vroom, A. R. Comeaux, and J. W. McGowan, "Angular distribution of photoelectrons emitted from argon by $584 \AA$ and $744,736 \AA$ radiation", Chem. Phys. Lett., 3 (7), 476, 1969.

[41] J. M. Combes, P. Duclos, M. Klein, and R. Seiler, "The shape resonance", Commun. Math. Phys., 110 (2), 215, 1987.

[42] K. Kondo, N. Sarukura, K. Sajiki, and S.Watanabe, "High order harmonic generation by ultrashort KrF and Ti:Sapphire lasers", Phys. Rev. A, 47 , R2480, 1993.

[43] J. L. Krause, K. J. Schafer, and K. C. Kulander, "High-order harmonic generation from atoms and ions in the high intensity regime", Phys. Rev. Lett., 68, 3535, 1992.

[44] P. M. Paul, E. S. Toma, P. Breger, G. Mullot, F. Auge, P. Balcou, H. G. Muller, and P. Agostini, "Observation of a train of attosecond pulses from high harmonic generation", Science, 292, 1689, 2001.

[45] M. Ferray, A. L'Huillier, X. F. Li, L. A. Lompre, G. Mainfray, and C. Manus, "Multiple-harmonic conversion of $1064 \mathrm{~nm}$ radiation in rare gases", J. Phys. B: At. Mol. Opt. Phys., 21, L31, 1988.

[46] J. Wildenauer, "Generation of the ninth, eleventh, and fifteenth harmonics of iodine laser radiation", J. Appl. Phys., 62 (1), 41, 1987.

[47] J. J. Macklin, J. D. Kmetec, and C. L. G. III, "High-order harmonic generation using intense femtosecond pulses", Phys. Rev. Lett., 70 (6), 766, 1993.

[48] E. A. Gibson, A. Paul, N. Wagner, R. Tobey, S. Backus, I. P. Christov, M. M. Murnane, and H. C. Kapteyn, "High-order harmonic generation up to $250 \mathrm{eV}$ from highly ionized argon", Phys. Rev. Lett., 92 (3), 33001, 2004.

[49] M. Schultze, E. Goulielmakis, M. Uiberacker, M. Hofstetter, J. Kim, D. Kim, F. Krausz, and U. Kleineberg, "Powerful 170-attosecond XUV pulses generated with few-cycle laser pulses and broadband multilayer optics", New J. Phys., 9 (7), 243, 2007. 
[50] P. B. Corkum, "Plasma perspective on strong-field multiphoton ionization", Phys. Rev. Lett., 71, 1994, 1993.

[51] A. P., F. Fabre, G. Mainfray, G. Petite, and N. K. Rahman, "Free-free transitions following six-photon ionization of xenon atoms", Phys. Rev. Lett., $42(17), 1127,1979$.

[52] D. N. Fittinghoff, P. R. Bolton, B. Chang, and K. C. Kulander, "Observation of nonsequential double ionization of helium with optical tunneling", Phys. Rev. Lett., 69, 2642, 1992.

[53] M. Lewenstein, P. Balcou, M. Y. Ivanov, A. L'Huiller, and P. B. Corkum, "Theory of high-harmonic generation by low-frequency laser fields", Phys. Rev. A, 49 (3), 2117, 1994.

[54] J. M. Schins, P. Breger, P. Agostini, R. C. Constantinescu, H. G. Muller, A. Bouhal, G. Grillon, A. Antonetti, and A. Mysyrowicz, "Cross-correlation measurements of femtosecond extreme-ultraviolet high-order harmonics", $J$. Opt. Soc. Am. B, 13 (1), 197, 1996.

[55] A. Bouhal, R. Evans, G. Grillon, A. Mysyrowicz, P. Breger, P. Agostini, R. C. Constantinescu, H. G. Muller, and D. von der Linde, "Cross-correlation measurement of femtosecond noncollinear high-order harmonics", J. Opt. Soc. Am. B, 14 (4), 950, 1997.

[56] T. E. Glover, R. W. Schoenlein, A. H. Chin, and C. V. Shank, "Observation of laser assisted photoelectric effect and femtosecond high order harmonic radiation", Phys. Rev. Lett., 76 (14), 2468, 1996.

[57] J. G. Eden, "High-order harmonic generation and other intense optical field-matter interactions: Review of recent experimental and theoretical advances", Prog. Quantum Electron., 28, 197, 2004.

[58] P. Antoine, B. Carrè, A. L'Huillier, and M. Lewenstein, "Polarization of high-order harmonics", Phys. Rev. A, 55 (2), 1314, 1997. 
[59] R. L. Carman, C. K. Rhodes, and R. F. Benjamin, "Observation of harmonics in the visible and ultraviolet created in $\mathrm{CO}_{2}$-laser-produced plasmas", Phys. Rev. A, 24 (5), 2649, 1981.

[60] K. Budil, P. Salières, A. L'Huillier, T. Ditmire, and M. Perry, "Influence of ellipticity on harmonic generation", Phys. Rev. A, 48 (5), 3437, 1993.

[61] G. P. Zhang, "High harmonic generation in atoms, molecules and nanostructures", Int. J. Mod. Phys. B, 21 (31), 5167, 2007.

[62] S. Kim, J. Jin, Y. Kim, I. Park, Y. Kim, and S. Kim, "High-harmonic generation by resonant plasmon field enhancement", Nature, 453, 757, 2008.

[63] E. Jin and X. Xu, "Enhanced optical near field from a bowtie aperture", Appl. Phys. Lett., 88, 153110, 2006.

[64] T.-D. Onuta, M. Waegele, C. DuFort, W. Schaich, and B. Dragnea, "Optical field enhancement at cusps between adjacent nanoapertures", Nano Lett., \%, 557, 2007.

[65] W.-X. Sun and Z.-X. Shen, "Optimizing the near field around silver tips", $J$. Opt. Soc. Am. A, 20, 2254, 2003.

[66] Y.-F. T. et al., "Fabrication of an ultra-nanocrystalline diamond-coated silicon wire array with enhanced field-emission performance", Nanotechnology, 18, 435703, 2007.

[67] K. R. Siefermann, Y. Liu, E. Lugovoy, O. Link, M. Faubel, U. Buck, B. Winter, and B. Abel, "Binding energies, lifetimes and implications of bulk and interface solvated electrons in water", Nat. Chem., 2 (4), 274, 2010.

[68] O. Link, Femtosekunden- Photoelektronenspektroskopie mit extrem ultravioletter Strahlung an Flüssigkeitsgrenzflächen, Ph.D. thesis, Universität Göttingen, 2007.

[69] R. v. Benten, Der Einfluß von chemischer Konstitution, Symmetrie und molekularer Umgebung auf die intramolekulare Schwingungsrelaxation aromatischer Moleküle, Ph.D. thesis, Universität Göttingen, 2006. 
[70] J. O. Hirschfelde, C. F. Curtiss, and R. B. Bird, Molecular theory of gases and liquids, Wiley, 1954.

[71] M. Faubel and T. Kisters, "Non-equilibrium molecular evaporation of carboxylic acid dimers", Nature, 339, 527, 1989.

[72] M. Faubel, personal communication, 2009.

[73] M. Michaud, A. Wen, and L. Sanche, "Cross sections for low-energy (1-100 $\mathrm{eV}$ ) electron elastic and inelastic scattering in amorphous ice", Radiat. Res., 159, 3, 2003.

[74] N. Ottosson, M. Faubel, S. E. Bradforth, P. Jungwirth, and B. Winter, "Photoelectron spectroscopy of liquid water and aqueous solution: Electron effective attenuation lengths and emission-angle anisotropy", J. Electron. Spectrosc. Relat. Phenom., 177 (2-3), 60, 2010.

[75] B. Winter and M. Faubel, "Photoemission from liquid aqueous solutions", Chem. Rev., 106, 1176, 2006.

[76] O. F. Hagena, "Nucleation and growth of clusters in expanding nozzle flows", Surf. Sci., 106, 101, 1981.

[77] O. F. Hagena, "Condensation in free jets: Comparison of rare gases and metals", Z. Phys. D, 4, 291, 1987.

[78] C. Bobbert, S. Schütte, C. Steinbach, and U. Buck, "Fragmentation and reliable size distribution of large amonia and water clusters", Eur. Phys. J. D, 19, 183, 2002.

[79] J. B. Hasted, "Liquid water: Dielectric properties", in F. Franks (editor), Water - A Comprehensive Treatise, vol. 1, chap. 7, pp. 205-309, Plenum Press, New York, 1972.

[80] S. A. Clough, Y. Beers, G. P. Klein, and L. S. Rothman, "Dipole moment of water from Stark measurements of $\mathrm{H}_{2} \mathrm{O}, \mathrm{HDO}$, and $\mathrm{D}_{2} \mathrm{O}$ ", J. Chem. Phys., 59, 2254, 1973. 
[81] M. W. Schmidt, K. K. Baldridge, J. A. Boatz, S. T. Elbert, M. S. Gordon, J. H. Jensen, S. Koseki, N. Matsunaga, K. A. Nguyen, S. Su, T. L. Windus, M. Dupuis, and J. A. Montgomery, "General atomic and molecular electronic structure system", J. Comput. Chem., 14 (11), 1347, 1993.

[82] A. K. Soper, F. Bruni, and M. A. Ricci, "Site-site pair correlation functions of water from 25 to $400^{\circ} \mathrm{C}$ : Revised analysis of new and old diffraction data", J. Chem. Phys., 106 (1), 247, 1997.

[83] F. H. Stillinger, "Water revisited", Science, 209 (4455), 451, 1980.

[84] S. J. Suresh and V. M. Naik, "Hydrogen bond thermodynamic properties of water from dielectric constant data", J. Chem. Phys., 113 (21), 9727, 2000.

[85] C.-M. Liegener and R. Chen, "Calculation of the Auger spectrum of liquid water", J. Chem. Phys., 88, 2618, 1988.

[86] P. Hunt, M. Sprik, and R. Vuilleumier, "Thermal versus electronic broadening in the density of states of liquid water", Chem. Phys. Lett., 376, 68, 2003.

[87] D. Prendergast, J. C. Grossman, and G. Galli, "The electronic structure of liquid water within density-functional theory", J. Chem. Phys., 123 (1), 14501, 2005.

[88] S. Maw, H. Sato, S. Ten-no, and F. Hirata, "Ab initio study of water: Selfconsistent determination of electronic structure and liquid state properties", Chem. Phys. Lett., 276, 20, 1997.

[89] G. Öhrwall, R. F. Fink, M. Tchaplyguine, L. Ojamäe, M. Lundwall, R. R. T. Marinho, A. N. de Brito, S. L. Sorensen, M. Gisselbrecht, R. Feifel, T. Rander, A. Lindblad, J. Schuz, L. J. Sæthre, N. Mårtenssen, S. Svensson, and O. Björneholm, "The electronic structure of free water clusters probed by Auger electron spectroscopy", J. Chem. Phys., 123, 054310, 2005.

[90] I. B. Müller and L. S. Cederbaum, "Ionization and double ionization of small water clusters", J. Chem. Phys., 125, 204305, 2006. 
[91] J. K. Gregory, D. C. Clary, K. Liu, M. G. Brown, and R. J. Saykally, "The water dipole moment in water clusters", Science, 2\%4, 814, 1997.

[92] P. C. do Couto, B. J. C. Cabral, and S. Canuto, "Electron binding energies of water clusters: Implications for the electronic properties of liquid water", Chem. Phys. Lett., 429, 129, 2006.

[93] S. S. Xantheas, "Cooperativity and hydrogen bonding network in water clusters", Chem. Phys., 258, 225, 2000.

[94] H. S. Frank and W. Wen, "Ion-solvent interaction. Structural aspects of ion-solvent interaction in aqueous solutions: a suggested picture of water structure", Discuss. Faraday Soc., 24, 133, 1957.

[95] K. Y. Yu, J. C. McMenamin, and W. E. Spicer, "UPS measurements of molecular energy level of condensed gases", Surf. Sci., 50, 149, 1975.

[96] A. E. Reed, L. A. Curtiss, and F. Weinhold, "Intermolecular interactions from a natural bond orbital, donor-acceptor viewpoint", Chem. Rev., 88 (6), 899, 1988.

[97] U. Gelius, E. Basilier, S. Svensson, T. Bergmark, and K. Siegbahn, "A high resolution ESCA instrument with X-ray monochromator for gases and solids", J. Electron. Spectrosc. Relat. Phenom., 2, 405, 1974.

[98] A. D. Buckingham, P. W. Fowler, and J. M. Hutson, "Theoretical studies of van der Waals molecules and intermolecular forces", Chem. Rev., 88 (6), 963, 1988.

[99] K. Morokuma, "Why do molecules interact? The origin of electron donoracceptor complexes, hydrogen bonding and proton affinity", Acc. Chem. Res., 10 (8), 294, 1977.

[100] S. Iuchi, S. Izvekov, and G. A. Voth, "Are many-body electronic polarization effects important in liquid water?", J. Chem. Phys., 126 (12), 124505, 2007. 
[101] L. X. Dang, "Importance of polarization effects in modeling the hydrogen bond in water using classical molecular dynamics techniques", J. Phys. Chem. B, 102 (3), 620, 1998.

[102] B. Guillot, "A reappraisal of what we have learnt during three decades of computer simulations on water", J. Mol. Liq., 101 (1-3), 219, 2002.

[103] E. E. Dahlke and D. G. Truhlar, "Assessment of the pairwise additive approximation and evaluation of many-body terms for water clusters", J. Phys. Chem. B, 110 (22), 10595, 2006.

[104] U. Buck, personal communication, 2010.

[105] G. Öhrwall, M. Tchaplyguine, M. Gisselbrecht, M. Lundwall, R. Feifel, T. Rander, J. Schulz, R. R. T. Marinho, A. Lindgren, S. L. Sorensen, S. Svensson, and O. Björneholm, "Observation of elastic scattering effects on photoelectron angular distributions in free Xe clusters", J. Phys. B: At. Mol. Opt. Phys., 36 (19), 3937, 2003.

[106] M. Roche and D. Salhub, "Scattered-wave calculations of photoionization cross-section and asymmetry parameter for $\mathrm{CO}, \mathrm{H}_{2} \mathrm{O}$ and $\mathrm{H}_{2} \mathrm{~S}$ ", J. Electron. Spectrosc. Relat. Phenom., 19, 273, 1980.

[107] P. Sivestrelli and M. Parrinello, "Structural, electronic, and bonding properties of liquid water from first principles", J. Chem. Phys., 111 (8), 3572, 1999.

[108] G. P. Parravicini and L. Resca, "Electronic states and optical properties in cubic ice", Phys. Rev. B, 8 (6), 3009, 1973.

[109] P. Wernet, D. Nordlund, U. Bergmann, M. Cavalleri, M. Odelius, H. Ogasawara, L. A. Näslund, T. K. Hirsch, L. Ojamäe, P. Glatzel, L. G. M. Pettersson, and A. Nilsson, "The structure of the first coordination shell in liquid water", Science, 304, 995, 2004.

[110] A. K. Soper, "Orientational correlation function for molecular liquids: The case of liquid water", J. Chem. Phys., 101 (8), 6888, 1994. 
[111] A. K. Soper, "An asymmetric model for water structure", J. Phys.: Condens. Matter, 17 (45), 3273, 2005.

[112] H.-S. Lee and M. E. Tuckerman, "Structure of liquid water at ambient temperature from ab initio molecular dynamics performed in the complete basis set limit", J. Chem. Phys., 125 (15), 154507, 2006.

[113] S. B. C. Lehmann, C. Spickermann, and B. Kirchner, "Quantum cluster equilibrium theory applied in hydrogen bond number studies of water. 2 . Icebergs in a two-dimensional water continuum?", J. Chem. Theory Comput., 5 (6), 1650, 2009.

[114] W. R. Cannon and S. J. Benkovic, "Solvation, reorganization energy, and biological catalysis", J. Biol. Chem., 273 (41), 26257, 1998.

[115] G. A. Olah, A. Goeppert, and G. K. S. Prakash, Beyond Oil and Gas: The Methanol Economy, Wiley-VCH, Weinheim, 1st ed., 2006.

[116] F. J. Lovas and H. Hartwig, "The microwave spectrum of the methanol dimer for K = 0 and 1 states", J. Mol. Spectrosc., 185 (1), 98, 1997.

[117] S. Kashtanov, A. Augustson, J.-E. Rubensson, J. Nordgren, H. Ågren, J.H. Guo, and Y. Luo, "Chemical and electronic structures of liquid methanol from X-ray emission spectroscopy and density functional theory", Phys. Rev. B, 71 (10), 104205, 2005.

[118] B. H. Torrie, S. X. Weng, and B. M. Powell, "Structure of the $\alpha$-phase of solid methanol", Mol. Phys., 67 (3), 575, 1989.

[119] M. Haughney, M. Ferrario, and I. R. McDonald, "Molecular-dynamics simulation of liquid methanol", J. Phys. Chem., 91 (19), 4934, 1987.

[120] E. Tsuchida, Y. Kanada, and M. Tsukada, "Density-functional study of liquid methanol", Chem. Phys. Lett., 311 (3-4), 236, 1999.

[121] T. Yamaguchi, K. Hidaka, and A. K. Soper, "The structure of liquid methanol revisited: A neutron diffraction experiment at $-80^{\circ} \mathrm{C}$ and $+25^{\circ} \mathrm{C} "$, Mol. Phys., 97 (4), 603, 1999. 
[122] A. K. Adya, L. Bianchi, and C. J. Wormald, "The structure of liquid methanol by H/D substitution technique of neutron diffraction", J. Chem. Phys., 112 (9), 4231, 2000.

[123] D. G. Montague, I. P. Gibson, and J. C. Dore, "Structural studies of liquid alcohols by neutron diffraction - I. Deuterated methyl alcohol $\mathrm{CD}_{3} \mathrm{OD}$ ", Mol. Phys., 44 (6), 1355, 1981.

[124] M. Magini, G. Paschina, and G. Piccaluga, "On the structure of methyl alcohol at room temperature", J. Chem. Phys., 77 (4), 2051, 1982.

[125] L. Pauling, The Nature of the Chemical Bond, Cornell University Press, Ithaca, NY, USA, 3rd ed., 1960.

[126] U. Buck, B. Schmidt, and J. G. Siebers, "Structural transitions and thermally averaged infrared spectra of small methanol clusters", J. Chem. Phys., 99 (12), 9428, 1993.

[127] M. Abu-samha, K. J. Børve, L. J. Sæthre, G. Öhrwall, H. Bergersen, T. Rander, O. Björneholm, and M. Tchaplyguine, "Lineshapes in carbon 1s photoelectron spectra of methanol clusters", Phys. Chem. Chem. Phys., 8 (21), 2473, 2006.

[128] U. Buck, J.-G. Siebers, and R. J. Wheatley, "Structure and vibrational spectra of methanol clusters from a new potential model", J. Chem. Phys., 108 (1), 20, 1998.

[129] R. A. Provencal, J. B. Paul, K. Roth, C. Chapo, R. N. Casaes, R. J. Saykally, G. S. Tschumper, and H. F. Shaefer III, "Infrared cavity ringdown spectroscopy of methanol clusters: Single donor hydrogen bonding", J. Chem. Phys., 110 (9), 4258, 1999.

[130] H. Bergersen, M. Abu-samha, A. Lindblad, R. R. T. Marinho, G. Öhrwall, M. Tchaplyguine, K. J. Børve, S. Svensson, and O. Björneholm, "Two size regimes of methanol clusters produced by adiabatic expansion", J. Chem. Phys., 125 (18), 184303, 2006. 